TRANSACTIONS OF THE

AMERICAN MATHEMATICAL SOCIETY

Volume 350, Number 6, June 1998, Pages 2141-2183

S $0002-9947(98) 01990-4$

\title{
EQUIVARIANT NOVIKOV CONJECTURE FOR GROUPS ACTING ON EUCLIDEAN BUILDINGS
}

\author{
DONGGENG GONG
}

\begin{abstract}
We prove the equivariant Novikov conjecture for groups acting on Euclidean buildings by using an equivariant Hilsum-Skandalis method. We also obtain an equivariant version of the Connes-Gromov-Moscovici theorem for almost flat $C^{*}$-algebra bundles.
\end{abstract}

\section{INTRODUCTION}

The well-known Novikov conjecture states that the higher signature of a closed oriented Riemannian manifold is a homotopy invariant. There are many versions of this conjecture, and a lot of work has been devoted to them (cf. [BCH] [BHM] [Cap] $[\mathrm{CaP}][\mathrm{CoJ}][\mathrm{CoM}][\mathrm{CGM}][\mathrm{FaJ}][\mathrm{FeW}]$ [Gong5] [Kas] [KaS] [Lus] [Mis1], [Mis2] [Roe] [Ros] [RoW2] [Wein]). In particular, we mention an equivariant Novikov conjecture proposed by Rosenberg and Weinberger [RoW2] which says that for a commutative diagram

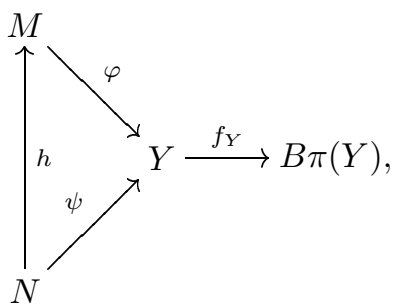

of closed oriented Riemannian $G$-manifolds $M$ and $N$, a $G$-space $Y$ with $G$-maps $\varphi$ and $\psi$, and a $G$-pseudo-equivalence $h$, the equivariant signature elements $\left[D_{M}\right]$ and $\left[D_{N}\right]$ of $M$ and $N$ satisfy $\left(f_{Y}\right)_{*} \varphi_{*}\left(\left[D_{M}\right]\right)=\left(f_{Y}\right)_{*} \psi_{*}\left(\left[D_{N}\right]\right)$ in $K_{*}^{G}(B \pi(Y))$, provided the equivariant $K$-homology $K_{*}^{G}(B \pi(Y))$ of $B \pi(Y)$ is finitely generated over the representation ring $R(G)$ of the compact Lie group $G$, where the actions of $G$ on $M$ and $N$ are given by isometries and $B \pi(Y)$ is the classifying space of the fundamental groupoid $\pi(Y)$ of $Y$. We refer to Section 5 for a precise definition. Rosenberg and Weinberger proved this conjecture for $Y$ a complete Riemannian $G$-manifold of nonpositive curvature with the finite generation condition on $K_{*}^{G}(B \pi(Y))$ [RoW2]. One goal of this paper is to verify this equivariant Novikov conjecture for groups acting on Euclidean buildings without the condition of finite

Received by the editors June 27, 1994 and, in revised form, August 9, 1996.

1991 Mathematics Subject Classification. Primary 46L80; Secondary 55N15, 19K56, 58G12.

Key words and phrases. Equivariant Novikov conjecture, equivariant signature elements, $C^{*}$ algebra bundles, Euclidean buildings.

Supported in part by the NSF. 
generation of $K_{*}^{G}(B \pi(Y))$. This extends the Rosenberg-Weinberger theorem by removing the finite generation of $K_{*}^{G}(B \pi(Y))$ and by allowing $Y$ to be a more general $G$-space.

Our method of proving the above mentioned result is different from that of [RoW2]. We planned originally to use equivariant cyclic cohomology to consider the equivariant Novikov conjecture. For this reason, we have rather systematically developed equivariant cyclic cohomology and its application to equivariant index theory [Gong1]-[Gong4]. However, due to the lack of an equivariant version of Connes-Moscovici's $\Gamma$-index theorem [CoM] and of equivariant Mishchenko symmetric signature for a general compact Lie group (these are still open problems), we were forced to seek another approach. We then realized that the Hilsum-Skandalis method [HiS] can be adapted to the equivariant situation to overcome the above problems without assuming the finite generation of $K_{*}^{G}(B \pi(Y))$. Essentially, the equivariant Hilsum-Skandalis method is to compare directly two equivariant signature elements in an equivariant $K$-theory group. This enables us to avoid the problem of the equivariant Mishchenko symmetric signature. Furthermore, we are able to deal with much more general equivariant $C^{*}$-algebra bundles, to which the applicability of equivariant cyclic cohomology is still not clear. As a by-product, we also obtain an equivariant Connes- Gromov-Moscovici theorem which is interesting on its own, and which is the second goal of the present paper.

The main theorems of the paper are stated as follows.

Theorem 1. Let $M$ and $N$ be two closed oriented Riemannian $G$-manifolds of even dimensions. Suppose that $h: N \rightarrow M$ is an orientation preserving homotopy equivalence which is equivariant. Then for every unitary flat $G$-A-bundle $E$ over $M$, the equivariant analytic signatures of $M$ and $N$ with coefficients in $E$ and $h^{*}(E)$, resp., are equal:

$$
G-\operatorname{Sig}\left(D_{E}\right)=G-\operatorname{Sig}\left(D_{h^{*}(E)}\right) \in K_{0}^{G}(\mathcal{A}) .
$$

In other words, the equivariant signature of $M$ with coefficients in a unitary flat $G$ - $\mathcal{A}$-bundle is a homotopy invariant in the sense of Theorem 1 . Here $K_{0}^{G}(\mathcal{A})$ is the equivariant $K$-theory group of a $G$ - $C^{*}$-algebra $\mathcal{A}$. By a $G$ - $\mathcal{A}$-bundle $E$ over $M$, we mean that $E$ is a bundle over $M$ with fibers modeled on a finitely generated projective Hilbert $G$-A-module $E_{0}$. The following equivariant Connes-GromovMoscovici theorem extends Theorem 1 to the case of almost flat $G$ - $\mathcal{A}$-bundles.

Theorem 2. Let $M, N, h$ and $G$ be as in Theorem 1. Then there exists a constant $\tau>0$ such that for every $\tau$-flat $G$-A-bundle $E$ over $M$,

$$
G-\operatorname{Sig}\left(D_{E}\right)=G-\operatorname{Sig}\left(D_{h^{*}(E)}\right) \in K_{0}^{G}(\mathcal{A}) .
$$

Here we say $E$ is $\tau$-flat if there exists a connection $\nabla^{E}$ on $E$ such that the norm of its curvature is less than or equal to $\tau$ (cf. Section 4 ). We will use Theorem 1 as a crucial step to prove the following equivariant Novikov conjecture.

Theorem 3. Let $X$ be a locally finite Euclidean building and $B$ be its geometric realization. Assume $Y=B$ in (1). Suppose $G$ acts on $Y$ by type permuting isometries. Then $\varphi_{*}\left(\left[D_{M}\right]\right)=\psi_{*}\left(\left[D_{N}\right]\right)$.

We refer to Section 5 for the terminology in Theorem 3. Another question studied in this paper is the existence of the equivariant maps in (1). We have the following theorem on the existence of an equivariant map from $\tilde{M}$ to a metric space $W$ of nonpositive curvature, where $\tilde{M}$ is the universal covering space of $M$. 
Theorem 4. Let $M$ be a closed oriented Riemannian G-manifold, $W$ a (simply connected) metric space of nonpositive curvature, and $\Gamma$ the closed subgroup of the diffeomorphism group Diff $(\tilde{M})$ of $\tilde{M}$ which covers the $G$-action on $M$. Suppose that $\rho: \Gamma \rightarrow \operatorname{Isom}(W)$ is a continuous homomorphism and $\Gamma$ acts on $W$ via $\rho$. Then there exists a $\Gamma$-equivariant map from $\tilde{M}$ to $W$.

Note that $\Gamma$ is an extension of the fundamental group $\pi_{1}(M)$ of $M$ by $G$. Theorem 4 extends the Korevaar-Schoen theorem $[\mathrm{KoS}]$ on the existence of a $\pi_{1}(M)$ equivariant map from $\tilde{M}$ to $W$ which is fundamental in the super rigidity [GrS]. Consequently, we obtain a general result on the existence of the equivariant maps in (1). We point out that the metric spaces of nonpositive curvature include the geometric realization $B$ of the Euclidean building $X$ and complete simply connected Riemannian manifolds of nonpositive curvature.

The paper is arranged as follows. In Section 2 we will define the so-called equivariant signature-type elements in the equivariant operator $K$-theory group which are modeled on the signature operator[AtS]. We will then obtain the main machinery of this paper: that is, to give sufficient conditions for two equivariant signaturetype elements to be equal. In Sections 3 and 4 we will verify these sufficient conditions for the signature elements with coefficients in flat and almost flat equivariant $C^{*}$-algebra bundles, respectively. Hence, we get the equivariant Connes-GromovMoscovici theorem. We should point out that the method here will be used in $[\mathrm{GoR}]$ to prove the homotopy invariance of the family Novikov-Shubin invariants. We also expect that the Hilsum-Skandalis method could be used for a $\mathbb{Z} / k$-version of the Novikov conjecture (for $\mathbb{Z} / k$-manifolds), which will be discussed in another paper. The result in Section 3 for the flat case will provide us a substitute for the equivariant Mishchenko symmetric signature. This observation is an essential point of our approach to the equivariant Novikov conjecture. We will use this result together with injectivity of the assembly operator $K$-theory map $\beta$ in Section 5 to prove Theorem 3 and its extended version, namely, for those $Y$ in (1) having Euclidean buildings as the universal covering spaces. Our argument also works for $Y$ a complete Riemannian $G$-manifold of nonpositive curvature. Therefore we obtain the Rosenberg-Weinberger theorem without the finite generation of $K_{*}^{G}(B \pi(Y))$. Finally, we will prove Theorem 4 in Section 6.

This paper is based on Chapters 9 and 10 of [Gong3]. I would like to thank the following people for their helpful communications, discussions and preprints: W.C. Hsiang, Lowell Jones, Erik Pedersen, Jonathan Rosenberg, Nicolae Teleman, and Shmuel Weinberger. My special thanks go to Ran Douglas, Blaine Lawson, Marie-Louis Michelsohn, Joel Pincus and Mel Rothenberg for their important encouragement and advice. I am also very grateful to Richard Schoen for his advice on Section 6, and to the referee for helpful comments.

\section{Signature-Type Elements in Equivariant $K$-Theory}

This section is largely a generalized version of $[\mathrm{HiS}]$ and $[\mathrm{KaM}]$ to the equivariant case. Our effort here is to deal with some technical points about group actions.

To begin with, we assume throughout this section that $G$ is a compact group, $\mathcal{A}$ is a $G$ - $C^{*}$-algebra over $\mathbb{C}$ and $\mathcal{E}$ is a right $G$ - $\mathcal{A}$-module. Here $G$ acts on $\mathcal{A}$ and $\mathcal{E}$ by continuous automorphisms which are compatible with the module structure, 
i.e., $g(x a)=g(x) g(a), g \in G, x \in \mathcal{E}, a \in \mathcal{A}$. If $\mathcal{E}$ is graded with a grading operator $\varepsilon\left(\varepsilon^{2}=1\right)$, then we require $g \varepsilon=\varepsilon g, \forall g \in G$. See [Bla] for the terminology.

Definition 1. Suppose that $Q$ is a $\mathbb{C}$-sesquilinear map from $\mathcal{E} \times \mathcal{E}$ to $\mathcal{A}$.

(i) If $Q(\xi, \eta)=Q(\eta, \xi)^{*}, Q(\xi, \eta a)=Q(\xi, \eta) a$ and $g Q(\xi, \eta)=Q(g \xi, g \eta)$ for $a \in$ $\mathcal{A}, \xi, \eta \in \mathcal{E}, g \in G$, then $Q$ is called an equivariant quadratic form on $\mathcal{E}$.

(ii) If $\mathcal{E}$ is graded and $Q(\xi, \eta)=0$ for $\partial \xi=\partial \eta$, then $Q$ is of degree 1 .

(iii) $Q$ is regular if there is an equivariant $\mathcal{A}$-linear bijection $T$ on $\mathcal{E}, g T=T g$, such that $\mathcal{E}$ with the scalar product $\langle$.$\rangle defined by \langle\xi, \eta\rangle=Q(\xi, T \eta)$ is a Hilbert $G$ - $\mathcal{A}$-module. $T$ is then said to be compatible with $Q$.

(iv) A scalar product $\langle\rangle:. \mathcal{E} \times \mathcal{E} \rightarrow \mathcal{A}$ is compatible with $Q$ if $\langle\xi, \eta\rangle=Q(\xi, T \eta)$ for some $T$ compatible with $Q$.

(v) Two Hilbert $G$ - $\mathcal{A}$-scalar products $\langle.\rangle_{1}$ and $\langle.\rangle_{2}$ on $\mathcal{E}$ are compatible if there exists an equivariant $\mathcal{A}$-linear invertible operator $T$ on $\mathcal{E}$ such that $\langle\xi, \eta\rangle_{2}=\langle\xi, T \eta\rangle_{1}$.

Remark 1. (a) Clearly, $T$ in (v) is unique, and any $T$ satisfying $\langle\xi, \eta\rangle_{2}=\langle\xi, T \eta\rangle_{1}$ is injective.

(b) If there are adjoint operators $S_{1}^{*}$ and $S_{2}^{*}$ of an operator $S$ on $\mathcal{E}$ with respect to the compatible scalar products $\langle.\rangle_{1}$ and $\langle.\rangle_{2}$, then $S_{2}=T^{-1} S_{1}^{*} T$. In fact,

$$
\langle S \xi, \eta\rangle_{2}=\langle S \xi, T \eta\rangle_{1}=\left\langle\xi, S_{1}^{*} T \eta\right\rangle_{1}=\left\langle\xi, T^{-1} S_{1}^{*} T \eta\right\rangle_{2}=\left\langle\xi, S_{2}^{*} \eta\right\rangle_{2} .
$$

(c) $T$ in $(v)$ is selfadjoint and positive with respect to $\langle.\rangle_{1}$ and $\langle.\rangle_{2}$ :

$$
\langle T \xi, \eta\rangle_{1}=\overline{\langle\xi, T \eta\rangle_{1}}=\overline{\langle\xi, \eta\rangle_{2}}=\langle\eta, \xi\rangle_{2}=\langle\eta, T \xi\rangle_{1} .
$$

Thus, $T_{1}^{*}=T$ and $T_{2}^{*}=T^{-1} T_{1}^{*} T=T$. Clearly, $0<\langle\xi, \xi\rangle_{2}=\langle\xi, T \xi\rangle_{1}$ and $\langle\xi, T \xi\rangle_{2}=\langle T \xi, T \xi\rangle_{1}>0$ for $\xi \neq 0$ in $\mathcal{E}$. Hence $T$ is positive.

(d) If two scalar products $\langle.\rangle_{1}$ and $\langle.\rangle_{2}$ on $\mathcal{E}$ are compatible with $Q$, then $\langle.\rangle_{1}$ and $\langle.\rangle_{2}$ are compatible. In fact, let $\langle\xi, \eta\rangle_{i}=Q\left(\xi, T_{i} \eta\right), i=1,2$. Then $\langle\xi, \eta\rangle_{2}=$ $Q\left(\xi, T_{2} \eta\right)=Q\left(\xi, T_{1}\left(T_{1}^{-1} T_{2}\right) \eta\right)=\left\langle\xi, T_{1}^{-1} T_{2} \eta\right\rangle_{1}$.

(e) If $T$ is compatible with $Q$, then $T$ is selfadjoint and there exists an equivariant operator $T_{1}$ on $\mathcal{E}$ compatible with $Q$ such that $T_{1}^{2}=I$. In fact,

$$
\langle T \xi, \eta\rangle=\overline{Q(T \eta, T \xi)}=\overline{\langle T \eta, \xi\rangle}=\langle\xi, T \eta\rangle .
$$

$T$ is selfadoint. Hence $T^{-1}$ is also selfadjoint. Since $T^{2}$ is invertible and positive, $U=\left|T^{2}\right|^{\frac{1}{2}}$ is well defined, invertible and positive. Let $T_{1}=T U^{-1}$. Then $T_{1}^{2}=I$, and $Q\left(\xi, T_{1} \eta\right)=Q\left(\xi, T U^{-1} \eta\right)=\left\langle\xi, U^{-1} \eta\right\rangle$ is a Hilbert scalar product on $\mathcal{E}$. $T_{1}$ is compatible with $Q$.

$(f)$ If $\mathcal{E}$ is endowed with scalar product $\langle$.$\rangle compatible with Q,\langle\xi, \eta\rangle=Q(\xi, T \eta)$, and $S$ is an operator on $\mathcal{E}$ such that there is an adjoint $S^{*}$ with respect to $\langle$.$\rangle , then$ $S^{\prime}=T S^{*} T^{-1}$ is the conjugate of $S$ with respect to $Q$. Indeed,

$$
Q(S \xi, \eta)=\left\langle S \xi, T^{-1} \eta\right\rangle=\left\langle\xi, T^{-1}\left(T S^{*} T^{-1}\right) \eta\right\rangle=Q\left(\xi, T S^{*} T^{-1} \eta\right) .
$$

Let $\mathcal{E}$ be a Hilbert $G$ - $\mathcal{A}$-module with a scalar product $\langle$.$\rangle . Recall that the space$ $\mathcal{L}(\mathcal{E})$ of bounded operators on $\mathcal{E}$ consists of all continuous $\mathcal{A}$-linear maps $S: \mathcal{E} \rightarrow \mathcal{E}$ such that its adjoint $S^{*}$ exists and is $\mathcal{A}$-linear. The space $\mathcal{K}(\mathcal{E})$ of compact operators on $\mathcal{E}$ is the ideal of $\mathcal{L}(\mathcal{E})$ generated by $Q_{x, y}, x, y \in \mathcal{E}$, where $Q_{x, y}(z)=x\langle y, z\rangle . G$ acts on $\mathcal{L}(\mathcal{E})$ by $g(S)(\xi)=g S\left(g^{-1}(\xi)\right)$. Denote by $\mathcal{L}_{G}(\mathcal{E})\left(\right.$ resp., $\left.\mathcal{K}_{G}(\mathcal{E})\right)$ all $G$ continuous operators $S$ in $\mathcal{L}(\mathcal{E})$ (resp., $\mathcal{K}(\mathcal{E})$ ), i.e., $g \rightarrow g(S)$ is norm continuous. Obviously, if $S \in \mathcal{L}(\mathcal{E})$ is equivariant, then $S \in \mathcal{L}_{G}(\mathcal{E})$. A regular operator on $\mathcal{E}$ is a densely defined operator $S$ on $\mathcal{E}$ with densely defined adjoint $S^{*}$ such that $I+S^{*} S$ 
has a dense range in $\mathcal{E} . \mathcal{L}(\mathcal{E}), \mathcal{K}(\mathcal{E})$ (resp., $\left.\mathcal{L}_{G}(\mathcal{E}), \mathcal{K}_{G}(\mathcal{E})\right)$ and regular operators do not depend on compatible Hilbert $G$ - $\mathcal{A}$-module scalar products.

Using the convention that 1-graded and 0 -graded mean graded and trivially graded, we have the following definition:

Definition 2. (1) $\mathcal{L}_{G}^{k}(\mathcal{A})$ is the set of all triples $(\mathcal{E}, Q, D)$, where $\mathcal{E}$ is a $k$-graded $G$ - $\mathcal{A}$-module, $Q$ is a strongly nondegenerate $G$-quadratic form of degree $k, k=0,1$, and $D \in \mathcal{L}_{G}(\mathcal{E})$ is $G$-equivariant such that

(a) $D+D^{\prime} \in \mathcal{K}_{G}(\mathcal{E}) ;(b) D^{2} \in \mathcal{K}_{G}(\mathcal{E}) ;(c)$ there are equivariant $S_{1}$ and $S_{2}$ in $\mathcal{L}_{G}(\mathcal{E})$ such that $S_{1} D+D S_{2}-I \in \mathcal{K}_{G}(\mathcal{E}) ;(d) D$ is of degree $k$.

(2) $\mathcal{L}_{G, u}^{k}(\mathcal{A})$ is the set of all triples $(\mathcal{E}, Q, D)$, where $\mathcal{E}$ and $\mathcal{A}$ are the same as in (1), but $D$ is an equivariant regular operator such that

(a) $D+D^{\prime} \in \mathcal{L}_{G}(\mathcal{E}) ;(b) i m(D) \subset \operatorname{dom}(D)$ and $D^{2} \in \mathcal{L}_{G}(\mathcal{E}) ;(c)$ there are equivariant $S_{1}$ and $S_{2}$ in $\mathcal{K}_{G}(\mathcal{E})$ with $\operatorname{im}\left(S_{2}\right) \subset \operatorname{dom}(D), D S_{2}, S_{1} D \in \mathcal{L}_{G}(\mathcal{E})$ and $S_{1} D+D S_{2}-I \in \mathcal{K}_{G}(\mathcal{E}) ;(d) D$ is of degree $k$.

We will see in Section 3 that this definition is modeled on the signature element. The following two elementary lemmas will be used to analyze $\mathcal{L}_{G, u}^{k}(\mathcal{A})$.

Lemma 1 ([HiS]). (a) If $D$ is a densely defined adjointable equivariant operator on a Hilbert $G$-A-module $\mathcal{E}$ and $S \in \mathcal{L}_{G}(\mathcal{E})$ is such that $i m(S) \subset \operatorname{dom}\left(D^{*}\right)$, then $D^{*} S$ is in $\mathcal{L}_{G}(\mathcal{E})$.

(b) If $D$ is a regular adjointable equivariant operator on $\mathcal{E}$ and $S \in \mathcal{L}_{G}(\mathcal{E})$ is such that $S D \in \mathcal{L}_{G}(\mathcal{E})$, then $i m\left(S^{*}\right) \subset \operatorname{dom}\left(D^{*}\right)$ and $D^{*} S^{*}=(S D)^{*}$.

(c) If $D_{1}$ and $D_{2}$ are two regular adjointable equivariant operators on $\mathcal{E}$ such that $D_{1}: \operatorname{dom}\left(D_{1}\right) \rightarrow \mathcal{E}$ is bijective and $\operatorname{dom}\left(D_{1}\right) \subset \operatorname{dom}\left(D_{2}\right)$, then $D_{2} D_{1}^{-1}$ is in $\mathcal{L}_{G}(\mathcal{E})$.

Lemma $2([\mathrm{HiS}])$. Let $D$ be a regular equivariant operator on $\mathcal{E}$ such that $D^{2}=0$. Then $D+D^{*}$ is selfadjoint and regular on $\mathcal{E}$, and $\operatorname{dom}\left(D+D^{*}\right)=\operatorname{dom}(D) \cap$ $\operatorname{dom}\left(D^{*}\right)$.

We now consider the properties of elements in $\mathcal{L}_{G}^{k}(\mathcal{A})$ and $\mathcal{L}_{G, u}^{k}(\mathcal{A})$.

Lemma 3. (a) If $(\mathcal{E}, Q, D) \in \mathcal{L}_{G}^{k}(\mathcal{A})$, then $D+D^{*}$ is invertible modulo $\mathcal{K}_{G}(\mathcal{E})$, where $D^{*}$ is the adjoint of $D$ with respect to the scalar product compatible with $Q$.

(b) If $(\mathcal{E}, Q, D) \in \mathcal{L}_{G, u}^{k}(\mathcal{A})$, then $D+D^{*}$ is regular and selfadjoint with domain equal to $\operatorname{dom}(D) \cap \operatorname{dom}\left(D^{*}\right)$, and has the resolvent in $\mathcal{K}_{G}(\mathcal{E})$.

Proof. We first consider the trivially graded case.

(a) The proof is to find the inverse of $D+D^{*}$ modulo $\mathcal{K}_{G}(\mathcal{E})$. Let $p=S_{1} D, q=$ $D S_{2}$, where $S_{i}$ are as in Definition 2. Since $D^{2} \in \mathcal{K}_{G}(\mathcal{E})$,

$$
p^{2}=S_{1} D S_{1} D=S_{1} D\left(I-D S_{2}+k\right)=S_{1} D+k_{1}=p \quad \bmod \left(\mathcal{K}_{G}(\mathcal{E})\right),
$$

and

$$
q^{2}=D S_{2} D S_{2}=\left(k^{\prime}+I-S_{1} D\right) D S_{2}=D S_{2}+k_{1}^{\prime}=q \quad \bmod \left(\mathcal{K}_{G}(\mathcal{E})\right)
$$

for $k_{i}, k_{i}^{\prime} \in \mathcal{K}_{G}(\mathcal{E})$. Then $q D p=D S_{2} D\left(I-D S_{2}+k\right)=D S_{2} D-k_{2}+D S_{2} D k=$ $\left(I-S_{1} D+k\right) D-k_{2}+D S_{2} D k=D+k_{3}=D \bmod \left(\mathcal{K}_{G}(\mathcal{E})\right)$. Take a projection $\left(\bmod \mathcal{K}_{G}(\mathcal{E})\right) p^{\prime} \in \mathcal{L}_{G}(\mathcal{E})\left[\right.$ Bla] such that $p^{\prime} p=p^{\prime}$ and $p p^{\prime}=p \bmod \left(\mathcal{K}_{G}(\mathcal{E})\right)$. Since 
$p+q-I=S_{1} D+D S_{2}-I \in \mathcal{K}_{G}(\mathcal{E}), p=I-q \bmod \left(\mathcal{K}_{G}(\mathcal{E})\right)$, and $q D p=(I-p) D p=$ $D \bmod \left(\mathcal{K}_{G}(\mathcal{E})\right)$. We get

$$
(I-p) D p=(I-p) D p p^{\prime}=D p^{\prime} \bmod \left(\mathcal{K}_{G}(\mathcal{E})\right)
$$

and

$$
\left(I-p^{\prime}\right)(I-p) D p=\left(I-p^{\prime}\right) D p^{\prime} \bmod \left(\mathcal{K}_{G}(\mathcal{E})\right) .
$$

On the other hand, $\left(I-p^{\prime}\right)(I-p) D p=\left(I-p^{\prime}-p+p^{\prime} p\right) D p=(I-p) D p=D$ $\bmod \left(\mathcal{K}_{G}(\mathcal{E})\right)$. Hence, $D=\left(I-p^{\prime}\right) D p^{\prime} \bmod \left(\mathcal{K}_{G}(\mathcal{E})\right)$. Let $p_{1}=p^{\prime} S_{1}\left(I-p^{\prime}\right), p_{2}=$ $p^{\prime} S_{2}\left(I-p^{\prime}\right)$. We have $p_{1} D=p^{\prime} S_{1} D-p^{\prime} S_{1} p^{\prime} D=p^{\prime} S_{1} D=p^{\prime} p=p^{\prime} \bmod \left(\mathcal{K}_{G}(\mathcal{E})\right)$, since $p^{\prime} D=p^{\prime}\left(I-p^{\prime}\right) D p^{\prime}=0 \bmod \left(\mathcal{K}_{G}(\mathcal{E})\right)$. Then

$$
\begin{aligned}
D p_{2} & =q D p p^{\prime} S_{2}\left(I-p^{\prime}\right)=q D p S_{2}\left(I-p^{\prime}\right)=D S_{2}\left(I-p^{\prime}\right) \\
& =(I-p)\left(I-p^{\prime}\right)=I-p^{\prime} \bmod \left(\mathcal{K}_{G}(\mathcal{E})\right)
\end{aligned}
$$

and

$$
\begin{aligned}
p_{1} & =p^{\prime} S_{1}\left(I-p^{\prime}\right)\left(I-p^{\prime}\right)=p^{\prime} S_{1}\left(I-p^{\prime}\right) D p_{2}=p_{1} D p_{2} \\
& =p^{\prime} p_{2}=p^{\prime} S_{2}\left(I-p^{\prime}\right)=p_{2} \quad \bmod \left(\mathcal{K}_{G}(\mathcal{E})\right)
\end{aligned}
$$

Hence,

$$
\begin{aligned}
\left(p_{1}+p_{1}^{*}\right)\left(D+D^{*}\right) & =p^{\prime}+\left(I-p^{\prime}\right) S_{1}^{*} p^{\prime} D+p_{1} D^{*}+\left(D p_{1}\right)^{*} \\
& =p^{\prime}+\left(D p_{2}\right)^{*}=p^{\prime}+\left(I-p^{\prime}\right)=I \quad \bmod \left(\mathcal{K}_{G}(\mathcal{E})\right) .
\end{aligned}
$$

Here we have used $p^{\prime} D=p^{\prime}\left(I-p^{\prime}\right) D p^{\prime}=0 \bmod \left(\mathcal{K}_{G}(\mathcal{E})\right)$ and $p_{1} D^{*}=p_{2} D^{*}=$ $p^{\prime} S_{2}\left(I-p^{\prime}\right) D^{*}=p^{\prime} S_{2}\left(I-p^{\prime}\right) p^{\prime} D^{*}\left(I-p^{\prime}\right)=0$. This implies that $\left(D+D^{*}\right)^{-1}=p_{1}+p_{1}^{*}$ $\bmod \left(\mathcal{K}_{G}(\mathcal{E})\right)$.

(b) We divide the proof into three steps.

Step 1. First we assume $D^{2}=0$. By Lemma $2, D+D^{*}$ is selfadjoint and regular. Write $S_{1} D+D S_{2}=I-k, S_{i}, k \in \mathcal{K}_{G}(\mathcal{E})$. Since $D^{2}=0,\left(S_{1} D\right)^{2}=S_{1} D\left(I-k-D S_{2}\right)$ $=S_{1} D-S_{1} D k=S_{1} D \bmod \left(\mathcal{K}_{G}(\mathcal{E})\right)$, so we can thus choose $p \in \mathcal{L}_{G}(\mathcal{E})$ such that $p=p^{*}, p^{2}-p \in \mathcal{K}_{G}(\mathcal{E}), p S_{1} D-p \in \mathcal{K}_{G}(\mathcal{E})$ and $S_{1} D p-S_{1} D \in \mathcal{K}_{G}(\mathcal{E})$ [Bla]. Since $I-\left(p S_{1} D+D S_{2}(I-p)\right)=I-D S_{2}-\left(p S_{1} D-D S_{2} p\right)=S_{1} D-\left(p S_{1} D-\left(I-S_{1} D\right) p\right)=$ $S_{1} D(I-p)-p\left(S_{1} D-I\right) \in \mathcal{K}_{G}(\mathcal{E}), D S_{2}(I-p)=I-p S_{1} D=1-p \bmod \left(\mathcal{K}_{G}(\mathcal{E})\right)$ and $p=p S_{1} D=p^{*}=D^{*} S_{1}^{*} p \bmod \left(\mathcal{K}_{G}(\mathcal{E})\right)$. Thus, $q \stackrel{\text { def }}{=} I-D^{*} S_{1}^{*} p-D S_{2}(I-p)=$ $I-\left(p S_{1} D+D S_{2}(I-p)\right) \in \mathcal{K}_{G}(\mathcal{E})$. Note that $\operatorname{dom}\left(D+D^{*}\right)=\operatorname{dom}(D) \cap \operatorname{dom}\left(D^{*}\right)$. By Lemma $1(\mathrm{c})$ and $\operatorname{dom}\left(i+D+D^{*}\right)^{*} \subset \operatorname{dom}(D), \alpha \stackrel{\text { def }}{=} D\left(\left(i+D+D^{*}\right)^{-1}\right)^{*} \in \mathcal{L}_{G}(\mathcal{E})$. This implies that $D=\alpha\left(i+D+D^{*}\right)^{*}$. Also $D^{*}=\beta\left(i+D+D^{*}\right)^{*}$ for some $\beta \in \mathcal{L}_{G}(\mathcal{E})$ by the same argument. Using the fact that $\alpha^{*}=\left(i+D+D^{*}\right)^{-1} D^{*}, \beta^{*}=$ $\left(i+D+D^{*}\right)^{-1} D$, and $S_{1}^{*} p, q$ and $S_{2}(I-p)$ are all in $\mathcal{K}_{G}(\mathcal{E})$, we obtain

$$
\begin{aligned}
\left(i+D+D^{*}\right)^{-1} & =\left(i+D+D^{*}\right)^{-1}\left(q+D^{*} S_{1}^{*} p+D S_{2}(I-p)\right) \\
& =\left(i+D+D^{*}\right)^{-1} q+\left(\alpha^{*} S_{1}^{*} p+\beta^{*} S_{2}(I-p)\right) \in \mathcal{K}_{G}(\mathcal{E}) .
\end{aligned}
$$

This proves that if $D^{2}=0$, then $\left(D+D^{*}\right)$ has resolvent in $\mathcal{K}_{G}(\mathcal{E})$.

Step 2. Suppose $D=D_{0}+a$, where $D_{0}$ is regular, $D_{0}^{2}=0$ and $a \in \mathcal{L}_{G}(\mathcal{E})$. Since $\left\|\left(\lambda i+D_{0}+D_{0}^{*}\right)^{-1}\right\|<\lambda^{-1}$ for sufficiently large $\lambda>0$

$$
\left(\lambda i+D+D^{*}\right)^{-1}=\left(I+\left(\lambda i+D_{0}+D_{0}^{*}\right)^{-1}\left(a+a^{*}\right)\right)^{-1}\left(\lambda i+D_{0}+D_{0}^{*}\right)^{-1} .
$$

This proves that $\left(\lambda i+D+D^{*}\right)^{-1} \in \mathcal{K}_{G}(\mathcal{E})$, since $\left(\lambda i+D_{0}+D_{0}^{*}\right)^{-1} \in \mathcal{K}_{G}(\mathcal{E})$. 
Step 3. In general, we define for $(\mathcal{E}, Q, D) \in \mathcal{L}_{G, u}^{0}(\mathcal{A})$

$$
D_{1}=\left[\begin{array}{cc}
D & D^{2} \\
-I & -D
\end{array}\right], \quad D_{2}=\left[\begin{array}{cc}
D & 0 \\
0 & -D
\end{array}\right] \text {. }
$$

Then $D_{1}^{2}=0, i m\left(D_{1}\right) \subset \operatorname{dom}\left(D_{1}\right)$. Clearly, $\left(\mathcal{E} \oplus \mathcal{E}, Q \oplus(-Q), D_{j}\right) \in \mathcal{L}_{G, u}^{0}(\mathcal{A})$ and $D_{2}=D_{1}-\left[\begin{array}{cc}0 & D^{2} \\ -I & 0\end{array}\right]$. Steps 1 and 2 show that $D_{2}+D_{2}^{*}$ is regular with resolvent in $\mathcal{K}_{G}(\mathcal{E} \oplus \mathcal{E})$. Therefore, $D+D^{*}$ is regular with resolvent in $\mathcal{K}_{G}(\mathcal{E})$.

Now for the graded case we repeat the above proof to get $(a)$ and $(b)$, even though the $D_{i}$ in Step 3 are not of degree 1.

Remark 2. We obtain by the proof of part $(a)$ that if $D \in \mathcal{L}_{G}(\mathcal{E})$ is equivariant and $S_{1}, S_{2} \in \mathcal{L}_{G}(\mathcal{E})$ are such that $D^{2}=0$ and $S_{1} D+D S_{2}=I$, then $D+D^{*}$ is invertible with the inverse $\left(D+D^{*}\right)^{-1}=p^{\prime} S_{1}\left(I-p^{\prime}\right)+\left(I-p^{\prime}\right) S_{1}^{*} p^{\prime}$, where $p^{\prime}$ was given in the proof.

To define the maps from $\mathcal{L}_{G}^{k}(\mathcal{A})$ and $\mathcal{L}_{G, u}^{k}(\mathcal{A})$ to $K_{k}^{G}(\mathcal{A})$, the equivariant $K$ theory group of $\mathcal{A}$, let us recall the definition of Kasparov's $K K$-theory. Let $\mathcal{A}$ and $\mathcal{B}$ be two $G$ - $C^{*}$-algebras. $(\mathcal{E}, \varphi, F)$ is called a Kasparov $G$-bimodule if $\mathcal{E}$ is a countably generated graded Hilbert $G$ - $\mathcal{B}$-module, $\varphi: \mathcal{A} \rightarrow \mathcal{L}(\mathcal{E})$ is an equivariant graded $*$-homomorphism, $F \in \mathcal{L}_{G}(\mathcal{E})$ is of degree 1 such that $[F, \varphi(a)]$, $\left(F-F^{*}\right) \varphi(a),\left(F^{2}-I\right) \varphi(a),(g(F)-F) \varphi(a)$ are in $\mathcal{K}_{G}(\mathcal{E})$ and $\varphi(a) F \in \mathcal{L}_{G}(\mathcal{E})$. Two $G$-bimodules $\left(\mathcal{E}_{j}, \varphi_{j}, F_{j}\right)$ are isomorphic if there exists an equivariant isomorphism $u: \mathcal{E}_{1} \rightarrow \mathcal{E}_{2}$ such that $F_{2}=u F_{1} u^{-1}$. Let $E^{G}(\mathcal{A}, \mathcal{B})$ be the set of all isomorphic classes of $G$-bimodules $(\mathcal{E}, \varphi, F)$.

A homotopy between $\left(\mathcal{E}_{0}, \varphi_{0}, F_{0}\right)$ and $\left(\mathcal{E}_{1}, \varphi_{1}, F_{1}\right)$ is an element $(\mathcal{E}, \varphi, F) \in$ $E^{G}(\mathcal{A}, C([0,1], \mathcal{B}))$ such that $(\mathcal{E}, \varphi, F)$ generates a family $\left\{\left(\mathcal{E}_{t}, \varphi_{t}, F_{t}\right) \in E^{G}(\mathcal{A}, \mathcal{B})\right\}$ given by $\mathcal{E}_{t}=\mathcal{E} \hat{\otimes}_{C([0,1], \mathcal{B})} \mathcal{B}, \varphi_{t}=\left.\varphi\right|_{t}, F_{t}=F \otimes I$. The homotopy gives rise to an equivalence $\sim$. Then the Kasparov $K K$-theory group is $K K^{G}(\mathcal{A}, \mathcal{B})=$ $E^{G}(\mathcal{A}, \mathcal{B}) / \sim$ and $K K_{1}^{G}(\mathcal{A}, \mathcal{B})=K K^{G}\left(\mathcal{A}, \mathcal{B} \otimes C_{2,0}\right) \simeq K K^{G}\left(\mathcal{A} \otimes C_{2,0}, \mathcal{B}\right)$, where $C_{2,0}$ is the Clifford algebra of $\mathbb{C}^{2}$ associated with the quadratic form $Q\left(x_{1}, x_{2}\right)=$ $x_{1}^{2}+x_{2}^{2}$. In particular, $K K_{i}^{G}(\mathbb{C}, \mathcal{B}) \simeq K_{i}^{G}(\mathcal{B}), i=0,1$.

We can also use unbounded modules to define $K K^{G}(\mathcal{A}, \mathcal{B})$ [BaJ] as follows. $(\mathcal{E}, \varphi, F)$ is an unbounded $G$-bimodule if $\mathcal{E}$ is a graded Hilbert $G$ - $\mathcal{B}$-module, $\varphi$ : $\mathcal{A} \rightarrow \mathcal{L}(\mathcal{E})$ is a $G$-*-homomorphism, and $F$ is a selfadjoint regular equivariant operator of degree 1 on $\mathcal{E}$ such that

(a) $\left(I+F^{2}\right)^{-1} \varphi(a) \in \mathcal{K}_{G}(\mathcal{E}), \forall a \in \mathcal{A}$;

(b) $\left\{a \in \mathcal{A}:[F, \varphi(a)]\right.$ is densely defined and extends to an element in $\left.\mathcal{L}_{G}(\mathcal{E})\right\}$ is dense in $\mathcal{A}$.

Let $E_{u}^{G}(\mathcal{A}, \mathcal{B})$ be the set of all unbounded $G$-bimodules. There is a map $\psi$ : $E_{u}^{G}(\mathcal{A}, \mathcal{B}) \rightarrow E^{G}(\mathcal{A}, \mathcal{B})$ given by $\psi((\mathcal{E}, \varphi, F))=\left(\mathcal{E}, \varphi, F\left(I+F^{2}\right)^{-\frac{1}{2}}\right)$.

Suppose that $\mathcal{A}$ and $\mathcal{E}$ are trivially graded. Let $(\mathcal{E}, Q, F) \in \mathcal{L}_{G}^{0}(\mathcal{A})$ and $T \in$ $\mathcal{L}_{G}(\mathcal{E})$ be associated with $Q, T^{2}=I$. Since $T$ is an involution, we can use it to grade $\mathcal{E}$. It follows from $D^{*}=T D^{\prime} T$ that $T\left(D+D^{*}\right)=T D+D^{\prime} T$ and $\left(D+D^{*}\right) T=$ $D T+T D^{\prime}$. Using $D^{\prime}=-D \bmod \left(\mathcal{K}_{G}(\mathcal{E})\right)$, we get $T\left(D+D^{*}\right)=-(D T-T D)=$ $-\left(D+D^{*}\right) T \bmod \left(\mathcal{K}_{G}(\mathcal{E})\right)$. Hence, $D+D^{*}$ is of degree $1 \bmod \left(\mathcal{K}_{G}(\mathcal{E})\right)$. By Lemma $3, D+D^{*}$ is selfadjoint and invertible $\bmod \left(\mathcal{K}_{G}(\mathcal{E})\right)$. Then $D+D^{*}=F\left|D+D^{*}\right|$ $\bmod \left(\mathcal{K}_{G}(\mathcal{E})\right),\left|D+D^{*}\right|=\left(\left(D+D^{*}\right)^{2}\right)^{\frac{1}{2}}$ and $F=\left(D+D^{*}\right)\left|D+D^{*}\right|^{-1} \bmod \left(\mathcal{K}_{G}(\mathcal{E})\right)$. 
Since $D+D^{*}$ is of degree $1 \bmod \left(\mathcal{K}_{G}(\mathcal{E})\right), F$ is of degree $1 \bmod \left(\mathcal{K}_{G}(\mathcal{E})\right)$. We have the following:

(a) $F^{*}=\left|D+D^{*}\right|^{-1}\left(D+D^{*}\right)=F \bmod \left(\mathcal{K}_{G}(\mathcal{E})\right)$;

(b) $F^{2}-I=\left(D+D^{*}\right)^{2}\left|D+D^{*}\right|^{-2}-I=0 \bmod \left(\mathcal{K}_{G}(\mathcal{E})\right)$;

(c) $g(F)-F \in \mathcal{K}_{G}(\mathcal{E})$ : since $g Q(\xi, \eta)=Q(g \xi, g \eta)$ and $g T=T g$, we have $Q\left(g D^{\prime} \xi, g \eta\right)=Q\left(D^{\prime} g \xi, g \eta\right)$. Hence, $g D^{\prime}=D^{\prime} g$, and then $D^{*} g=g D^{*}$, since $D^{*}=T D^{\prime} T$. It follows that $g\left(D+D^{*}\right)=\left(D+D^{*}\right) g$ and $g\left|D+D^{*}\right|=\left|D+D^{*}\right| g$. From this we get $g F=F g \bmod \left(\mathcal{K}_{G}(\mathcal{E})\right)$;

$(d)$ define $\varphi: \mathbb{C} \rightarrow \mathcal{L}_{G}(\mathcal{E})$ by $\varphi(\lambda)=\lambda I . \varphi$ is equivariant.

Therefore, $(\mathcal{E}, \varphi, F)$ is a $G$ - $\mathbb{C}$ - $\mathcal{A}$-module.

Let $(\mathcal{E}, Q, D) \in \mathcal{L}_{G, u}^{0}(\mathcal{A})$, and let $T \in \mathcal{L}_{G}(\mathcal{E})$ be compatible with $Q$ such that $T^{2}=I$. Suppose that the conjugate $D^{\prime}$ of $D$ with respect to $Q$ is $D^{\prime}=-D$. Then $D^{*}=T D^{\prime} T=-T D T$ and $T\left(D+D^{*}\right)=-\left(D+D^{*}\right) T$. This implies that $D+D^{*}$ is of degree 1 if $\mathcal{E}$ is graded by $T$. We know already by Lemma 3 that $D+D^{*}$ is selfadjoint and regular with resolvent in $\mathcal{K}_{G}(\mathcal{E})$. Therefore, $\left(\mathcal{E}, \varphi, D+D^{*}\right)$ is an unbounded $G$ - $\mathbb{C}$ - $\mathcal{A}$-module, where $\varphi(\lambda)=\lambda I$.

Let $\mathcal{A}$ be trivially graded and $(\mathcal{E}, \varphi, D) \in \mathcal{L}_{G}^{1}(\mathcal{A})$ be such that $D^{\prime}=-D$. Then $T\left(D+D^{*}\right)=-\left(D+D^{*}\right) T$, where $T \in \mathcal{L}_{G}(\mathcal{E})$ is compatible with $Q$ and $T^{2}=I$. Let $\varepsilon$ be the grading operator of $\mathcal{E}, \varepsilon^{2}=I$. Since $Q(\xi, \eta)=0$ for $\partial \xi=\partial \eta$, we must have $\varepsilon T=-T \varepsilon$. Using $D \varepsilon=-\varepsilon D$ and $D^{*} \varepsilon=-\varepsilon D^{*}$, we see that $\varepsilon\left(D+D^{*}\right)=-\left(D+D^{*}\right) \varepsilon$. Let $P=\frac{I+i T \varepsilon}{2}, \mathcal{E}_{1}=P \mathcal{E}$ and $D_{1}=P\left(D+D^{*}\right) P$ on $\mathcal{E}_{1}$. Then $P^{2}=P, P^{*}=P, T \varepsilon P=P T \varepsilon$ and $P\left(D+D^{*}\right)=\left(D+D^{*}\right) P$. As for $\mathcal{L}_{G}^{0}(\mathcal{A})$, we can construct $F$ from $\left(\mathcal{E}_{1}, Q, D_{1}\right)$ such that $F^{*}=F, F^{2}=I \bmod \left(\mathcal{K}_{G}(\mathcal{E})\right)$. Then $\left(\mathcal{E}_{1}, F\right)$ determines an element in $K_{1}^{G}(\mathcal{A})$ (cf. [HiS] and [Bla], p.185).

Similarly, we can work out an unbounded $G$ - $\mathbb{C}$ - $\mathcal{A}$-module from $(\mathcal{E}, Q, D) \in$ $\mathcal{L}_{G, u}^{1}(\mathcal{A})$ with $D^{\prime}=-D$ and $\mathcal{A}$ trivially graded, which is denoted by

$$
\left(\mathcal{E}_{1}, Q, P\left(D+D^{*}\right) P\right) .
$$

To summarize, we have the following:

Definition 3. (1) $\psi_{0}: \mathcal{L}_{G}^{0}(\mathcal{A}) \rightarrow K_{0}^{G}(\mathcal{A})$ is given by $\psi_{0}((\mathcal{E}, Q, D))=[(\mathcal{E}, F)]$.

$(2)$ Let $\mathcal{L}_{G, u}^{0^{\prime}}(\mathcal{A})=\left\{(\mathcal{E}, Q, D) \in \mathcal{L}_{G, u}^{0}(\mathcal{A}): D^{\prime}=-D\right\} \cdot \psi_{0, u}: \mathcal{L}_{G, u}^{0^{\prime}}(\mathcal{A}) \rightarrow K_{0}^{G}(\mathcal{A})$ is defined by $\psi_{0, u}((\mathcal{E}, Q, D))=[(\mathcal{E}, F)]$, where $F=\left(D+D^{*}\right)\left(1+\left(D+D^{*}\right)^{2}\right)^{-\frac{1}{2}}$.

(3) Let $\mathcal{A}$ be trivially graded and $\mathcal{L}_{G}^{1^{\prime}}(\mathcal{A})=\left\{(\mathcal{E}, Q, D) \in \mathcal{L}_{G}^{1}(\mathcal{A}): D=-D^{\prime}\right\}$. $\psi_{1}: \mathcal{L}_{G}^{1^{\prime}}(\mathcal{A}) \rightarrow K_{1}^{G}(\mathcal{A})$ is defined by $\psi_{1}((\mathcal{E}, Q, D))=\left[\left(\mathcal{E}_{1}, F\right)\right]$.

(4) Let $\mathcal{A}$ be trivially graded and $\mathcal{L}_{G, u}^{1^{\prime}}(\mathcal{A})=\left\{(\mathcal{E}, Q, D) \in \mathcal{L}_{G, u}^{1}(\mathcal{A}): D=-D^{\prime}\right\}$. $\psi_{1, u}: \mathcal{L}_{G, u}^{1^{\prime}}(\mathcal{A}) \rightarrow K_{1}^{G}(\mathcal{A})$ is defined by $\psi_{1}((\mathcal{E}, Q, D))=\left[\left(\mathcal{E}_{1}, F\right)\right]$, where $F=$ $P\left(D+D^{*}\right) P\left(I+\left(P\left(D+D^{*}\right)\right)^{2}\right)^{-\frac{1}{2}}$.

Remark 3. $\psi_{0}: \mathcal{L}_{G}^{0}(\mathcal{A}) \rightarrow K_{0}^{G}(\mathcal{A})$ is independent of the choice of $F$ and $T$. In fact, if $T_{1}$ and $T_{2}$ are compatible with $Q, T_{i}^{2}=I$. Let $U=T_{2} T_{1} T_{2}$. Then $U^{2}=I$ and $Q(\xi, U \eta)=Q\left(T_{2} \xi, T_{1} T_{2} \eta\right)=\left\langle T_{2} \xi, T_{2} \eta\right\rangle_{1}$. $U$ is compatible with $Q$. By the equivariant stability theorem, we can assume $\mathcal{E}=\mathcal{H}_{\mathcal{A}}$, the universal Hilbert $G$ $\mathcal{A}$-module. Using the invertibility of $U^{\frac{1}{2}}$ and contractibility of $G L\left(\mathcal{H}_{\mathcal{A}}\right)$, we get a homotopy $h_{t}$ in $G L\left(\mathcal{H}_{\mathcal{A}}\right)$ connecting $U^{\frac{1}{2}}$ to $I$. Let $\tilde{h_{t}}=h_{t}^{*} h_{t}$. Then $\tilde{h_{t}}$ is a selfadjoint homotopy between $U$ and $I$. Hence we obtain a homotopy $H_{t}$ connecting $T_{1}$ and $T_{2}$ such that $H_{t}^{2}=I$ and $H_{t}$ is compatible with $Q$. Thus, $\psi_{0}$ is independent of the 
choice of $T$. Clearly, $\psi_{0}$ is independent of the choice of $F$, since two such choices differ by an element in $\mathcal{K}_{G}(\mathcal{E})$.

A similar remark applies to $\psi_{1}$.

The following lemma gives a link between $\mathcal{L}_{G, u}^{0^{\prime}}(\mathcal{A})$ and $\mathcal{L}_{G}^{0}(\mathcal{A})$.

Lemma $4([\mathrm{HiS}])$. Let $(\mathcal{E}, Q, D) \in \mathcal{L}_{G, u}^{0}(\mathcal{A})$ be such that $D^{\prime}=-D$. Let $U=$ $D\left(I+D^{*} D\right)^{-\frac{1}{2}}$. Then, for $\mathcal{E}$ with the scalar product compatible with $Q$,

$$
\psi_{0, u}((\mathcal{E}, Q, D))=\psi_{0}((\mathcal{E}, Q, U)) .
$$

Lemma 5. Let $\mathcal{A}$ be trivially graded. Suppose that $(\mathcal{E}, \varphi, D) \in \mathcal{L}_{G, u}^{1}(\mathcal{A})$ is such that $D^{\prime}=-D$. Let $U=D\left(I+D^{*} D\right)^{-\frac{1}{2}}$. Then, for $\mathcal{E}$ with the scalar product associated with $Q$,

$$
\psi_{1, u}((\mathcal{E}, Q, D))=\psi_{1}((\mathcal{E}, Q, U)) .
$$

Proof. Note that $\psi_{1, u}((\mathcal{E}, Q, D))=\left[\left(\mathcal{E}_{1}, F\right)\right], F=P\left(D+D^{*}\right)\left(I+\left(P\left(D+D^{*}\right)\right)^{2}\right)^{-\frac{1}{2}}$, where $\mathcal{E}_{1}=P \mathcal{E}, P=\frac{I+i T \varepsilon}{2}$, and $\psi_{1}((\mathcal{E}, Q, U))=\left[\left(\mathcal{E}_{1}, F_{1}\right)\right]$ with $F_{1} \in \mathcal{L}_{G}\left(\mathcal{E}_{1}\right)$ such that $P\left(U+U^{*}\right) P-F_{1}\left|P\left(U+U^{*}\right) P\right| \in K_{G}\left(\mathcal{E}_{1}\right)$. The proof is the same as that of Lemma 4.

We now examine when the images under $\psi_{0}$ of two elements in $\mathcal{L}_{G}^{0}(\mathcal{A})$ are equal.

Lemma 6 ([HiS]). Let $(\mathcal{E}, \varphi, D) \in \mathcal{L}_{G}^{0}(\mathcal{A})\left(\right.$ resp., $\left.\mathcal{L}_{G, u}^{0}(\mathcal{A})\right)$.

(a) If $\operatorname{Ker}(D)=i m(D)$ and $D^{\prime}=-D$, then $\psi_{0}((\mathcal{E}, Q, D))=0$.

(b) $\psi_{0}((\mathcal{E},-Q,-D))=-\psi_{0}((\mathcal{E}, Q, D))$.

The same results hold for $\psi_{0, u}$.

Proof. (a) By Lemma 4, it suffices to prove the assertion for the bounded case. By definition of $\psi_{0}$, it is enough to check $F^{2}=I$ and $F^{*}=F$, which are guaranteed by the invertibility of $D+D^{*}$. In fact, if $D+D^{*}$ is invertible, then $\left(\left(D+D^{*}\right)^{2}\right)^{\frac{1}{2}}$ is invertible with the inverse $\left(\left(D+D^{*}\right)^{2}\right)^{-\frac{1}{2}}$ and $F^{2}=\left(\left(D+D^{*}\right)\left(\left(D+D^{*}\right)^{2}\right)^{-\frac{1}{2}}\right)^{2}=I$.

To prove that $D+D^{*}$ is invertible, let us first note that for two Hilbert modules $\mathcal{E}$ and $\mathcal{E}_{1}$ and $S \in \mathcal{L}\left(\mathcal{E}, \mathcal{E}_{1}\right), S S^{*}$ is invertible, provided $S$ is surjective. Indeed, by the open mapping theorem, there is $k>0$ such that $S S^{*} \geq k^{-2}$. We take $\mathcal{E}_{1}=$ $\operatorname{Ker}(D)=i m(D)$ and $S=D: \mathcal{E} \rightarrow i m(D)$. $S$ is surjective. Then $S S^{*}$ is invertible. This implies that zero is at worst an isolated spectral point of $S^{*} S=D^{*} D$, since $\operatorname{Spec}\left(S S^{*}\right) \backslash\{0\}=\operatorname{Spec}\left(S^{*} S\right) \backslash\{0\}$. We can then choose a submodule $\mathcal{E}_{2}$ in $\mathcal{E}$ which is orthogonal to $\mathcal{E}_{1}$ such that $\mathcal{E}=\mathcal{E}_{1} \oplus \mathcal{E}_{2}$ and $D D^{*}$ is invertible on $\mathcal{E}_{1}$ and $D^{*} D$ is invertible on $\mathcal{E}_{2}$. It follows that $D D^{*}+D^{*} D$ is invertible on $\mathcal{E}$. In view of $D^{2}=0$, $\left(D+D^{*}\right)^{2}=D D^{*}+D^{*} D$ is invertible on $\mathcal{E}$. Hence $D+D^{*}$ is invertible in $\mathcal{L}(\mathcal{E})$ and then in $\mathcal{L}_{G}(\mathcal{E})$, since $D+D^{*}$ is equivariant.

(b) If we change $Q$ to $-Q$ and $D$ to $-D$, then $T$ is altered to $-T, D^{*}=$ $T D^{\prime} T$ to $-D^{*}$, and then $F$ to $-F$. Hence, we change $(\mathcal{E}, F)$ to $(\mathcal{E},-F)$, i.e., $\psi_{0}((\mathcal{E},-Q,-D))=[(\mathcal{E},-F)]=-[(\mathcal{E}, F)]=-\psi_{0}((\mathcal{E}, Q, D))$.

Lemma 7. Let $\mathcal{A}$ be trivially graded and $(\mathcal{E}, Q, D) \in \mathcal{L}_{G}^{1}(\mathcal{A})$ (resp., $\left.\mathcal{L}_{G, u}^{1}(\mathcal{A})\right)$.

(a) If $\operatorname{Ker}(D)=i m(D)$ and $D^{\prime}=-D$, then $\psi_{1}((\mathcal{E}, Q, D))=0$.

(b) $\psi_{1}((\mathcal{E},-Q,-D))=-\psi_{1}((\mathcal{E}, Q, D))$, where $-\mathcal{E}$ is equal to $\mathcal{E}$ graded by $-\varepsilon$.

The same results hold for $\psi_{1, u}$. 
Proof. (a) Note that $\left(D+D^{*}\right)$ commutes with $P=\frac{I+i T \varepsilon}{2}$. The proof of Lemma 6 implies that $\left(D+D^{*}\right)$ is invertible in $\mathcal{L}_{G}(\mathcal{E})$. Hence $P\left(D+D^{*}\right)$ is invertible in $\mathcal{L}_{G}\left(\mathcal{E}_{1}\right)$. As a result, $\psi_{1}((\mathcal{E}, Q, D))=0$.

(b) Observe that if $Q$ and $D$ are changed to $-Q$ and $-D$, resp., then $T$ is changed to $-T, \varepsilon$ to $-\varepsilon, D^{*}$ to $-D^{*}, P$ to $P$, and hence $P\left(D+D^{*}\right)$ to $-P\left(D+D^{*}\right)$. The result then follows easily.

\section{Case of Flat Bundles}

We now use the machinery in Section 2 to prove the homotopy invariance of the signature elements with coefficients in flat bundles.

We assume throughout this section that $G$ is a compact Lie group, $\mathcal{A}$ is a $G$ - $C^{*}$ algebra over $\mathbb{C}, M$ is a closed, oriented Riemannian $G$-manifold and $E$ is a smooth $G$-bundle over $M$ whose fiber is a finitely generated projective Hilbert $G$ - $\mathcal{A}$-module $E_{0}$. Let $\mathcal{E}_{E}=L^{2}(E)$ be the completion of $C^{\infty}\left(M, \wedge_{\mathbb{C}} T^{*} M \otimes E\right)$ with respect to the scalar product

$$
\langle\xi, \eta\rangle=\int_{M}\langle\xi(x), \eta(x)\rangle .
$$

Here, we used a $G$-equivariant Riemannian structure on $M$ to get a Hilbert $G$ - $\mathcal{A}$ module structure on $\wedge_{\mathbb{C}} T_{x}^{*} M \otimes E_{x}, x \in M$.

Let $\xi, \eta \in \wedge_{\mathbb{C}} T_{x}^{*} M \otimes E_{x}$. Define $\xi^{*} \wedge \eta \in \wedge_{\mathbb{C}} T_{x}^{*} M \otimes \mathcal{A}$ by first letting

$$
\left(\alpha \otimes e_{1}\right)^{*} \wedge\left(\beta \otimes e_{2}\right)=\bar{\alpha} \wedge \beta \otimes\left\langle e_{1}, e_{2}\right\rangle, \quad \alpha, \beta \in \wedge_{\mathbb{C}} T_{x}^{*} M, e_{i} \in E_{x},
$$

$\left\langle e_{1}, e_{2}\right\rangle \in \mathcal{A}$, and then extending this to general elements $\xi$, $\eta$, where $\langle$.$\rangle is the scalar$ product on $E_{x}=E_{0}$.

We now define a signature element in $\mathcal{L}_{G, u}^{k}(\mathcal{A})$. Let $Q$ be the quadratic form on $\mathcal{E}_{E}$ given by

$$
Q(\xi, \eta)=i^{\partial \xi(n-\partial \xi)} \int_{M} \xi_{x}^{*} \wedge \eta_{x}
$$

for $\xi \in \mathcal{E}_{E}$ with homogeneous degree $\partial \xi$. Here $n$ is the dimension of $M . Q$ is equivariant since the metric and the scalar product $\langle$.$\rangle on E_{0}$ are equivariant. Let us check that $Q$ is regular, i.e., there exists an equivariant $\mathcal{A}$-bijection $T \in \mathcal{L}\left(\mathcal{E}_{E}\right)$ such that $\langle\xi, \eta\rangle=Q(\xi, T \eta)$ is a scalar product. Let

$$
(T \xi)_{x}=i^{-\partial \xi(n-\partial \xi)}\left(* \otimes I_{E}\right)\left(\xi_{x}\right), \quad \xi \in \mathcal{E}_{E} .
$$

Here $*$ is the Hodge operator, $*^{2} \alpha=(-1)^{\partial \alpha(n-\partial \alpha)} \alpha, \alpha \in \wedge_{\mathbb{C}} T^{*} M$. We have $T^{2} \xi=$ $i^{-2 \partial \xi(n-\partial \xi)}\left(*^{2} \otimes I_{E}\right) \xi=\xi$. Hence $T^{2}=I$. Clearly, $T$ is equivariant and $\mathcal{A}$-bijective. The quantity

$$
\langle\xi, \eta\rangle=Q(\xi, T \eta)=i^{\partial \xi(n-\partial \xi)} \int_{M} \xi_{x}^{*} \wedge(T \eta)_{x}=\int_{M} \xi_{x}^{*} \wedge\left(* \otimes I_{E}\right)\left(\eta_{x}\right)
$$

is evidently a scalar product. $Q$ also is a quadratic form: $Q(\xi, \eta)=Q(\eta, \xi)^{*}$ and $Q(\xi, \eta a)=Q(\xi, \eta) a, a \in \mathcal{A}$.

To use $\mathcal{E}_{E}$ and $Q$ to define an element in $\mathcal{L}_{G, u}^{k}(\mathcal{A})$, we need an operator $D$ satisfying the condition of Definition 2 . Let $\nabla$ be a metric-preserving antisymmetric (briefly, unitary) equivariant connection on $E$. Define an operator $D_{E}$ by

$$
D_{E}(\xi)=i^{\partial \xi} \nabla \xi, \quad \xi \in \mathcal{E} .
$$

In fact, let $\mathcal{H}_{\mathcal{A}}=L^{2}(G) \otimes_{\mathbb{C}} H_{\mathcal{A}}$ be the universal $G$ - $\mathcal{A}$-module [Bla]. By the equivariant stability theorem, $E_{0}$ can be embedded in $\mathcal{H}_{\mathcal{A}}$. Then $E$ can be constructed via a family of smooth equivariant projections $P=\left\{P_{x}\right\}_{x \in M}$ on $\mathcal{H}_{\mathcal{A}}$. Here each 
$P_{x}$ has a finitely generated projective range. In other words, $P: M \rightarrow \mathcal{L}\left(\mathcal{H}_{\mathcal{A}}\right)$ is smooth and the fiber $E_{x}=P_{x}\left(\mathcal{H}_{\mathcal{A}}\right)$. Then let

$$
(\nabla \xi)_{x}=\left(I_{\wedge_{\mathbb{C}} T_{x}^{*} M} \otimes P_{x}\right)\left(\left(d \otimes I_{\mathcal{H}_{\mathcal{A}}}\right) \xi\right)_{x}, \quad \xi \in C^{\infty}\left(M, \wedge_{\mathbb{C}} T^{*} M \otimes E\right) .
$$

The following lemma implies that $D_{E}$ satisfies the condition of Definition 2.

Lemma 8. With the above notation,

(1) $\forall \omega \in C^{\infty}\left(M, \wedge_{\mathbb{C}} T^{*} M\right)$ and $\xi \in \operatorname{dom}\left(D_{E}\right)$ we have $\omega \wedge \xi \in \operatorname{dom}\left(D_{E}\right)$ and $D_{E}(\omega \wedge \xi)=i^{\partial \xi+\partial \omega} d \omega \wedge \xi+i^{-\partial \omega} \omega \wedge D_{E} \xi$

(2) if $D_{E}^{\prime}$ is the conjugate of $D_{E}$ with respect to $Q$, then $D_{E}^{\prime}=-D_{E}$;

(3) $\mathrm{im}\left(D_{E}\right) \subset \operatorname{dom}\left(D_{E}\right)$ and $D_{E}^{2} \in \mathcal{L}_{G}\left(\mathcal{E}_{E}\right)$;

(4) there are equivariant $S_{1}$ and $S_{2}$ in $\mathcal{K}_{G}\left(\mathcal{E}_{E}\right)$ such that $\mathrm{im}\left(S_{2}\right) \subset \operatorname{dom}\left(D_{E}\right)$, $D_{E} S_{2}, S_{1} D_{E} \in \mathcal{L}_{G}\left(\mathcal{E}_{E}\right)$ and $S_{1} D_{E}+D_{E} S_{2}-I \in \mathcal{K}_{G}\left(\mathcal{E}_{E}\right)$.

Proof. (1) Let $\omega \in C^{\infty}\left(M, \wedge_{\mathbb{C}} T^{*} M\right), \xi \in \operatorname{dom}\left(D_{E}\right)$. Assume first that $\xi=\alpha \otimes a, \alpha \in$ $C^{\infty}\left(M, \wedge_{\mathbb{C}} T^{*} M\right)$ and $a \in E_{0}, P_{x} a=a$. Then

$$
\begin{aligned}
D_{E}(\omega \wedge \xi)_{x} & =i^{\partial \omega+\partial \alpha} \nabla(\omega \wedge \alpha \otimes a)_{x} \\
& =i^{\partial \omega+\partial \alpha}\left[\left(I \otimes P_{x}\right)\left(\left(d \omega \wedge \alpha+(-1)^{\partial \omega} \omega \wedge d \alpha\right) \otimes a\right)_{x}\right] \\
& =i^{\partial \omega+\partial \alpha}\left[\left(d \omega \wedge \alpha \otimes P_{x} a\right)+(-1)^{\partial \omega} \omega \wedge\left(I \otimes P_{x}\right)(d \alpha \otimes a)_{x}\right] \\
& =\left(i^{\partial \omega+\partial \alpha} d \omega \wedge \xi+i^{-\partial \omega} \omega \wedge D_{E} \xi\right)_{x} .
\end{aligned}
$$

Similarly, we can treat the general case.

(2) To show $D_{E}^{\prime}=-D_{E}$, it is enough to check that $Q\left(D_{E} \xi, \eta\right)=-Q\left(\xi, D_{E} \eta\right)$. Let $\xi=\alpha \otimes a, \eta=\beta \otimes b, \alpha, \beta \in C^{\infty}\left(M, \wedge_{\mathbb{C}} T^{*} M\right), a, b \in E_{0}, P_{x} a=a, P_{x} b=b, D_{E} \xi=$ $i^{\partial \alpha}(d \alpha \otimes P a), D_{E} \eta=i^{\partial \beta}(d \beta \otimes P b)$. Then

$$
\begin{aligned}
Q\left(D_{E} \xi, \eta\right) & =i^{3 \partial \xi+(\partial \alpha+1) \partial \beta} \int_{M}(\overline{d \alpha} \wedge \beta) \otimes\langle P a, b\rangle \\
& =-i^{(\partial \beta+1) \partial \alpha} i^{\partial \beta} \int_{M}(\bar{\alpha} \wedge d \beta) \otimes\langle a, P b\rangle \\
& =-Q\left(\xi, D_{E} \eta\right) .
\end{aligned}
$$

Similarly, one can check that $Q\left(D_{E} \xi, \eta\right)=-Q\left(\xi, D_{E} \eta\right)$ for the general case.

(3) Since the projection map $x \rightarrow P_{x}$ is smooth, $\operatorname{im}\left(D_{E}\right) \subset \operatorname{dom}\left(D_{E}\right)$. By the fact that the curvature $\nabla^{2}$ is an $\mathcal{L}_{G}\left(E_{0}\right)$-valued 2-form on $M, D_{E}^{2}$ is in $\mathcal{L}_{G}\left(\mathcal{E}_{E}\right)$.

(4) Let $E$ be constructed by a family of equivariant smooth $\mathcal{A}$-projections $P=$ $\left\{P_{x}\right\}_{x \in M}$ with finitely generated projective ranges: $E_{x}=P_{x} F_{0}$, where $F_{0}$ is a finitely generated $G$-A-projective Hilbert module. Then $D_{E}=P D_{F} P, D_{F}$ is the flat exterior differential on the trivial $G$ - $\mathcal{A}$-bundle $M \times F_{0}=F$. As in the ordinary case [Hil], $\left(I+D_{F}^{*} D_{F}+D_{F} D_{F}^{*}\right)^{-1} \in \mathcal{K}_{G}(\mathcal{F})$ and $D_{F}\left(I+D_{F}^{*} D_{F}+D_{F} D_{F}^{*}\right)^{-1} \in$ $\mathcal{K}_{G}(\mathcal{F})$ for $\mathcal{F}=L^{2}(F)$. Since the orthogonal complement $E^{\perp}$ of $E$ in $F$ is given by $(I-P) F$, and its connection $D_{E^{\perp}}$ is $(I-P) D_{F}(I-P)$, we have

$$
\begin{aligned}
D_{E} \oplus D_{E^{\perp}} & =P D_{F}-P D_{F}(I-P)+(I-P) D_{F}(I-P) \\
& =D_{F}-(I-P) D_{F}-(2 P-I) D_{F}(I-P)=D_{F}+A,
\end{aligned}
$$

where $A=(P-I) D_{F} P+(P) D_{F}(I-P)$. Let us first check that

$$
B_{1}=\left(D_{F}+A\right)\left(\lambda+\left(D_{F}+A\right)^{*}\left(D_{F}+A\right)+\left(D_{F}+A\right)\left(D_{F}+A\right)^{*}\right)^{-1} \in \mathcal{K}_{G}(\mathcal{F})
$$

and

$$
B_{2}=\left(\lambda+\left(D_{F}+A\right)^{*}\left(D_{F}+A\right)+\left(D_{F}+A\right)\left(D_{F}+A\right)^{*}\right)^{-1} \in \mathcal{K}_{G}(\mathcal{F}), \quad \lambda \rightarrow \infty .
$$


Now

$$
\begin{aligned}
(\lambda+ & \left.\left(D_{F}+A\right)^{*}\left(D_{F}+A\right)+\left(D_{F}+A\right)\left(D_{F}+A\right)^{*}\right)^{-1} \\
& =\left(I+\left(\lambda+D_{F}^{*} D_{F}+D_{F} D_{F}^{*}\right)^{-1}\right. \\
& \left.\times\left(D_{F}^{*} A+A D_{F}^{*}+A^{*} D_{F}+D_{F} A^{*}+A^{*} A+A A^{*}\right)\right)^{-1} \\
& \times\left(\lambda+D_{F}^{*} D_{F}+D_{F} D_{F}^{*}\right)^{-1},
\end{aligned}
$$

since the products of $A, A^{*}$ with $D_{F}$ and $D_{F}^{*}$ are bounded operators (by means of the argument of Sobolev spaces) and since the norm of the operator $B_{3}=$ $\left(\lambda+D_{F}^{*} D_{F}+D_{F} D_{F}^{*}\right)^{-1}\left(D_{F}^{*} A+A D_{F}^{*}+A^{*} D_{F}+D_{F} A^{*}+A^{*} A+A A^{*}\right)$ is less than 1 for sufficiently large $\lambda$. It follows that $B_{2} \in \mathcal{K}_{G}(\mathcal{F})$. Since $A$ is bounded, $A B_{2} \in \mathcal{K}_{G}(\mathcal{F})$ and $D_{F} B_{2}=D_{F}\left(I+B_{3}\right)^{-1}\left(\lambda+D_{F}^{*} D_{F}+D_{F} D_{F}^{*}\right)^{-1} \in \mathcal{K}_{G}(\mathcal{F})$.

Now let $S_{1}=\left(\lambda+D_{E}^{*} D_{E}+D_{E} D_{E}^{*}\right)^{-1} D_{E}^{*}$ and $S_{2}=D_{E}^{*}\left(\lambda+D_{E}^{*} D_{E}+D_{E} D_{E}^{*}\right)^{-1}$. Then $S_{1}$ extends to be in $\mathcal{K}_{G}\left(\mathcal{E}_{E}\right)$. Also $S_{2}$ is in $\mathcal{K}_{G}\left(\mathcal{E}_{E}\right)$. We have

$$
\begin{aligned}
S_{1} D_{E}+D_{E} S_{2}-I=\left(\lambda+D_{E}^{*} D_{E}+D_{E} D_{E}^{*}\right)^{-1} D_{E}^{*} D_{E} & +D_{E} D_{E}^{*}\left(\lambda+D_{E}^{*} D_{E}+D_{E} D_{E}^{*}\right)^{-1}-I \\
=- & \lambda\left(\lambda+D_{E}^{*} D_{E}+D_{E} D_{E}^{*}\right)^{-1}+\left[\left(\lambda+D_{E}^{*} D_{E}+D_{E} D_{E}^{*}\right)^{-1}, D_{E}^{*} D_{E}\right] \\
=- & \lambda\left(\lambda+D_{E}^{*} D_{E}+D_{E} D_{E}^{*}\right)^{-1}+\left(\lambda+D_{E}^{*} D_{E}+D_{E} D_{E}^{*}\right)^{-1} \\
& \times\left(D_{E}^{*} D_{E}^{2} D_{E}^{*}-D_{E}\left(D_{E}^{*}\right)^{2} D_{E}\right)\left(\lambda+D_{E}^{*} D_{E}+D_{E} D_{E}^{*}\right)^{-1} \in \mathcal{K}_{G}\left(\mathcal{E}_{E}\right) .
\end{aligned}
$$

Therefore, we have constructed an element $\left(\mathcal{E}_{E}, Q, D_{E}\right) \in \mathcal{L}_{G, u}^{0^{\prime}}(\mathcal{A})$ for $\operatorname{dim}(M)=$ $2 k$. If $\operatorname{dim}(M)=2 k+1$, we proceed as follows.

Let $\varepsilon(\xi)=(-1)^{\partial \xi} \xi$ for a homogeneous $\xi \in \mathcal{E}_{E}$. Then $\varepsilon$ extends to a grading on $\mathcal{E}_{E}$. Clearly, $\varepsilon T=-T \varepsilon$ (this is not true for $\operatorname{dim}(M)=2 k$ ), $\varepsilon D_{E}=-D_{E} \varepsilon$. We get $\left(\mathcal{E}_{E}, Q, D_{E}\right) \in \mathcal{L}_{G, u}^{1^{\prime}}(\mathcal{A})$. Hence, we can apply the maps $\psi_{i, u}$ in Definition 3 to the element $\left(\mathcal{E}_{E}, Q, D_{E}\right)$. Denote

$$
G-\operatorname{Sig}\left(D_{E}\right)=\psi_{i, u}\left(\left(\mathcal{E}_{E}, Q, D_{E}\right)\right) \in K_{i}^{G}(\mathcal{A}), \quad \operatorname{dim}(M)=2 k+i, i=0,1 .
$$

We call $G$-Sig $\left(D_{E}\right)$ the $G$-signature of $M$ with coefficients in $E$.

We now consider the central problem of this section. Let $E$ be a smooth $G$ - $\mathcal{A}$ bundle over $M$ whose fiber is a finitely generated projective Hilbert $G$ - $\mathcal{A}$-module $E_{0}$. As usual, $E$ defines an element $[E] \in K_{0}^{G}(\mathcal{A} \otimes C(M))$, where $C(M)$ is the space of all continuous functions on $M$. Let $\left[D_{M}\right]$ be the equivariant signature element in $K_{G}^{*}(C(M))$. Then

$$
\begin{aligned}
\psi(E, M) & \stackrel{\text { def }}{=}[E] \otimes_{C(M)}\left[D_{M}\right] \in K_{0}^{G}(\mathcal{A} \otimes C(M)) \otimes_{C(M)} K K_{*}^{G}(C(M), \mathbb{C}) \\
& \rightarrow K K_{*}^{G}(\mathbb{C}, \mathcal{A})=K_{*}^{G}(\mathcal{A}), *=0,1 .
\end{aligned}
$$

Here we need $\mathcal{A}$ to be $\sigma$-unital in order to use the Kasparov product.

Question. When is $\psi(E, M)$ a $G$-pseudo-equivalence invariant? Namely, let $h$ : $N \rightarrow M$ be an orientation-preserving $G$-pseudo-equivalence of closed, connected oriented Riemannian $G$-manifolds. When is $\psi\left(h^{*}(E), N\right)$ equal to $\psi(E, M)$ ?

Recall that $h$ is a $G$-pseudo-equivalence if $h$ is a homotopy equivalence and $G$ equivariant. The following observation is important: if $h$ is equivariantly homotopic to an orientation-preserving equivariant map $h_{1}: N \rightarrow M$, then $h^{*}(E)$ is isomorphic to $h_{1}^{*}(E)$. Hence $\left[h^{*}(E)\right]=\left[h_{1}^{*}(E)\right] \in K_{0}^{G}(\mathcal{A} \otimes C(N))$ (cf. [Hus], Thm. 4.7, p. 29). 
According to the $G$-smooth approximation theorem [Bre, Thm. 4.2, p. 317], $h$ is $G$-equivariantly homotopy equivalent to a smooth orientation-preserving $G$-pseudoequivalence $h_{1}: N \rightarrow M$. Therefore, it suffices to check $\psi\left(h_{1}^{*}(E), N\right)=\psi(E, M)$.

The following lemma then reduces the question for the even dimensional case to the $G$-pseudo-equivalence invariance of the $G$-signature $G$-Sig $\left(D_{E}\right)$ in $K_{0}^{G}(\mathcal{A})$.

Lemma 9. Let $\mathcal{A}$ be a unital $G-C^{*}$-algebra and $M$ be a closed oriented Riemannian $G$-manifold of even dimension. Suppose that $E$ is a smooth $G$-A-bundle over $M$ whose fiber is a finitely generated projective Hilbert $G$-A-module $E_{0} . \operatorname{Let}\left(\mathcal{E}_{E}, Q, D_{E}\right)$ be as in Lemma 8. Then

$$
G-\operatorname{Sig}\left(D_{E}\right)=[E] \otimes_{C(M)}\left[D_{M}\right] \in K_{0}^{G}(\mathcal{A}) .
$$

Proof. Recall that $[E] \in K K_{0}^{G}(\mathbb{C}, \mathcal{A} \otimes C(M))$ is defined by the Kasparov module $(\Gamma(E) \oplus 0, I, 0)$ and $\left[D_{M}\right] \in K K_{0}^{G}(C(M), \mathbb{C})$ is given by

$$
\left(L^{2}\left(M, T^{*} M\right), \varphi,\left(D_{M}+D_{M}^{*}\right)\left(I+\left(D_{M}+D_{M}^{*}\right)^{2}\right)^{-\frac{1}{2}}\right)
$$

where $\Gamma(E)$ is the space of continuous sections of $E$ and $\varphi$ is the multiplication by elements in $C(M)$. Assume first that $E$ is trivial, $E=M \times E_{0}$. Then $D_{E}=D_{M} \otimes I$ and $D_{E}+D_{E}^{*}=\left(D_{M}+D_{M}^{*}\right) \otimes I$. The Kasparov product of $[E]$ and $\left[D_{M}\right]$ is given by the following Kasparov bimodule:

$$
\begin{gathered}
\left(\left(\left(C(M) \otimes E_{0}\right) \oplus 0\right) \otimes_{C(M) \otimes \mathcal{A}}\left(L^{2}\left(M, T^{*} M\right) \otimes \mathcal{A}\right)\right. \\
\left.I,\left(D_{M}+D_{M}^{*}\right)\left(I+\left(D_{M}+D_{M}^{*}\right)^{2}\right)^{-\frac{1}{2}} \otimes I\right) .
\end{gathered}
$$

where $\left(\left(C(M) \otimes E_{0}\right) \oplus 0\right) \otimes_{C(M) \otimes \mathcal{A}}\left(L^{2}\left(M, T^{*} M\right) \otimes \mathcal{A}\right) \simeq L^{2}\left(M, T^{*} M \otimes E\right)$ with the inner product $\left\langle\left(x_{1}, x_{2}\right),\left(y_{1}, y_{2}\right)\right\rangle=\left\langle x_{2},\left\langle x_{1}, y_{1}\right\rangle_{1} y_{2}\right\rangle_{2}$ equivalent to the inner product on $L^{2}\left(M, T^{*} M \otimes E\right)$. This proves the assertion for the case of trivial bundles. In general, $E=P F$, where $F$ is a trivial smooth $G$ - $\mathcal{A}$-bundle over $M$ whose fiber is a finitely generated projective Hilbert $G$ - $\mathcal{A}$ module $F_{0}$ and $P: M \rightarrow \mathcal{L}\left(F_{0}\right)$ is a smooth family of projections. Then

$$
D_{E}=P D_{F} P, \quad D_{E}+D_{E}^{*}=P\left(D_{F}+D_{F}^{*}\right) P .
$$

Hence, $\left(D_{E}+D_{E}^{*}\right)\left(I+\left(D_{E}+D_{E}^{*}\right)^{2}\right)^{-\frac{1}{2}}=P\left(D_{F}+D_{F}^{*}\right)\left(I+\left(D_{F}+D_{F}^{*}\right)^{2}\right)^{-\frac{1}{2}} P$ is a $\left(D_{F}+D_{F}^{*}\right)\left(I+\left(D_{F}+D_{F}^{*}\right)^{2}\right)^{-\frac{1}{2}}$-connection of $\mathcal{E}_{E}$ (cf. [Bla], Prop. 18.3.3, p. 206). The result follows easily from definition of the Kasparov product.

Remark 4. (1) If $\mathcal{A}$ is not unital, then $K_{0}^{G}(\mathcal{A} \otimes C(M))=\operatorname{Ker}\left\{K_{0}^{G}\left(C(M) \otimes \mathcal{A}^{+}\right) \stackrel{i^{*}}{\rightarrow}\right.$ $\left.K_{0}^{G}(C(M) \otimes \mathbb{C})\right\}$ and $[E]=[((\Gamma(E) \oplus 0), I, 0)] \in \operatorname{Ker}\left(i^{*}\right)$, since $\Gamma(E) \otimes_{C(M) \otimes \mathcal{A}}$ $C(M) \otimes \mathbb{C}=0$, where $\mathcal{A}^{+}$is the $G$ - $C^{*}$-algebra obtained by adjoining an identity to $\mathcal{A}$. Note also that $\mathcal{E}_{E}$ can be considered as an $\mathcal{A}^{+}$-module and $\left[\left(\mathcal{E}_{E}, Q, D_{E}\right)\right]$ formed in the same manner as $G$-Sig $\left(D_{E}\right)$ is in $\operatorname{Ker}\left(K_{0}^{G}\left(\mathcal{A}^{+}\right) \rightarrow K_{0}^{G}(\mathbb{C})\right)$, i.e., this element is $G$-Sig $\left(D_{E}\right)$. With this in mind, we see that Lemma 9 holds for a $\sigma$-unital $\mathcal{A}$, namely,

$$
G-\operatorname{Sig}\left(D_{E}\right)=\left[\left(\mathcal{E}_{E}, Q, D_{E}\right)\right]=[E] \otimes_{C(M)}\left[D_{M}\right] .
$$

(2) Let $M$ be a closed oriented Riemannian $G$-manifold of odd dimension. As usual, the equivariant signature element $\left[D_{M}\right] \in K_{G}^{1}(C(M))$ can be defined as the restriction of $\left[D_{M \times S^{1}}\right]$ to the part $K_{G}^{1}(C(M))$ in the splitting of $K_{G}^{0}\left(C\left(M \times S^{1}\right)\right)$, where $G$ acts trivially on $S^{1}$. Thus the equivariant Novikov conjecture for the odd dimensional case is reduced to that for the even dimensional one. Hence, we will focus only on the even dimensional case. 
Clearly, $G$-Sig $\left(D_{E}\right)$ is independent of the connection on $E$, since any two connections on $E$ differ by a bounded operator in $\mathcal{L}_{G}\left(\mathcal{E}_{E}\right)$.

To consider the $G$-pseudo-equivalence invariance of $G$-Sig $\left(D_{E}\right)$, we need the following technical proposition.

Proposition 1. Let $\left(\mathcal{E}_{i}, Q_{i}, D_{i}\right) \in \mathcal{L}_{G, u}^{0}(\mathcal{A}), i=1,2$, and $R \in \mathcal{L}_{G}\left(\mathcal{E}_{1}, \mathcal{E}_{2}\right)$ satisfy

(a) $D_{i}^{\prime}=-D_{i}, D_{i}^{2}=0$;

(b) $R\left(\operatorname{dom}\left(D_{1}\right)\right) \subset \operatorname{dom}\left(D_{2}\right), R D_{1}=D_{2} R$;

(c) $R: \frac{\operatorname{Ker}\left(D_{1}\right)}{i m\left(D_{1}\right)} \rightarrow \frac{\operatorname{Ker}\left(D_{2}\right)}{i m\left(D_{2}\right)}$ is an isomorphism;

(d) there is an equivariant $S \in \mathcal{L}\left(\mathcal{E}_{1}\right)$ such that $S\left(\operatorname{dom}\left(D_{1}\right)\right) \subset \operatorname{dom}\left(D_{1}\right)$ and $I-R^{\prime} R=D_{1} S+S D_{1}$;

(e) there is an equivariant $\varepsilon \in \mathcal{L}\left(\mathcal{E}_{1}\right)$ such that $\varepsilon\left(\operatorname{dom}\left(D_{1}\right)\right) \subset \operatorname{dom}\left(D_{1}\right), \varepsilon D_{1}=$ $-D_{1} \varepsilon, \varepsilon^{2}=I, \varepsilon^{\prime}=\varepsilon$ and $\varepsilon\left(I-R^{\prime} R\right)=\left(I-R^{\prime} R\right) \varepsilon$.

Then $\psi_{0, u}\left(\left(\mathcal{E}_{1}, Q_{1}, D_{1}\right)\right)=\psi_{0, u}\left(\left(\mathcal{E}_{2}, Q_{2}, D_{2}\right)\right)$.

Proof. We can assume that $S^{\prime}=-S$. In fact, for $\xi, \eta \in \operatorname{dom}\left(D_{1}\right)$,

$$
Q_{1}\left(S^{\prime} \xi, D_{1} \eta\right)=Q_{1}\left(\xi, S D_{1} \eta\right)=Q_{1}\left(\xi,\left(I-R^{\prime} R\right) \eta\right)+Q_{1}\left(D_{1} \xi, S \eta\right) .
$$

It follows that $S^{\prime} \xi \in \operatorname{dom}\left(D_{1}\right), S^{\prime}\left(\operatorname{dom}\left(D_{1}\right)\right) \subset \operatorname{dom}\left(D_{1}\right)$ and

$$
I-R^{\prime} R=-\left(D_{1} S^{\prime}+S^{\prime} D_{1}\right) \text {. }
$$

Take $\tilde{S}=\frac{S-S^{\prime}}{2}$. We have $\tilde{S}\left(\operatorname{dom}\left(D_{1}\right)\right) \subset \operatorname{dom}\left(D_{1}\right)$ and $I-R^{\prime} R=D_{1} \tilde{S}+\tilde{S} D_{1} . \tilde{S}$ is obviously equivariant. Thus we can consider $\tilde{S}$ with $\tilde{S}^{\prime}=-\tilde{S}$.

Let $(\mathcal{E}, Q, D)=\left(\mathcal{E}_{1} \oplus \mathcal{E}_{2}, Q_{1} \oplus\left(-Q_{2}\right), D_{1} \oplus\left(-D_{2}\right)\right)$. By Lemma 6 ,

$$
\begin{aligned}
\psi_{0, u}((\mathcal{E}, Q, D)) & =\psi_{0, u}\left(\left(\mathcal{E}_{1}, Q_{1}, D_{1}\right)\right)+\psi_{0, u}\left(\left(\mathcal{E}_{2},-Q_{2},-D_{2}\right)\right) \\
& =\psi_{0, u}\left(\left(\mathcal{E}_{1}, Q_{1}, D_{1}\right)\right)-\psi_{0, u}\left(\left(\mathcal{E}_{2}, Q_{2}, D_{2}\right)\right) .
\end{aligned}
$$

Thus it suffices to prove $\psi_{0, u}((\mathcal{E}, Q, D))=0$.

For $t \in[0,1]$ we define $B_{t}(\xi, \eta)=Q\left(T_{t} \xi, T_{t} \eta\right), C_{t}(\xi, \eta)=Q\left(L_{t} \xi, \eta\right)$, where

$$
T_{t}=\left[\begin{array}{cc}
I & 0 \\
i t R \varepsilon & I
\end{array}\right], L_{t}=\left[\begin{array}{cc}
I-R^{\prime} R & (i \varepsilon+t S) R^{\prime} \\
R(i \varepsilon+t S) & I
\end{array}\right], \nabla_{t}=\left[\begin{array}{cc}
D_{1} & t R^{\prime} \\
0 & -D_{2}
\end{array}\right] \text {. }
$$

We have the following:

(i) $D T_{t}=T_{t} D$, since $\varepsilon D_{1}=-D_{1} \varepsilon$ and $R D_{1}=D_{2} R$.

(ii) $L_{t} \nabla_{t}=-\nabla_{t}^{\prime} L_{t}$. This follows from $I-R^{\prime} R=D_{1} S+S D_{1}, D_{1}^{2}=0$ and $D_{1} R^{\prime}=R^{\prime} D_{2}$. Note that by $D_{i}^{\prime}=-D_{i}$,

$$
\nabla_{t}^{\prime}=\left[\begin{array}{cc}
-D_{1} & 0 \\
-t R & D_{2}
\end{array}\right]
$$

(iii) $C_{t}\left(\nabla_{t} \xi, \eta\right)=Q\left(-\nabla_{t}^{\prime} L_{t} \xi, \eta\right)=-C_{t}\left(\xi, \nabla_{t} \eta\right)$. Hence $\nabla_{t}$ is antiselfadjoint with respect to $C_{t}(.,$.$) .$

(iv) Since $\varepsilon$ commutes with $R^{\prime} R, L_{0}=T_{1}^{\prime} T_{1}$ and

$$
T_{t}^{\prime}=\left[\begin{array}{cc}
I & i t \varepsilon R^{\prime} \\
0 & I
\end{array}\right]
$$

Let us check that $\left(\mathcal{E}, B_{t}, D\right) \in \mathcal{L}_{G, u}^{0}(\mathcal{A})$.

(a) $D$ is antisymmetric with respect to $B_{t}$. Indeed, by $(i), D T_{t}=T_{t} D$ and $D^{\prime}=-D$ we get $B_{t}(D \xi, \eta)=Q\left(D T_{t} \xi, T_{t} \eta\right)=Q\left(T_{t} \xi,-D T_{t} \eta\right)=B_{t}(\xi,-D \eta)$.

(b) Clearly, $D^{2} \in \mathcal{L}_{G}(\mathcal{E})$.

(c) Obviously, there are equivariant $R_{1}$ and $R_{2}$ in $\mathcal{L}_{G}(\mathcal{E})$ such that $R_{1} D+D R_{2}-$ $I \in \mathcal{K}_{G}(\mathcal{E})$, since $D_{i}$ has the corresponding property. 
(d) $B_{t}$ is associated with the invertible operator $T_{t} \in \mathcal{L}_{G}(\mathcal{E})$, and hence is a strongly nondegenerate quadratic form. In fact, the inverse of $T_{t}$ is

$$
T_{t}^{-1}=\left[\begin{array}{cc}
I & 0 \\
-i t R \varepsilon & I
\end{array}\right]
$$

$B_{t}$ is also regular with a compatible operator $T_{t}^{*} T T_{t}$, where $T$ is the operator compatible with $Q, T^{2}=I$. Indeed,

$$
\langle\xi, \eta\rangle_{B_{t}}=B_{t}\left(\xi, T_{t}^{*} T T_{t} \eta\right)=Q\left(T_{t} \xi, T_{t}\left(T_{t}^{*} T T_{t}\right) \eta\right)=\left\langle T_{t}^{*} T T_{t} \xi, T_{t}^{*} T T_{t} \eta\right\rangle .
$$

Therefore, $\left(\mathcal{E}, B_{t}, D\right) \in \mathcal{L}_{G, u}^{0}(\mathcal{A})$ for $0 \leq t \leq 1$. In other words, $\left(\mathcal{E}, B_{t}, D\right) \in$ $\mathcal{L}_{G, u}^{0}(C([0,1], \mathcal{A}))$.

Let us now check that $\left(\mathcal{E}, C_{t}, \nabla_{t}\right) \in \mathcal{L}_{G, u}^{0}(\mathcal{A})$ for $t$ near 0 . Since $T_{t}$ is invertible and $L_{0}=T_{1}^{\prime} T_{1}, L_{0}$ is also invertible. It follows that $L_{t}$ is invertible for $t$ near 0 , say $0 \leq t \leq t_{0}$. By $($ iii $), \nabla_{t}$ is antisymmetric with respect to $C_{t}$ and $\nabla_{t}^{2}=0$, since $D_{1} R^{\prime}=R^{\prime} D_{2}$ and $D_{i}^{2}=0$. Let $S_{i}, \tilde{S}_{i} \in \mathcal{K}_{G}\left(\mathcal{E}_{i}\right)$ be such that $S_{i} D_{i}+D_{i} \tilde{S}_{i}-I \in$ $\mathcal{K}_{G}\left(\mathcal{E}_{i}\right), S_{i} D_{i} \in \mathcal{L}_{G}\left(\mathcal{E}_{i}\right), i m\left(\tilde{S}_{i}\right) \subset \operatorname{dom}\left(D_{i}\right), D_{i} \tilde{S}_{i} \in \mathcal{L}_{G}\left(\mathcal{E}_{i}\right), i=0,1$. Then

$$
\begin{gathered}
{\left[\begin{array}{cc}
S_{1} & 0 \\
0 & -S_{2}
\end{array}\right]\left[\begin{array}{cc}
D_{1} & t R^{\prime} \\
0 & -D_{2}
\end{array}\right]+\left[\begin{array}{cc}
D_{1} & t R^{\prime} \\
0 & -D_{2}
\end{array}\right]\left[\begin{array}{cc}
\tilde{S}_{1} & 0 \\
0 & -\tilde{S}_{2}
\end{array}\right]-\left[\begin{array}{ll}
I & 0 \\
0 & I
\end{array}\right]} \\
=\left[\begin{array}{cc}
S_{1} D_{1}+D_{1} \tilde{S}_{1}-I & t S_{1} R^{\prime}-t R^{\prime} \tilde{S}_{2} \\
0 & S_{2} D_{2}+D_{2} \tilde{S}_{2}-I
\end{array}\right] \in \mathcal{K}_{G}(\mathcal{E}),
\end{gathered}
$$

since $S_{1} \in \mathcal{K}_{G}\left(\mathcal{E}_{1}\right), \tilde{S}_{2} \in \mathcal{K}_{G}\left(\mathcal{E}_{2}\right)$ and $R^{\prime} \in \mathcal{L}_{G}\left(\mathcal{E}_{1}, \mathcal{E}_{2}\right)$. Note that $\langle\xi, \eta\rangle_{C_{t}} \stackrel{\text { def }}{=}$ $C_{t}\left(\xi, T L_{t} \eta\right)=Q\left(L_{t} \xi, T L_{t} \eta\right)=\left\langle L_{t} \xi, L_{t} \eta\right\rangle$. We have that $C_{t}$ is a strongly nondegenerate and regular quadratic form. Thus we conclude that $\left(\mathcal{E}_{t}, C_{t}, \nabla_{t}\right)$ is in $\mathcal{L}_{G, u}^{0}(\mathcal{A})$ for $0 \leq t \leq t_{0}$.

Now $C_{0}(\xi, \eta)=Q\left(L_{0} \xi, \eta\right)=Q\left(T_{1}^{\prime} T_{1} \xi, \eta\right)=B_{1}(\xi, \eta)$ and $\nabla_{0}=D$. We have $\left(\mathcal{E}, B_{1}, D\right)=\left(\mathcal{E}, C_{0}, \nabla_{0}\right)$. Hence,

$$
\psi_{0, u}((\mathcal{E}, Q, D))=\psi_{0, u}\left(\left(\mathcal{E}, B_{0}, D\right)\right)=\psi_{0, u}\left(\left(\mathcal{E}, B_{1}, D\right)\right)=\psi_{0, u}\left(\left(\mathcal{E}, C_{t_{0}}, \nabla_{t_{0}}\right)\right) .
$$

Hence it suffices to show that $\psi_{0, u}\left(\left(\mathcal{E}, C_{t_{0}}, \nabla_{t_{0}}\right)\right)=0$. By Lemma 6 , we need only to check that $\operatorname{im}\left(\nabla_{t_{0}}\right)=\operatorname{ker}\left(\nabla_{t_{0}}\right)$.

Obviously, $\nabla_{t_{0}}^{2}=0, i m\left(\nabla_{t_{0}}\right) \subset \operatorname{ker}\left(\nabla_{t_{0}}\right)$. To show that $\operatorname{Ker}\left(\nabla_{t_{0}}\right) \subset i m\left(\nabla_{t_{0}}\right)$, let $\left(\xi_{1}, \xi_{2}\right) \in \mathcal{E}$ is such that $\nabla_{t_{0}}\left(\xi_{1}, \xi_{2}\right)=0$. By condition $(c), R^{\prime}: \frac{\operatorname{Ker}\left(D_{2}\right)}{i m\left(D_{2}\right)} \rightarrow \frac{\operatorname{Ker}\left(D_{1}\right)}{i m\left(D_{1}\right)}$ is bijective. Then there exists $\xi_{2}^{\prime} \in \mathcal{E}_{2}$ such that $\xi_{2}=-D_{2} \xi_{2}^{\prime}$, since $-t_{0} R^{\prime} \xi_{2}=$ $D_{1} \xi_{1}=0$ in $\frac{\operatorname{Ker}\left(D_{1}\right)}{\operatorname{im}\left(D_{1}\right)}$. This implies that $D_{1} \xi_{1}=-t_{0} R^{\prime} \xi_{2}=t_{0} R^{\prime} D_{2} \xi_{2}^{\prime}=t_{0} D_{1} R^{\prime} \xi_{2}^{\prime}$. Hence $\xi_{1}-t_{0} R^{\prime} \xi_{2}^{\prime} \in \operatorname{Ker} D_{1}$. The bijectivity of $R^{\prime}$ implies that there exists $\left[\xi_{2}^{\prime \prime}\right] \in$ $\frac{\operatorname{Ker}\left(D_{2}\right)}{\operatorname{im}\left(D_{2}\right)}$ such that $t_{0} R^{\prime}\left(\xi_{2}^{\prime \prime}\right)=\xi_{1}-t_{0} R^{\prime} \xi_{2}^{\prime}$. Thus there exists $\xi_{1}^{\prime} \in \mathcal{E}_{1}$ such that $\xi_{1}-t_{0} R^{\prime}\left(\xi_{2}^{\prime}+\xi_{2}^{\prime \prime}\right)=D_{1} \xi_{1}^{\prime}$. Note that $D_{1} \xi_{2}^{\prime \prime}=0$. We get $\xi_{1}=t_{0} R^{\prime}\left(\xi_{2}^{\prime}+\xi_{2}^{\prime \prime}\right)+D_{1} \xi_{1}^{\prime}$ and $\xi_{2}=-D_{2}\left(\xi_{2}^{\prime}+\xi_{2}^{\prime \prime}\right)$. We have thus found $\left(\xi_{1}^{\prime}, \xi_{2}^{\prime \prime \prime}\right) \in \mathcal{E}$ such that $\nabla_{t_{0}}\left(\xi_{1}^{\prime}, \xi_{2}^{\prime \prime \prime}\right)=$ $\left(\xi_{1}, \xi_{2}\right)$. Therefore, $i m\left(\nabla_{t_{0}}\right)=\operatorname{ker}\left(\nabla_{t_{0}}\right)$.

The idea of the above proof is first to transform the equality of two elements $\psi_{0, u}\left(\mathcal{E}_{i}, Q_{i}, D_{i}\right)$ to the vanishing of $\psi_{0, u}(\mathcal{E}, Q, D)$, where $(\mathcal{E}, Q, D)$ is the direct sum formed in the proof. Then we construct a path of $\left(\mathcal{E}, C_{t}, \nabla_{t}\right)$ in $\mathcal{L}_{G, u}^{0}(\mathcal{A}), 0 \leq$ $t \leq t_{0}$, such that $\left(\mathcal{E}, C_{0}, \nabla_{0}\right)=(\mathcal{E}, Q, D)$ and $\left(\mathcal{E}, C_{t_{0}}, \nabla_{t_{0}}\right)$ satisfies $i m\left(\nabla_{t_{0}}\right)=$ $\operatorname{Ker}\left(\nabla_{t_{0}}\right)$. By homotopy invariance of $\psi_{0, u}(\mathcal{E}, Q, D)$ and Lemma 6 , we prove that $\psi_{0, u}(\mathcal{E}, Q, D)=\psi_{0, u}\left(\mathcal{E}, C_{t_{0}}, \nabla_{t_{0}}\right)=0$. 
We now use Proposition 1 to prove the $G$-pseudo-equivalence invariance of $G$ $\operatorname{Sig}\left(D_{E}\right)$ for a flat $G$-A-bundle $E$. Our goal in the rest of this section is to check the condition of Proposition 1.

Let $\mathcal{A}$ be a $G-C^{*}$-algebra, $N$ and $M$ two closed, oriented Riemannian $G$-manifolds and $E$ a $G$-A-bundle over $M$ whose fiber is a finitely generated projective Hilbert $G$-A-module $E_{0} . E$ is said to be $G$-unitary flat if $E$ is furnished with an equivariant scalar product and an equivariant unitary flat connection. Let $f: N \rightarrow M$ be an equivariant smooth map. It is clear that if $E$ is a $G$-unitary flat bundle, then so is $f^{*}(E)$. If $f$ and $f_{1}$ are two equivariant smooth maps from $N$ into $M$ which are $G$-equivariantly homotopic via a $G$-homotopy $H$, then $H^{*}: f^{*}(E) \rightarrow f_{1}^{*}(E)$ induces an isomorphism. Suppose that $\nabla$ is an equivariant unitary flat connection on $E$ and $D_{E} \xi=i^{\partial \xi} \nabla \xi$; then $D_{f^{*}(E)}=D_{f_{1}^{*}(E)}$. Observe that the map $f^{*}$ : $C^{\infty}\left(M, \wedge_{\mathbb{C}} T^{*} M \otimes E\right) \rightarrow C^{\infty}\left(N, \wedge_{\mathbb{C}} T^{*} N \otimes f^{*}(E)\right)$ need not be extendable to a bounded operator from $\mathcal{E}_{E}=L^{2}(E)$ to $\mathcal{E}_{f^{*}(E)}=L^{2}\left(f^{*}(E)\right)$, since $f^{*}$ is not closed in general. To get around this problem, we follow $[\mathrm{HiS}]$ and use embeddings.

Let $\omega \in \wedge_{\mathbb{C}} T^{*} N$. Define $e_{\omega}: \wedge_{\mathbb{C}} T^{*} N \rightarrow \wedge_{\mathbb{C}} T^{*} N$ by $e_{\omega}(\alpha)=\alpha \wedge \omega$.

Lemma 10 ([HiS]). Let $M$ and $N$ be closed, oriented Riemannian G-manifolds.

(a) Suppose $h: N \rightarrow M$ is a smooth equivariant map and $\omega \in \wedge_{\mathbb{C}} T^{*} N$ is equivariant. Let $N_{0}$ be the support of $\omega$ in $N$ and $h$ be a G-submersion from a neighborhood of $N_{0}$ into $M$. Then $e_{\omega} h^{*}: \mathcal{E}_{E} \rightarrow \mathcal{E}_{h^{*}(E)}$ is an equivariant operator in $\mathcal{L}\left(\mathcal{E}_{E}, \mathcal{E}_{h^{*}(E)}\right)$, where $E$ is a $G$-A-unitary flat bundle over $M$, and the norm of $e_{\omega} h^{*}$ is independent of $E$.

(b) Assume $h: N \times[0,1] \rightarrow M$ is a smooth equivariant map and $\omega \in \wedge_{\mathbb{C}} T^{*} N$ is a closed equivariant form on $N$, where $G$ acts trivially on $[0,1]$. Suppose $h$ (resp., $\left.h_{0}, h_{1}\right)$ is a $G$-submersion from a neighborhood of $N_{0} \times[0,1]$ (resp., $N_{0}$ ) into $M$. If $\nabla$ is an equivariant unitary flat connection on the $G$-A-unitary flat bundle $E$ over $M$, then there exists an equivariant operator $S \in \mathcal{L}_{G}\left(\mathcal{E}_{E}, \mathcal{E}_{h^{*}(E)}\right)$ such that

$$
e_{\omega} h_{1}^{*}-e_{\omega} h_{0}^{*}=h_{0}^{*}(\nabla) S+S \nabla .
$$

Proof. (a) Since $e_{\omega} h^{*}$ is equivariant, it suffices to show the boundedness of $e_{\omega} h^{*}$. The proof is the same as that of Lemma 3.2 in [HiS]. We provide more details below.

Using a partition of unity, we see that the assertion is a local statement. Thus let $N=O \times M$ and $E$ be trivial. $h: N \rightarrow M$ is then a projection. Let $\alpha d y_{I} \otimes \xi \in$ $\wedge_{\mathbb{C}} T^{*} M \otimes E_{0}$. We have

$$
\begin{gathered}
h^{*}\left(\alpha d y_{I} \otimes \xi\right)_{(x, y)}=\alpha(h(x, y)) d h_{I} \otimes h^{*}(\xi)_{(x, y)}=\alpha(y) d y_{I} \otimes \xi, \\
e_{\omega} h^{*}\left(\alpha d y_{I} \xi\right)_{(x, y)}=\alpha(y) d y_{I} \wedge \omega(x, y) \otimes \xi .
\end{gathered}
$$

Moreover,

$$
\begin{aligned}
\left\|e_{\omega} h^{*}\left(\alpha d y_{I} \otimes \xi\right)\right\|^{2} & =\left\|\int_{N} \overline{\left(\alpha(y) d y_{I} \wedge \omega(x, y)\right)} \wedge *\left(\alpha(y) d y_{I} \wedge \omega(x, y)\right) \otimes\langle\xi, \xi\rangle\right\|^{2} \\
& =\left\|\alpha d y_{I} \wedge \omega\right\|^{2}\|\langle\xi, \xi\rangle\|^{2} \leq\|\omega\|^{2}\left\|\alpha d y_{I} \otimes \xi\right\|^{2}
\end{aligned}
$$

Hence, $\left\|e_{\omega} h^{*}\right\| \leq\|\omega\|$.

(b) Recall that the contraction $i_{\frac{\partial}{\partial t}}$ is defined by

$$
i_{\frac{\partial}{\partial t}}\left(\alpha d x_{I} \wedge d t\right)=(-1)^{|I|} \alpha d x_{I} .
$$


$i_{\frac{\partial}{\partial t}}$ is equivariant since $G$ acts trivially on $[0,1]$. Define, for $\omega_{1} \in \mathcal{E}_{E}$,

$$
S\left(\omega_{1}\right)=\int_{0}^{1} i_{\frac{\partial}{\partial t}}\left(e_{\omega} h^{*}\left(\omega_{1}\right)\right) d t \in L^{2}\left(N, \wedge_{\mathbb{C}} T^{*} N \otimes h_{0}^{*}(E)\right) .
$$

Here $\omega$ is considered as a smooth form on $N \times[0,1]$. By part $(a), e_{\omega} h^{*}$ is bounded, and $i_{\frac{\partial}{\partial t}}$ is also bounded. $S$ is thus bounded. Clearly, $S$ is equivariant.

Since $e_{\omega} h_{1}^{*}-e_{\omega} h_{0}^{*}=\int_{0}^{1} \frac{\partial}{\partial t}\left(e_{\omega} h^{*}\right) d t$, it is enough to show locally that

$$
h_{0}^{*}(\nabla) S+S \nabla=\int_{0}^{1} \frac{\partial}{\partial t}\left(e_{\omega} h^{*}\right) d t .
$$

Let $\omega_{1}=\sum_{I} \alpha_{I} d y_{I} \otimes \xi, d y_{I}=d y_{i_{1}} \wedge \ldots \wedge d y_{i_{I}}$. We have

$$
\begin{aligned}
e_{\omega} h^{*} & \left(\omega_{1}\right)=h^{*}\left(\omega_{1}\right) \wedge \omega=h^{*}\left(\omega_{1}\right) \wedge \omega(z, y, t) \\
= & \sum_{I} \alpha_{I}(h(z, y, t)) \wedge_{i \in I}\left(\sum_{k} h_{i, z_{k}} d z_{k}+\sum_{j} h_{i, y_{j}} d y_{j}+h_{i, t} d t\right) \wedge \omega(z, y, t) \otimes \xi,
\end{aligned}
$$

where $h: O \times M \times[0,1] \rightarrow M, z$ and $y$ are the variables of $O$ and $M$, resp., and $h_{i, z_{k}}, h_{i, y_{j}}$ and $h_{i, t}$ are the derivatives of $h_{i}$ with respect to $z_{k}, y_{j}$ and $t$, respectively. Then

$$
\frac{\partial}{\partial t}\left(e_{\omega} h^{*}\left(\omega_{1}\right)\right) d t=\frac{\partial}{\partial t}\left[\sum_{I} \alpha_{I}(h) \wedge_{i \in I}\left(\sum_{k} h_{i, z_{k}} d z_{k}+\sum_{j} h_{i, y_{j}} d y_{j}\right) \wedge \omega\right] d t \otimes \xi
$$

$$
\begin{gathered}
=\sum_{I}\left\{\sum_{s} \alpha_{I, \alpha}(h) h_{s, t} \wedge_{i \in I}\left(\sum_{k} h_{i, z_{k}} d z_{k}+\sum_{j} h_{i, y_{j}} d y_{j}\right) \wedge \omega \wedge d t \otimes \xi\right. \\
+\alpha_{I}(h) \wedge_{l=1}^{I}(-1)^{l-1}\left(\sum_{k} h_{i_{l}, z_{k}, t} d z_{k}+\sum_{j} h_{i_{l}, y_{j}, t} d y_{j}\right) \\
\left.\times \wedge_{i \in I, i \neq i_{l}}\left(\sum_{k} h_{i, z_{k}} d z_{k}+\sum_{j} h_{i, y_{j}} d y_{j}\right) \wedge \omega d t \otimes \xi\right\} .
\end{gathered}
$$

Since $\nabla$ is flat, we can assume locally that $\nabla=d \otimes p$, where $p$ is locally a constant projection. We have

$$
\begin{gathered}
\nabla\left(\omega_{1}\right)=d\left(\sum_{I} \alpha_{I} d y_{I}\right) \otimes \xi=\sum_{I, s} \alpha_{I, s} d y_{s} \wedge d y_{I} \otimes \xi \\
h^{*} \nabla\left(\omega_{1}\right)=\sum_{I, s} \alpha_{I, s}(h(z, y, t)) d h_{s} \wedge_{i \in I} d h_{i} \otimes \xi \\
e_{\omega} h^{*} \nabla\left(\omega_{1}\right)=\sum_{I}\left[\sum_{s} \alpha_{I, s}(h(z, y, t))\left(\sum_{k} h_{s, z_{k}} d z_{k}+\sum_{j} h_{s, y_{j}} d y_{j}+h_{s, t} d t\right)\right. \\
\left.\times \wedge_{i \in I}\left(\sum_{k} h_{i, z_{k}} d z_{k}+\sum_{j} h_{i, y_{j}} d y_{j}+h_{i, t} d t\right) \wedge \omega(z, y, t) \otimes \xi\right],
\end{gathered}
$$




$$
\begin{aligned}
\int_{0}^{1}\left[i_{\frac{\partial}{\partial t}}\right. & \left.\left(e_{\omega} h^{*} \nabla\left(\omega_{1}\right)\right)\right] d t \\
= & \int_{0}^{1} \sum_{I}\left\{\sum _ { s } \alpha _ { I , s } ( h ) \left[-\left(\sum_{k} h_{s, z_{k}} d z_{k}+\sum_{j} h_{s, y_{j}} d y_{j}\right)\right.\right. \\
& \wedge_{l=1}^{I}(-1)^{l-1} \wedge_{i \in I, i \neq i_{l}}\left(\sum_{k} h_{i, z_{k}} h_{i_{l}, t} d z_{k}+\sum_{j} h_{i, y_{j}} h_{i_{l}, t} d y_{j}\right) \\
& \left.\left.+\wedge_{i \in I}\left(\sum_{k} h_{i, z_{k}} h_{s, t} d z_{k}+\sum_{j} h_{i, y_{j}} h_{s, t} d y_{j}\right)\right]\right\} \wedge \omega \wedge d t \otimes \xi .
\end{aligned}
$$

Now since $d \omega=0$,

$$
\begin{aligned}
h_{0}^{*}(\nabla) & \left(\int_{0}^{1}\left(i_{\frac{\partial}{\partial t}}\left(e_{\omega} h^{*}\left(\omega_{1}\right)\right)\right) d t\right) \\
= & \int_{0}^{1} \nabla\left(\sum _ { I } \alpha _ { I } ( h ) \wedge _ { l = 1 } ^ { I } ( - 1 ) ^ { l - 1 } \wedge _ { i \in I , i \neq i _ { l } } \left(\sum_{k} h_{i, z_{k}} h_{i_{l}, t} d z_{k}\right.\right. \\
& \left.\left.+\sum_{j} h_{i, y_{j}} h_{i_{l}, t} d y_{j}\right) \wedge \omega \otimes \xi\right) d t \\
= & \int_{0}^{1}\left\{\sum _ { I } \left(\sum_{s} \alpha_{I, s}(h) d h_{s} \wedge_{l=1}^{I}(-1)^{l-1}\right.\right. \\
& \times \wedge_{i \in I, i \neq i_{l}}\left(\sum_{k} h_{i, z_{k}} h_{i_{l}, t} d z_{k}+\sum_{j} h_{i, y_{j}} h_{i_{l}, t} d y_{j}\right) \wedge \omega \\
+ & \alpha_{I}(h) \wedge_{l=1}^{I}(-1)^{l-1}\left(\sum_{k} h_{i_{l}, t, z_{k}} d z_{k}+\sum_{j} h_{i_{l}, t, y_{j}} d y_{j}\right) \\
\times & \left.\left.\wedge_{i \in I, i \neq i_{l}}\left(\sum_{k} h_{i, z_{k}} d z_{k}+\sum_{j} h_{i, y_{j}} d y_{j}\right) \wedge \omega\right)\right\} d t \otimes \xi .
\end{aligned}
$$

Adding (3) and (4) together and using (2), we get

$$
\left(h_{0}^{*}(\nabla) S+S \nabla\right)\left(\omega_{1}\right)=\int_{0}^{1} \frac{\partial}{\partial t}\left(e_{\omega} h^{*}\left(\omega_{1}\right)\right) d t .
$$

Let $h_{!, \omega}$ be the conjugate of $e_{\omega} h^{*}$ with respect to the quadratic form $Q$, where $h: N \rightarrow M$ is a submersion in a neighborhood of the support of $\omega$. Locally, we can find a formula for $h_{!, \omega}$. Let $h: \mathbb{R}^{m} \times \mathbb{R}^{k} \rightarrow \mathbb{R}^{m}$ be the projection. If $\omega$ is a continuous function with compact support in $\mathbb{R}^{m} \times \mathbb{R}^{k}$, then for $\alpha d x_{I} \otimes \xi \in \wedge_{\mathbb{C}} T^{*} \mathbb{R}^{m}$ we have $e_{\omega} h^{*}\left(\alpha d x_{I} \otimes \xi\right)=\alpha(x) d x_{I} \wedge \omega \otimes \xi$. For $\left|J_{1}\right|=k$ we can calculate

$$
\begin{aligned}
& Q\left(\beta d x_{I_{1}} \wedge d y_{J_{1}} \otimes \eta, e_{\omega} h^{*}\left(\alpha d x_{I} \otimes \xi\right)\right) \\
& \quad=i^{\left(m-\left|I_{1}\right|\right) k} Q\left(h_{!, \omega}\left(\beta d x_{I_{1}} \wedge d y_{J_{1}} \otimes \eta\right), \alpha d x_{I} \otimes \xi\right),
\end{aligned}
$$

with

$$
h_{!, \omega}\left(\beta d x_{I_{1}} \wedge d y_{J_{1}} \otimes \eta\right)=i^{\left(m-\left|I_{1}\right|\right)\left|J_{1}\right|} d x_{I_{1}} \int \bar{\omega}(x, y) \beta(x, y) d y_{J_{1}} \otimes \eta,
$$

and $h_{!, \omega}=0$ for $\left|J_{1}\right| \neq k$. 
Let $G$ act on $\mathbb{R}^{k}$ by isometries, and let $B^{k}$ be the equivariant unit open ball in $\mathbb{R}^{k}$. Suppose that $P: N \times B^{k} \rightarrow M$ is an equivariant submersion and $\nu_{k}$ is an equivariant volume form of mass 1 on $B^{k}$. Let $q: N \times B^{k} \rightarrow N$ and $\pi_{k}: N \times B^{k} \rightarrow B^{k}$ be the projections. $\omega=\pi_{k}^{*}\left(\nu_{k}\right)$ and $P_{0}=\left.P\right|_{N \times\{0\}}$. Define

$$
R_{p, \nu_{k}}=q ! e_{\omega} P^{*} \in \mathcal{L}_{G}\left(\mathcal{E}_{E}, \mathcal{E}_{P_{0}^{*}(E)}\right),
$$

where $q_{!}: \mathcal{E}_{P^{*}(E)} \rightarrow \mathcal{E}_{P_{0}^{*}(E)}$ is the pushforward. The following lemma is crucial in verifying the condition of Proposition 1.

Lemma 11. (a) Let $P^{\prime}: N \times B^{l} \rightarrow M$ be another equivariant submersion and $\nu_{l}$ be an equivariant volume form of mass 1 on $B^{l}$. If the maps $P(x, o)$ and $P^{\prime}(x, o)$ are equivariantly homotopic, then there exists an $S \in \mathcal{L}_{G}\left(\mathcal{E}_{E}, \mathcal{E}_{P_{0}^{*}(E)}\right)$ such that

$$
D_{E} S+S D_{E}=R_{p, \nu_{k}}-R_{p^{\prime} \nu_{l}} .
$$

(b) Let $P^{\prime}: O \times B^{l} \rightarrow N$ be an equivariant submersion and $\nu_{l}$ an equivariant volume form of mass 1 on $B^{l}$, where $O$ is a compact oriented Riemannian $G$ manifold. If $P^{\prime \prime}: O \times B^{l} \times B^{k} \rightarrow M$ is given by $P^{\prime \prime}(z, s, t)=P\left(P^{\prime}(z, s), t\right)$ and $\nu=\nu_{l} \times \nu_{k}$, then $R_{p^{\prime}, \nu_{l}} R_{p, \nu_{k}}=R_{p^{\prime \prime}, \nu}$.

Proof. Since the volume forms $\nu_{k}, \nu_{l}$ and $\nu$ have compact supports, there exists an equivariant $\varphi \in C_{c}^{\infty}\left(B^{k}\right)$ such that $\varphi \nu_{k}=\nu_{k}$. As a consequence, $R_{p, \nu_{k}}=q_{!, \varphi} e_{\omega} P^{*}$.

(a) Let $\tilde{P}: N \times B^{k} \times B^{l} \times B^{r} \times[0,1] \rightarrow M$ be an equivariant submersion such that $\tilde{P}(x, s, t, u, 0)=P(x, s)$ and $\tilde{P}(x, s, t, u, 1)=P^{\prime}(x, t)$. This $\tilde{P}$ exists since $P$ and $P^{\prime}$ are submersions and homotopic. Let $\nu_{r}$ be a volume form of mass 1 on $B^{r}$ with compact support. Define $\tilde{P}_{i}: N \times B^{k} \times B^{l} \times B^{r} \rightarrow M$ by $\tilde{P}_{i}(z)=$ $\tilde{P}(z, i), z \in N \times B^{k} \times B^{l} \times B^{r}, i=0,1$. Then $R_{p, \nu_{k}}=R_{\tilde{p}_{0}, \nu_{k} \times \nu_{l} \times \nu_{r}}=q_{!}^{k, l, r} e_{\tilde{\omega}} \tilde{P}_{0}^{*}$ and $R_{p^{\prime}, \nu_{l}}=R_{\tilde{p}_{1}, \nu_{k} \times \nu_{l} \times \nu_{r}}=q_{!}^{k, l, r} e_{\tilde{\omega}} \tilde{P}_{1}^{*}$, where $q^{k, l, r}: N \times B^{k} \times B^{l} \times B^{r} \rightarrow N, \pi_{k, l, r}:$ $N \times B^{k} \times B^{l} \times B^{r} \rightarrow B^{k} \times B^{l} \times B^{r}$ are projections and $\tilde{\omega}=\left(\pi_{k, l, r}\right)^{*}\left(\nu_{k} \times \nu_{l} \times \nu_{r}\right)$. By Lemma 10 , there is an equivariant $S_{1} \in \mathcal{L}_{G}\left(\mathcal{E}_{E}, \mathcal{E}_{\tilde{P}_{0}^{*}(E)}\right)$ such that

$$
q_{!}^{k, l, r}\left(e_{\tilde{\omega}} \tilde{P}_{1}^{*}-e_{\tilde{\omega}} \tilde{P}_{0}^{*}\right)=q_{!}^{k, l, r}\left(\tilde{P}_{0}^{*}(D) S_{1}+S_{1} D\right)=D\left(q_{!}^{k, l, r} S_{1}\right)+\left(q_{!}^{k, l, r} S_{1}\right) D .
$$

Hence, $R_{p^{\prime}, \nu_{l}}-R_{p, \nu_{k}}=D S+S D, S=q_{!}^{k, l, r} S_{1}$.

(b) Let $\bar{P}: O \times B^{k} \times B^{l} \rightarrow N \times B^{k}, \bar{q}: O \times B^{k} \times B^{l} \rightarrow O \times B^{l}$ and $q^{\prime}: O \times B^{l} \rightarrow O$ be defined by

$$
\bar{P}(z, s, t)=\left(P^{\prime}(z, t), s\right), \quad \bar{q}(z, s, t)=(z, t), \quad q^{\prime}(z, t)=z .
$$

Let $\pi_{k, l}: O \times B^{k} \times B^{l} \rightarrow B^{k} \times B^{l}$ and $\pi_{l}: O \times B^{k} \times B^{l} \rightarrow B^{l}$ be the projections and $\tilde{\omega}_{1}=\left(\pi_{k, l}\right)^{*}\left(\nu_{k} \times \nu_{l}\right), \omega_{1}=\left(\pi_{l}\right)^{*}\left(\nu_{l}\right)$. Then for the projection $q^{0}: O \times B^{k} \times B^{l} \rightarrow O$,

$$
R_{p^{\prime \prime}, \nu}=q_{!}^{0} e_{\tilde{\omega}_{1}}\left(P^{\prime \prime}\right)^{*}=q_{!}^{\prime} \bar{q}_{!} e_{\omega_{1}} \bar{P}^{*} e_{\omega} P^{*} .
$$

This identity is illustrated by the following diagrams:

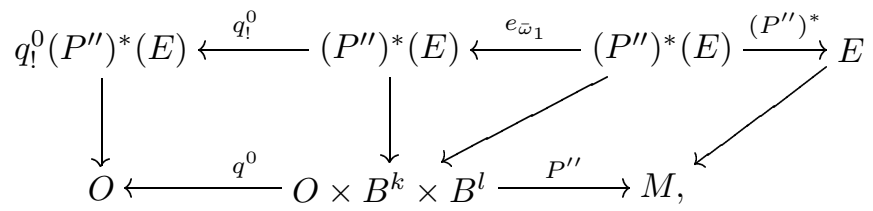




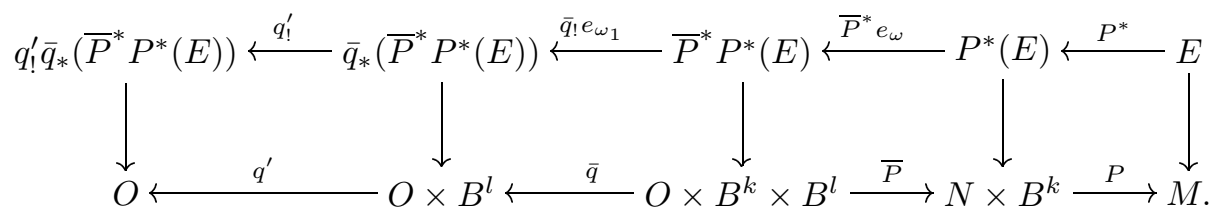

Here the following identities were used:

$$
\begin{gathered}
P^{\prime \prime}(z, s, t)=P\left(P^{\prime}(z, s), t\right)=P \bar{P}(z, s, t), \\
q^{0}=q^{\prime} \bar{q}: O \times B^{k} \times B^{l} \stackrel{\bar{q}}{\rightarrow} O \times B^{l} \stackrel{q^{\prime}}{\rightarrow} O, \\
\tilde{\omega}_{1}=\left(\pi_{l}\right)^{*}\left(\nu_{l}\right) \wedge(\tilde{\pi})^{*}\left(\pi_{k}^{*}\left(\nu_{k}\right)\right)=\omega_{1} \wedge(\tilde{\pi})^{*}(\omega), \\
\tilde{\pi}: O \times B^{k} \times B^{l} \rightarrow O \times B^{k}, \pi_{k}: O \times B^{k} \rightarrow B^{k}, e_{(\tilde{\pi})^{*}(\omega)} \bar{P}^{*}=\bar{P}^{*} e_{\omega} .
\end{gathered}
$$

For the purpose of illustration, we also used an unusual arrow notation for the pullback of vector bundles. On the other hand, $\bar{q}_{!} e_{\omega_{1}} \bar{P}^{*}=e_{\omega_{1}^{\prime}} \bar{q}_{!} \bar{P}^{*}=e_{\omega_{1}^{\prime}}\left(P^{\prime}\right)^{*} q_{\text {! }}$ with $\omega_{1}^{\prime}=\left(\pi^{l}\right)^{*}\left(\nu_{l}\right)$, and $\omega_{1}=\left(\pi_{l}\right)^{*}\left(\nu_{l}\right)$, where $\pi^{l}: O \times B^{l} \rightarrow B^{l}$ and $\pi_{l}: O \times B^{k} \times$ $B^{l} \rightarrow B^{l}$ are the projections. This can be seen from the following diagrams:

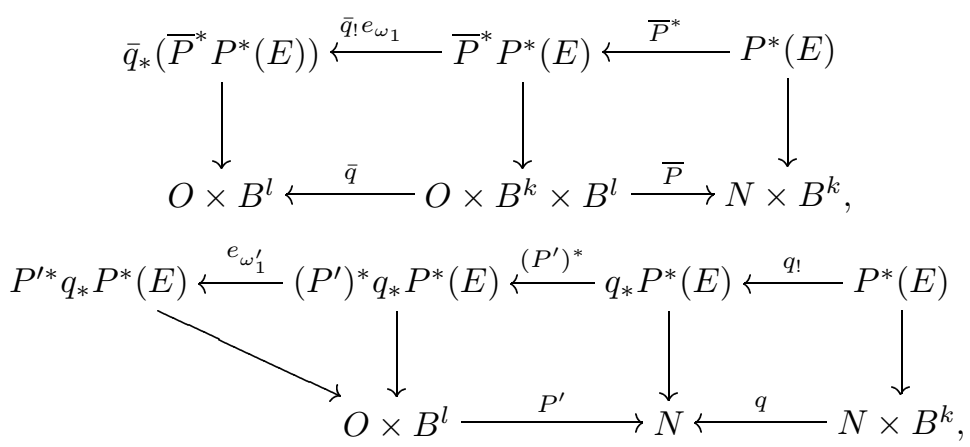

where, as before, $\bar{P}(z, s, t)=\left(P^{\prime}(z, t), s\right)$ and $\bar{q}(z, s, t)=(z, t)$, and $P^{\prime}(z, t)=z$. Therefore,

$$
\begin{aligned}
R_{p^{\prime \prime}, \nu} & =q_{!}^{\prime} \bar{q} ! e_{\omega_{1}} \bar{P}^{*} e_{\omega} P^{*}=q_{!}^{\prime} e_{\omega_{1}^{\prime}}\left(P^{\prime}\right)^{*} q_{!} e_{\omega} P^{*} \\
& =R_{p^{\prime}, \nu_{l}} R_{p, \nu_{k}} .
\end{aligned}
$$

The geometric idea of the first part of the above proof is to throw two small spaces $N \times B^{l}$ and $N \times B^{k}$ into a large space $N \times B^{l} \times B^{k}$, and then to use homotopies between submersions in the large space to compare the maps $R_{p, \nu_{k}}$ and $R_{p^{\prime}, \nu_{l}}$. Similarly, we used the factors $B^{k}$ and $B^{l}$ in the second part of the above proof to break the submersions on the large spaces into submersions on the small spaces. An essential point is that two factors $B^{l}$ and $B^{k}$ in $B^{l} \times B^{k}$ enable us to move around the submersions.

We can now prove Theorem 1 stated in the Introduction.

Proof of Theorem 1. We need only check that $\left(\mathcal{E}_{E}, Q, D_{E}\right)$ and $\left(\mathcal{E}_{h^{*}(E)}, Q, D_{h^{*}(E)}\right)$ satisfy the condition of Proposition 1. By the observation preceding Lemma 9, we can assume that $h$ is smooth. Let $J: M \rightarrow \mathbb{R}^{k}$ be an equivariant embedding $(k \equiv 0(4)), O$ an equivariant tubular neighborhood of $J(M)$ in $\mathbb{R}^{k}$ and $\pi: O \rightarrow M$ the associated equivariant projection (cf. [Bre]). Suppose $J(M)+B^{k} \subset O$. Define a submersion $P: N \times B^{k} \rightarrow M$ by $P(x, t)=\pi(h(x)+t), x \in N, t \in B^{k}$. Let $\nu_{k}$ be 
an equivariant volume form of mass 1 on $B^{k}$ and $\varphi$ be an equivariant smooth map such that $\varphi \nu_{k}=\nu_{k}$. With the notation as in the paragraph preceding Lemma 11, we divide the proof into three steps.

Step 1. Condition (a) of Proposition 1 is valid already since $D^{2}=0$ by the flatness. To check condition (b) of Proposition 1, we define $R$ to be $R_{p, \nu_{k}} \in \mathcal{L}_{G}\left(\mathcal{E}_{E}, \mathcal{E}_{h^{*}(E)}\right)$, i.e., $R=q_{!, \varphi} e_{\omega} P^{*}$. Note that $P_{0}(x)=P(x, o)=\pi(h(x))=h(x)$. Clearly, $q_{!, \varphi}, e_{\omega}$ and $P^{*}$ preserve the domain of $D$ and commute with $D$. In fact, since $P$ is equivariantly homotopic to $h q$,

$$
\begin{aligned}
\left(e_{\varphi} q^{*}\right)^{\prime} e_{\omega} P^{*} \nabla & =\left(e_{\varphi} q^{*}\right)^{\prime} e_{\omega} P^{*}(\nabla) P^{*}=\left(e_{\varphi} q^{*}\right)^{\prime} P^{*}(\nabla) e_{\omega} P^{*} \\
& =h^{*}(\nabla)\left(e_{\varphi} q^{*}\right)^{\prime} e_{\omega} P^{*} .
\end{aligned}
$$

Hence, $R(\operatorname{dom}(D)) \subset \operatorname{dom}(D)$ and $R D_{E}=D_{h^{*}(E)} R$.

Let $\varepsilon \in \mathcal{L}_{G}\left(\mathcal{E}_{E}\right)$ be defined by $\varepsilon(\xi)=(-1)^{\partial \xi} \xi$. Then $\varepsilon^{2}=I, \varepsilon^{\prime}=\varepsilon, \varepsilon D=$ $-D \varepsilon, \varepsilon P^{*}=P^{*} \varepsilon, \varepsilon e_{\omega}=(-1)^{k} e_{\omega} \varepsilon$ and $\varepsilon\left(e_{\varphi} h\right)^{\prime}=(-1)^{k}\left(e_{\varphi} h^{*}\right)^{\prime} \varepsilon$. Hence we get $\varepsilon\left(I-R^{\prime} R\right)=\left(I-R^{\prime} R\right) \varepsilon$. This verifies condition $(e)$ of Proposition 1 .

Step 2. We use Lemma 11 to verify condition $(c)$ of Proposition 1, i.e., $R$ : $\frac{\operatorname{Ker}\left(D_{E}\right)}{i m\left(D_{E}\right)} \rightarrow \frac{\operatorname{Ker}\left(D_{h^{*}(E)}\right)}{i m\left(D_{h^{*}(E)}\right)}$ is an isomorphism. By Lemma $11(a)$, the map $R=R_{p, \nu}$ from $\frac{\operatorname{Ker}\left(D_{E}\right)}{i m\left(D_{E}\right)}$ to $\frac{\operatorname{Ker}\left(D_{h^{*}(E)}\right)}{\operatorname{im}\left(D_{h^{*}(E)}\right)}$ is independent of the submersion $P$ and the volume form $\nu$, i.e., $R_{p, \nu}=R_{p^{\prime}, \nu^{\prime}}$ on $\frac{\operatorname{Ker}\left(D_{E}\right)}{i m\left(D_{E}\right)}$ as long as $P^{\prime}(*, o)$ is equivariantly homotopic to $P(*, o)$. Let $h^{\prime}: M \rightarrow N$ be the smooth map such that $h h^{\prime}$ and $h^{\prime} h$ are (non-equivariantly) homotopic to $I_{M}$ and $I_{N}$, resp. As we construct the submersion $P: N \times B^{k} \rightarrow M$ from $h$, we can find a submersion $P^{\prime}: M \times B^{l} \rightarrow N$ from $h^{\prime}$, namely, $P^{\prime}(x, t)=\pi^{\prime}\left(h^{\prime}(x)+t\right)$ and $\pi^{\prime}: O^{\prime} \rightarrow N$ is a projection with $O^{\prime}$ a tubular neighborhood of $J^{\prime}(N) \subset \mathbb{R}^{l}$ for an equivariant embedding $J^{\prime}$ of $N$ into $\mathbb{R}^{l}$. Then define $P^{\prime \prime}: M \times B^{l} \times B^{k} \stackrel{P^{\prime}}{\rightarrow} N \times B^{k} \stackrel{P}{\rightarrow} M$ by $P^{\prime \prime}(x, s, t)=P\left(P^{\prime}(x, s), t\right)$. Clearly, $P^{\prime \prime}(x, o, o)=P\left(h^{\prime}(x), o\right)=\pi\left(h\left(h^{\prime}(x)\right)+o\right)=h\left(h^{\prime}(x)\right) \stackrel{\text { homotopic }}{\rightarrow} I_{M}(x)$. Hence $R_{p^{\prime \prime}, \nu^{\prime \prime}}=I$ on $\frac{\operatorname{Ker}\left(D_{E}\right)}{i m\left(D_{E}\right)}$ by a non-equivariant version of Lemma $11(a)$. Furthermore, using a non-equivariant counterpart of Lemma $11(b)$, we obtain $R_{p^{\prime}, \nu^{\prime}} R_{p, \nu}=$ $R_{p^{\prime \prime}, \nu^{\prime \prime}}=I$ on $\frac{\operatorname{Ker}\left(D_{E}\right)}{\operatorname{im}\left(D_{E}\right)}$. This proves that $R_{p, \nu}$ is injective. The same reasoning shows, switching the role of $R_{p^{\prime}, \nu^{\prime}}$ and $R_{p, \nu}$, that $R_{p, \nu}$ is surjective in $\frac{\operatorname{Ker}\left(D_{E}\right)}{\operatorname{im}\left(D_{E}\right)}$. Therefore, $R=R_{p, \nu}$ is an isomorphism. Note that this step does not require the equivariance of the above maps.

Step 3. We now check condition $(d)$ of Proposition 1, i.e., there is an equivariant $S \in \mathcal{L}_{G}\left(\mathcal{E}_{E}\right)$ such that $S\left(\operatorname{dom}\left(D_{E}\right)\right) \subset \operatorname{dom}\left(D_{E}\right)$ and $I-R^{\prime} R=D_{E} S+S D_{E}$.

Let $q_{i}: N \times B^{k} \times B^{k} \rightarrow N \times B^{k}$ be the projections, $q_{i}\left(x, t_{1}, t_{2}\right)=\left(x, t_{i}\right), i=1,2$. Also let $q: N \times B^{k} \rightarrow N$ and $\pi_{k}: N \times B^{k} \rightarrow B^{k}$ be the projections, and let $\omega=\pi_{k}^{*}\left(\nu_{k}\right)$. To find the conjugate of $e_{q_{1}^{*}(\omega)} q_{2}^{*}$, for $\tilde{\eta}=\alpha d x_{I} \wedge d t_{J_{2}} \otimes \eta$ and $\tilde{\xi}=\beta d x_{I_{1}} \wedge d t_{J_{1}} \wedge d t_{J_{2}^{\prime}} \otimes \xi$ we compute

$$
Q\left(\tilde{\xi},\left(e_{q_{1}^{*}(\omega)} q_{2}^{*}\right)(\tilde{\eta})\right)=i^{k\left(\left|I_{1}\right|+\left|J_{2}^{\prime}\right|\right)} Q\left(\left(e_{q_{1}^{*}(\omega)} q_{2}^{*}\right)^{\prime}(\tilde{\xi}), \tilde{\eta}\right)
$$

with

$$
\left(e_{q_{1}^{*}(\omega)} q_{2}^{*}\right)^{\prime}(\tilde{\xi})=i^{k\left(\left|I_{1}\right|+\left|J_{2}^{\prime}\right|\right)}\left(\int_{B^{k}} \beta \overline{q_{1}^{*}(\omega)}\right) d x_{I_{1}} \wedge d t_{J_{2}^{\prime}}
$$


for $\left|J_{1}\right|=0$, and 0 otherwise. In particular, if $\tilde{\xi}=e_{q_{2}^{*}(\omega)} q_{1}^{*}(\tilde{\eta})=\alpha\left(q_{1}\right) d x_{I_{1}} \wedge d t_{J_{1}} \wedge$ $q_{2}^{*}(\omega)$, then

$$
\begin{aligned}
& \left(e_{q_{1}^{*}(\omega)} q_{2}^{*}\right)^{\prime}\left(e_{q_{2}^{*}(\omega)} q_{1}^{*}\right)\left(\alpha d x_{I} \wedge d t_{J_{1}} \otimes \eta\right) \\
& \quad=i^{k\left(\left|I_{1}\right|+k\right)} \int_{B^{k}} \alpha\left(q_{1}\right) \overline{q_{1}^{*}(\omega)} d x_{I_{1}} \wedge q_{2}^{*}(\omega) \otimes \eta \\
& \quad=i^{k\left|I_{1}\right|+k^{2}}\left(\int_{B^{k}} \alpha\left(q_{1}\right) \overline{q_{1}^{*}(\omega)}\right) d x_{I_{1}} \wedge q_{2}^{*}(\omega) \otimes \eta, \quad\left|J_{1}\right|=0,
\end{aligned}
$$

and it is zero for $\left|J_{1}\right| \neq 0$. On the other hand,

$$
\begin{aligned}
\left(q_{!} e_{\omega}\right)\left(\alpha d x_{I_{1}} \wedge d t_{J_{1}}\right) \otimes \eta & =q_{!}\left(\alpha d x_{I_{1}} \wedge d t_{J_{1}} \wedge \omega \otimes \eta\right) \\
& =i^{k\left(n-\left|I_{1}\right|\right)}\left(\int_{B^{k}} \varphi \alpha \omega\right) d x_{I_{1}} \otimes \eta
\end{aligned}
$$

If $\tilde{\xi}=\beta d x_{I_{1}^{\prime}} \otimes \xi$, then

$$
\begin{aligned}
& Q\left(\tilde{\xi}, q ! e_{\omega}\left(\alpha d x_{I_{1}} \wedge d t_{J_{1}}\right)\right) \\
& \quad=i^{-k n+2 k\left|I_{1}^{\prime}\right|} i^{\left(\left|I_{1}^{\prime}\right|+k\right)\left(n+k-\left|I_{1}^{\prime}\right|-k\right)} \int_{N \times B^{k}}\left(\bar{\beta} \varphi \omega \wedge d x_{I_{1}^{\prime}}\right) \wedge \alpha d x_{I_{1}} \otimes\langle\xi, \eta\rangle \\
& \quad=i^{-k n+2 k\left|I_{1}^{\prime}\right|} Q\left(\left(q ! e_{\omega}\right)^{\prime}(\tilde{\xi}), \tilde{\eta}\right) .
\end{aligned}
$$

Consequently, $\left(q_{!} e_{\omega}\right)^{\prime}(\tilde{\xi})=i^{-k n+2 k\left|I_{1}^{\prime}\right|} \beta \omega \wedge d x_{I_{1}^{\prime}} \otimes \xi$. Using this identity, we have

$$
\begin{aligned}
\left(q ! e_{\omega}\right)^{\prime}\left(q ! e_{\omega}\right)\left(\alpha d x_{I_{1}} \otimes \eta\right) & =i^{-k n+2 k\left|I_{1}^{\prime}\right|} i^{k\left(n-\left|I_{1}\right|\right)}\left(\int_{B^{k}} \alpha \omega\right) \omega \wedge d x_{I_{1}} \otimes \eta \\
& =i^{-k\left|I_{1}\right|}\left(\int_{B^{k}} \alpha \omega\right) d x_{I_{1}} \wedge \omega \otimes \eta .
\end{aligned}
$$

Comparing this with (6), we get

(6) $\left(q ! e_{\omega}\right)^{\prime}\left(q_{!} e_{\omega}\right)\left(\alpha d x_{I_{1}} \otimes \eta\right)=i^{-2 k\left|I_{1}\right|-k^{2}}\left(e_{q_{1}^{*}(\omega)} q_{2}^{*}\right)^{\prime}\left(e_{q_{2}^{*}(\omega)} q_{1}^{*}\right)\left(\alpha d x_{I_{1}} \otimes \eta\right)$.

Furthermore,

$$
\begin{aligned}
\left(e_{q_{2}^{*}(\omega)} q_{2}^{*}\right)\left(\alpha d x_{I} \wedge d t_{J_{2}} \otimes \eta\right) & =\alpha\left(q_{2}\right) d x_{I} \wedge d t_{J_{2}} \wedge q_{2}^{*}(\omega) \otimes \eta \\
& =\alpha d x_{I} \wedge q_{2}^{*}(\omega) \otimes \eta,\left|J_{2}\right|=0
\end{aligned}
$$

and

$$
\begin{aligned}
\left(e_{q_{1}^{*}(\omega)} q_{2}^{*}\right)^{\prime}\left(e_{q_{2}^{*}(\omega)} q_{2}^{*}\right)(\tilde{\eta}) & =i^{k(|I|+k)}\left(\int_{B^{k}} \alpha \overline{q_{1}^{*}(\omega)}\right) d x_{I} \wedge q_{2}^{*}(\omega) \otimes \eta \\
& =i^{k^{2}+k|I|}\left(\int_{B^{k}} \overline{q_{1}^{*}(\omega)}\right) \alpha d x_{I} \wedge q_{2}^{*}(\omega) \otimes \eta .
\end{aligned}
$$

Clearly, $e_{\omega}\left(\alpha d x_{I} \otimes \eta\right)=\alpha d x_{I} \wedge \omega \otimes \eta$. Therefore,

$$
e_{\omega}=i^{-k^{2}-k|I|}\left(e_{q_{1}^{*}(\omega)} q_{2}^{*}\right)^{\prime}\left(e_{q_{2}^{*}(\omega)} q_{2}^{*}\right)
$$

This together with (6) proves that $e_{\omega}=i^{k|I|}\left(q ! e_{\omega}\right)^{\prime}\left(q_{!} e_{\omega}\right)$ modulo the boundary $D_{E} S+S D_{E}$ by Lemma 10 . This implies that

$$
R^{\prime} R=\left(P^{*}\right)^{\prime}\left(q ! e_{\omega}\right)^{\prime}\left(q ! e_{\omega}\right) P^{*}=P_{!}\left(q ! e_{\omega}\right)^{\prime}\left(q ! e_{\omega}\right) P^{*}=P_{!} e_{\omega} P^{*}
$$

modulo the boundary $D_{E} S+S D_{E}$. Hence it suffices to show that $P_{!} e_{\omega} P^{*}=I$ modulo the boundary $D_{E} S+S D_{E}$. 
Let $P_{1}: M \times B^{k} \rightarrow M, P_{2}: M \times B^{k} \rightarrow B^{k}$ be the projections and $\tilde{\pi}: M \times B^{k} \rightarrow$ $M$ be the equivariant submersion given by $\tilde{\pi}(x, t)=\pi(x+t)$ and $\omega^{\prime}=P_{2}^{*}\left(\nu_{k}\right)$. Let $P: N \times B^{k} \rightarrow M$ be the equivariant submersion, $P(x, t)=\pi(h(x)+t)$.

(a) $\left(P_{1}\right) ! e_{\omega^{\prime}} P_{1}^{*}$ is the identity:

$$
\begin{aligned}
\left(P_{1}\right) ! e_{\omega^{\prime}} P_{1}^{*}\left(\alpha d x_{I} \otimes \eta\right) & =\left(P_{1}\right) ! e_{\omega^{\prime}} \alpha\left(P_{1}\right) d x_{I} \otimes \eta=\left(P_{1}\right) !, \varphi\left(\alpha\left(P_{1}\right) d x_{I} \wedge \omega^{\prime} \otimes \eta\right) \\
& =i^{(n-|I|) k}\left(\int \varphi \omega^{\prime}\right) \alpha d x_{I} \otimes \eta=\alpha d x_{I} \otimes \eta, k \equiv 0(4)
\end{aligned}
$$

(b) $P_{!} e_{\omega} P^{*}=\tilde{\pi}_{!} e_{\omega^{\prime}} \tilde{\pi}^{*}:$

$$
\begin{aligned}
P_{!} e_{\omega} P^{*} & \left(\alpha d x_{I} \otimes \eta\right)=P_{!}\left(\alpha(P) d P_{I} \wedge \omega \otimes \eta\right) \\
& =i^{(n-|I|) k} \int_{N \times B^{k}} \alpha(P) \omega d P_{I} \otimes \eta \\
& \left.=i^{k(n-|I|)} \int_{N \times B^{k}} \alpha(\pi(h(x)+t))\right) \nu_{k}\left(\pi_{k}(x, t)\right) d(\pi(h(x)+t))_{I} \otimes \eta \\
& =i^{k(n-|I|)} \int_{M \times B^{k}} \alpha(\pi(y+t)) \nu_{k}(t) d \pi_{I} \otimes \eta \\
& =\tilde{\pi}_{!}\left(\alpha(\tilde{\pi}) d \pi_{I} \omega^{\prime} \otimes \eta\right)=\left(\tilde{\pi}_{!} e_{\omega^{\prime}} \tilde{\pi}^{*}\right)\left(\alpha d x_{I} \otimes \eta\right) .
\end{aligned}
$$

Finally, $\tilde{\pi}_{!} e_{\omega^{\prime}} \tilde{\pi}^{*}=\left(P_{1}\right) ! e_{\omega^{\prime}} P_{1}^{*}$ modulo the boundary $D_{E} S+S D_{E}$ by Lemma 11 . Therefore, $R^{\prime} R=I$ modulo the boundary $D_{E} S+S D_{E}$.

Theorem 1 will play a crucial role in proving the equivariant Novikov conjecture for groups acting on Euclidean buildings. It provides a substitute for the equivariant Mishchenko symmetric signature, as we pointed out earlier.

\section{Equivariant Connes-Gromov-Moscovici Theorem}

Let $\mathcal{A}$ be a $G$ - $C^{*}$-algebra, $E_{0}$ a finitely generated projective Hilbert $G$ - $\mathcal{A}$-module, and $M$ a closed, oriented Riemannian $G$-manifold. Let $E$ be a $G$ - $\mathcal{A}$-bundle over $M$ whose fiber is $E_{0}$. Suppose $\nabla$ is a unitary connection of $E$ and $\Theta=\nabla^{2}$ is its curvature. Let $\tau>0$. $\nabla$ is said to be $\tau$-flat if

$$
\|\Theta\|=\max _{x \in M}\left\{\left\|\Theta_{x}\right\|\right\}<\tau
$$

where $\left\|\Theta_{x}\right\|$ is the norm of the operator $\Theta_{x}$ on $\wedge_{\mathbb{C}}^{2} T_{x} M \otimes E_{x}$,

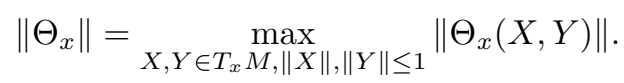

The norm of $\|\Theta\|$ is equal to the norm of $\Theta$ as an operator on the Hilbert $G$ - $\mathcal{A}$ module $\mathcal{E}_{E} . \quad E$ is called almost flat if for every $\tau>0$ there is a $\tau$-flat unitary connection on $E$. An example of $\tau$-flat bundles will be given at the end of this section.

To prove our main theorem of this section, we need a proposition which is the generalization of Proposition 1. We first have the following lemma.

Lemma 12. (a) Let $\tau, k>0$ be such that $6 \sqrt{2} \tau k^{2}<1$. Suppose that $U$ is an equivariant regular operator on a Hilbert $G$-A-module $\mathcal{E}$ such that $i m(U) \subset \operatorname{dom}(U)$ and $\left\|U^{2}\right\|<\tau^{2}$. If there are equivariant $R, S \in \mathcal{L}_{G}(\mathcal{E}), S$ invertible, such that 
$R(\operatorname{dom}(U)) \subset \operatorname{dom}(U), R U+U R=S,\|R\| \leq k$ and $\left\|S^{-1}\right\| \leq k$, then $U+U^{*}$ has a bounded inverse with the norm

$$
\left\|\left(U+U^{*}\right)^{-1}\right\| \leq \frac{2 k^{2}}{1-6 \sqrt{2} \tau k^{2}} .
$$

(b) Let $\tau, k>0$ be such that $2 \sqrt{2} \tau k^{4}(k+3 \sqrt{k})<1$. Suppose $(\mathcal{E}, Q, D) \in \mathcal{L}_{G, u}^{0}(\mathcal{A})$, and $T \in \mathcal{L}_{G}(\mathcal{E})$ is invertible and equivariant such that $Q(\xi, \eta)=\langle\xi, T \eta\rangle$ for the scalar product $\langle.,$.$\rangle on \mathcal{E}$. If $\left\|D^{2}\right\| \leq \tau^{2},\left\|D^{\prime}+D\right\| \leq \tau$ and there are $R, S \in \mathcal{L}_{G}(\mathcal{E})$ equivariant, with $S$ invertible, such that $R(\operatorname{dom}(D)) \subset \operatorname{dom}(D)$ and $D R \in \mathcal{L}_{G}(\mathcal{E})$, $D R+R D=S,\|R\| \leq k,\left\|S^{-1}\right\| \leq k$ and $\|T\|\left\|T^{-1}\right\| \leq k^{2}$, then $\psi_{0, u}((\mathcal{E}, Q, D))=0$.

Proof. We first prove part $(a)$. Let

$$
U_{1}=\left[\begin{array}{cc}
U & U^{2} / \tau \\
-\tau & -U
\end{array}\right], \quad R_{1}=\left[\begin{array}{cc}
R & 0 \\
0 & -R
\end{array}\right], \quad S_{1}=\left[\begin{array}{cc}
S & 0 \\
0 & S
\end{array}\right] .
$$

Then $U_{1}^{2}=0$, and

$$
R_{1} U_{1}+U_{1} R_{1}-S_{1}=\left[\begin{array}{cc}
0 & \left(R U^{2}-U^{2} R\right) / \tau \\
0 & 0
\end{array}\right]
$$

Since

$$
\left\|R_{1} U_{1}+U_{1} R_{1}-S_{1}\right\|=\left\|\left(R U^{2}-U^{2} R\right) / \tau\right\| \leq \frac{2}{\tau}\|R\|\left\|U^{2}\right\| \leq 2 k \tau<1,
$$

and

$$
\begin{aligned}
\left\|S_{1}^{-1}\left(R_{1} U_{1}+U_{1} R_{1}-S_{1}\right)\right\| & \leq\left\|S_{1}^{-1}\right\|\left\|\left(R_{1} U_{1}+U_{1} R_{1}-S_{1}\right)\right\| \\
& \leq \sqrt{2} k(2 k \tau)=2 \sqrt{2} k^{2} \tau<1,
\end{aligned}
$$

it follows that $R_{1} U_{1}+U_{1} R_{1}$ has the inverse

$$
\left(R_{1} U_{1}+U_{1} R_{1}\right)^{-1}=\left(I+S_{1}^{-1}\left(R_{1} U_{1}+U_{1} R_{1}-S_{1}\right)\right)^{-1} S_{1}^{-1},
$$

and

$\left\|\left(R_{1} U_{1}+U_{1} R_{1}\right)^{-1}\right\| \leq\left\|\left(I+S_{1}^{-1}\left(R_{1} U_{1}+U_{1} R_{1}-S_{1}\right)\right)^{-1} S_{1}^{-1}\right\| \leq \frac{1}{1-2 \sqrt{2} k^{2} \tau} \sqrt{2} k$.

Now

$$
U_{1}^{*}=\left[\begin{array}{cc}
U^{*} & -\tau \\
U^{2 *} / \tau & -U^{*}
\end{array}\right], \quad U_{1}+U_{1}^{*}=\left[\begin{array}{cc}
U+U^{*} & U^{2} / \tau-\tau \\
-\tau+U^{*} / \tau & -\left(U+U^{*}\right)
\end{array}\right] .
$$

We show that $U_{1}+U_{1}^{*}$ is invertible and $\left\|\left(U_{1}+U_{1}^{*}\right)^{-1}\right\| \leq \frac{2 k^{2}}{1-2 \sqrt{2} k^{2} \tau}$. In fact, $U_{1}$ commutes with $U_{1} R_{1}+R_{1} U_{1}$ since $U_{1}^{2}=0$. Let $W=\left(R_{1} U_{1}+U_{1} R_{1}\right)^{-1}$. Then $U_{1}$ also commutes with $W$, and $U_{1} W R_{1}+W R_{1} U_{1}=W\left(R_{1} U_{1}+U_{1} R_{1}\right)=I$. Since

$$
U_{1} R_{1}=\left(R_{1} U_{1}+U_{1} R_{1}\right) W U_{1} R_{1}=U_{1} R_{1} W U_{1} R_{1}
$$

and

$$
R_{1} U_{1}=R_{1} U_{1} W\left(R_{1} U_{1}+U_{1} R_{1}\right)=R_{1} U_{1} W R_{1} U_{1}
$$

it follows that $W U_{1} R_{1}=W U_{1} R_{1} W U_{1} R_{1}$ and $W R_{1} U_{1}=W R_{1} U_{1} W R_{1} U_{1}$, i.e., $W U_{1} R_{1}$ and $W R_{1} U_{1}$ are idempotents. Thus, we can find an equivariant projection $P$ such that $P\left(W U_{1} R_{1}\right)=P$ and $\left(W U_{1} R_{1}\right) P=W U_{1} R_{1}$. By Remark $2,\left(U_{1}+U_{1}^{*}\right)$ has the inverse $\left(U_{1}+U_{1}^{*}\right)^{-1}=P\left(W R_{1}\right)(I-P)+(I-P)\left(W R_{1}\right)^{*} P$. Hence,

$$
\left\|\left(U_{1}+U_{1}^{*}\right)^{-1}\right\| \leq\left\|W R_{1}\right\| \leq \frac{\sqrt{2} k}{1-2 \sqrt{2} k^{2} \tau} \sqrt{2} k=\frac{2 k^{2}}{1-2 \sqrt{2} k^{2} \tau} \stackrel{\text { def }}{=} k_{1} .
$$

From this estimate, setting 


$$
Z \stackrel{\text { def }}{=}(U \oplus(-U))+(U \oplus(-U))^{*}-\left(U_{1}+U_{1}^{*}\right)=\left[\begin{array}{cc}
0 & \tau-U^{2} / \tau \\
\tau-U^{2} / \tau & 0
\end{array}\right],
$$

we get $\|Z\| \leq \sqrt{2}\left\|\tau-U^{2} / \tau\right\| \leq 2 \sqrt{2} \tau$ and

$$
\begin{aligned}
\left((U \oplus(-U))+(U \oplus(-U))^{*}\right)^{-1} & =\left(Z+\left(U_{1}+U_{1}^{*}\right)\right)^{-1} \\
& \left.=\left(I+\left(\left(U_{1}+U_{1}^{*}\right)\right)^{-1} Z\right)^{-1}\left(U_{1}+U_{1}^{*}\right)\right)^{-1},
\end{aligned}
$$

since $\left\|\left(U_{1}+U_{1}^{*}\right)^{-1} Z\right\| \leq \frac{2 k^{2}}{1-2 \sqrt{2} k^{2} \tau} 2 \sqrt{2} \tau<1$, i.e., $6 \sqrt{2} \tau k^{2}<1$. Then

$$
\begin{aligned}
\|((U & \left.\oplus(-U))+(U \oplus(-U))^{*}\right)^{-1} \| \\
& \left.\leq\left\|\left(I+\left(\left(U_{1}+U_{1}^{*}\right)\right)^{-1} Z\right)^{-1}\right\| \|\left(U_{1}+U_{1}^{*}\right)\right)^{-1} \| \\
& \leq \frac{1}{1-\frac{2 k^{2}}{1-2 \sqrt{2} k^{2} \tau} 2 \sqrt{2} \tau} \frac{2 k^{2}}{1-2 \sqrt{2} k^{2} \tau}=\frac{2 k^{2}}{1-6 \sqrt{2} \tau k^{2}} .
\end{aligned}
$$

We now prove part (2). Define a new scalar product on $\mathcal{E}$ by $(\xi, \eta)=\langle\xi,|T| \eta\rangle$. If $Z \in \mathcal{L}(\mathcal{E})$, then

$$
\begin{aligned}
\|Z\|_{(.)}^{2} & =\max _{\|\xi\|_{(.)} \leq 1}\|(Z \xi, Z \xi)\| \\
& =\max _{\left\||T|^{\frac{1}{2}} \xi\right\| \leq 1}\left\|\left\langle Z|T|^{-\frac{1}{2}}\left(|T|^{\frac{1}{2}} \xi\right),|T| Z|T|^{-\frac{1}{2}}\left(|T|^{\frac{1}{2}} \xi\right)\right\rangle\right\| \\
& \leq \max _{\left\||T|^{\frac{1}{2}} \xi\right\| \leq 1}\left\|\left.T\left|\|\| Z\left\|^{2}\right\|\right| T\right|^{-\frac{1}{2}}\right\|^{2}\left\||T|^{\frac{1}{2}} \xi\right\|^{2} \\
& =\|Z\|^{2}\left\||T|^{-\frac{1}{2}}\right\|^{2}\||T|\|,
\end{aligned}
$$

namely, $\|Z\|_{(.)} \leq k\|Z\|$. With the scalar product (.) on $\mathcal{E}$, we have $\left\|D^{2}\right\|_{(.)} \leq$ $k\|D\| \leq k \tau^{2},\left\|D^{\prime}+D\right\|_{(.)} \leq k\left\|D^{\prime}+D\right\| \leq k \tau,\|R\|_{(.)} \leq k\|R\| \leq k^{2}$ and $\|S\|_{(.)} \leq$ $k\|S\| \leq k^{2}$. Using part (a) with $k$ and $\tau$ replaced by $k^{2}$ and $\sqrt{k} \tau$, we obtain that $D+D^{*}$ is invertible and $\left\|\left(D+D^{*}\right)^{-1}\right\|_{(.)} \leq \frac{2\left(k^{2}\right)^{2}}{1-6 \sqrt{2} \sqrt{k} \tau\left(k^{2}\right)^{2}}$, since $6 \sqrt{2} \sqrt{k} \tau\left(k^{2}\right)^{2}=$ $6 \sqrt{2} \tau \sqrt{k} k^{4}<1$ by assumption. Thus we are done for $D^{\prime}=-D$.

Note that $(\xi, \eta)=\langle\xi,|T| \eta\rangle=Q\left(\xi, T^{-1}|T| \eta\right)$. Let $T_{1}=T^{-1}|T|, T_{1}^{2}=I . T_{1}$ is compatible with $Q$. Let $P_{1}=\frac{I+T_{1}}{2}, P_{1}^{2}=P_{1}=P_{1} T_{1}$ and $\left(I-P_{1}\right) T_{1}=T_{1}-P_{1}=$ $-\left(I-P_{1}\right)$. Then

$$
\begin{aligned}
\left(D+D^{*}\right)^{(0)} & =P_{1}\left(D+D^{*}\right) P_{1}+\left(I-P_{1}\right)\left(D+D^{*}\right)\left(I-P_{1}\right) \\
& =P_{1}\left(D+D^{\prime}\right) P_{1}+\left(I-P_{1}\right)\left(D+D^{\prime}\right)\left(I-P_{1}\right) .
\end{aligned}
$$

This yields $\left\|\left(D+D^{*}\right)^{(0)}\right\|_{(.)} \leq \sqrt{2}\left\|D+D^{\prime}\right\|_{(.)}$, and then

$$
\begin{aligned}
\|(D & \left.+D^{*}\right)-\left(D+D^{*}\right)^{(1)}\left\|_{(.)}\right\|\left(D+D^{*}\right)^{-1} \|_{(.)} \\
& =\left\|\left(D+D^{*}\right)^{(0)}\right\|_{(.)}\left\|\left(D+D^{*}\right)^{-1}\right\|_{(.)} \\
& \leq \sqrt{2} k \tau \frac{2\left(k^{2}\right)^{2}}{1-6 \sqrt{2} \sqrt{k} \tau\left(k^{2}\right)^{2}}<1
\end{aligned}
$$

since $2 \sqrt{2} \tau k^{4}(k+3 \sqrt{k})<1$. Therefore, $\left(D+D^{*}\right)^{-1}$ is invertible:

$$
\begin{aligned}
\left(\left(D+D^{*}\right)^{(1)}\right)^{-1} & =\left(\left(D+D^{*}\right)-\left(\left(D+D^{*}\right)-\left(D+D^{*}\right)^{(1)}\right)\right)^{-1} \\
& =\left(I-\left(D+D^{*}\right)^{-1}\left(\left(D+D^{*}\right)-\left(D+D^{*}\right)^{(1)}\right)\right)^{-1}\left(D+D^{*}\right)^{-1} .
\end{aligned}
$$


Observe that the map $\psi_{u, 0}$ in Definition 3 can be defined for $D^{\prime} \neq-D$, in which one replaces $\left(D+D^{*}\right)$ by $\left(D+D^{*}\right)^{(1)}$ in the formula for $F$. We have shown that $\left(D+D^{*}\right)^{(1)}$ is invertible. Hence, $\psi_{u, 0}((\mathcal{E}, Q, D))=0$.

The above proof is based on the elementary fact that for a bounded operator $A$ with $\|A\|<1, I+A$ is invertible. Indeed, this is the main reason why Theorem 1 can be extended to the case of almost flat bundles. Once the curvature is small, the norms of relevant operators can be estimated by a small constant. We will also use this fact in the following proposition, which is an extension of Proposition 1 to the almost flat case.

Proposition 2. Let $\left(\mathcal{E}_{i}, Q_{i}, D_{i}\right) \in \mathcal{L}_{G, u}^{0}(\mathcal{A})$ be such that $D_{i}^{\prime}=-D_{i}$ and $\mathcal{E}_{i}$ have the scalar products compatible with $Q_{i}, i=1,2$. Suppose that there are equivariant $R \in \mathcal{L}\left(\mathcal{E}_{1}, \mathcal{E}_{2}\right)$ and $\tau, k>0$ satisfying

(a) $\left\|D_{i}^{2}\right\| \leq \tau^{2},\|R\| \leq k, R\left(\operatorname{dom}\left(D_{1}\right)\right) \subset \operatorname{dom}\left(D_{1}\right)$ and $\left\|R D_{1}-D_{2} R\right\| \leq \tau^{2}$;

(b) there is an equivariant $S \in \mathcal{L}\left(\mathcal{E}_{1}\right)$ with $\|S\| \leq k, S\left(\operatorname{dom}\left(D_{1}\right)\right) \subset \operatorname{dom}\left(D_{1}\right)$, and $\left\|I-R^{\prime} R-D_{1} S-S D_{1}\right\| \leq \tau^{2}$

(c) there is an equivariant $\varepsilon \in \mathcal{L}\left(\mathcal{E}_{1}\right)$ with $\varepsilon\left(\operatorname{dom}\left(D_{1}\right)\right) \subset \operatorname{dom}\left(D_{1}\right), \varepsilon D_{1}=$ $-D_{1} \varepsilon, \varepsilon^{2}=I, \varepsilon^{\prime}=\varepsilon$ and $\varepsilon\left(I-R^{\prime} R\right)=\left(I-R^{\prime} R\right) \varepsilon$;

$(d)$ there is an equivariant $Z \in \mathcal{L}\left(\mathcal{E}_{2}\right)$ with $\|Z\| \leq k, Z\left(\operatorname{dom}\left(D_{2}\right)\right) \subset \operatorname{dom}\left(D_{2}\right)$ and $\left\|I-R R^{\prime}-D_{2} Z-Z D_{2}\right\| \leq \tau^{2}$.

Then for $64 \tau\left(k+4 \sqrt{2} k^{3}(1+k)^{2}\right)^{5}<1, \tau<\frac{1}{2 \sqrt{2} 2^{\frac{1}{4}}}$ and $k \geq 1$,

$$
\psi_{0, u}\left(\left(\mathcal{E}_{1}, Q_{1}, D_{1}\right)\right)=\psi_{0, u}\left(\left(\mathcal{E}_{2}, Q_{2}, D_{2}\right)\right) .
$$

Furthermore, suppose $(d)$ is replaced by the following condition:

$\left(d^{\prime}\right)$ there are equivariant $W \in \mathcal{L}\left(\mathcal{E}_{2}, \mathcal{E}_{1}\right)$ and $Z \in \mathcal{L}_{G}\left(\mathcal{E}_{2}\right)$ such that $\|Z\| \leq k$, $\|W\| \leq k, W\left(\operatorname{dom}\left(D_{2}\right)\right) \subset \operatorname{dom}\left(D_{1}\right), Z\left(\operatorname{dom}\left(D_{2}\right)\right) \subset \operatorname{dom}\left(D_{2}\right),\left\|W D_{2}-D_{1} W\right\| \leq \tau^{2}$ and $\left\|I-R W-D_{2} Z-Z D_{2}\right\| \leq \tau^{2}$.

Then for $64 \tau \sqrt{1+6 k^{2}}\left\{k\left(1+2 k^{2}\right)+4 \sqrt{2} k^{3}\left(1+2 k^{2}\right)^{3}\left(1+k\left(1+2 k^{2}\right)\right)^{2}\right\}^{5}<1$, $\tau<\frac{1}{2 \sqrt{2} 2^{\frac{1}{4}} \sqrt{1+6 k^{2}}}$ and $k \geq 1$,

$$
\psi_{0, u}\left(\left(\mathcal{E}_{1}, Q_{1}, D_{1}\right)\right)=\psi_{0, u}\left(\left(\mathcal{E}_{2}, Q_{2}, D_{2}\right)\right) .
$$

Proof. The proof is divided into four steps.

Step 1. As in the proof of Proposition 1, we can assume $S=-S^{\prime}$ and $Z=-Z^{\prime}$ by considering $\frac{S-S^{\prime}}{2}$ and $\frac{Z-Z^{\prime}}{2}$ that satisfy (b) and (d).

Let $(\mathcal{E}, Q, D)=\left(\mathcal{E}_{1} \oplus \mathcal{E}_{2}, Q_{1} \oplus\left(-Q_{2}\right), D_{1} \oplus\left(-D_{2}\right)\right)$. By Lemma 7 , it suffices to show that $\psi_{0, u}((\mathcal{E}, Q, D))=0$. The proof is similar to that of Proposition 1 .

For $t \in[0,1]$ define

$$
T_{t}=\left[\begin{array}{cc}
I & 0 \\
i t R \varepsilon & I
\end{array}\right], \quad L_{t}=\left[\begin{array}{cc}
I-R^{\prime} R & (i \varepsilon+t S) R^{\prime} \\
R(i \varepsilon+t S) & I
\end{array}\right], \quad \nabla_{t}=\left[\begin{array}{cc}
D_{1} & t R^{\prime} \\
0 & -D_{2}
\end{array}\right] .
$$

These operators are all equivariant. Let $B_{t}(\xi, \eta)=Q\left(T_{t} \xi, T_{t} \eta\right)$ and $C_{t}(\xi, \eta)=$ $Q\left(L_{t} \xi, \eta\right)$. We check that $\left(\mathcal{E}, B_{t}, D\right) \in \mathcal{L}_{G, u}^{0}(\mathcal{A})$.

If $D_{t}^{\prime}$ denotes the conjugate of $D$ with respect to $B_{t}$, then, as before, $D_{t}^{\prime}=$ $\left(T_{t}^{\prime} T_{t}\right)^{-1} D^{\prime}\left(T_{t}^{\prime} T_{t}\right)=-\left(T_{t}^{\prime} T_{t}\right)^{-1} D\left(T_{t}^{\prime} T_{t}\right)$. Since

$$
T_{t} D-D T_{t}=\left[\begin{array}{cc}
0 & 0 \\
-i t\left(R D_{1}-D_{2} R\right) \varepsilon & 0
\end{array}\right]
$$


$T_{t} D-D T_{t}$ is in $\mathcal{L}_{G}(\mathcal{E})$ by condition $(a)$. This implies that $D+D_{t}^{\prime} \in \mathcal{L}_{G}(\mathcal{E})$, since $D+D_{t}^{\prime}=\left(T_{t}^{\prime} T_{t}\right)^{-1}\left(T_{t}^{\prime}\left(T_{t} D-D T_{t}\right)+\left(T_{t}^{\prime} D-D T_{t}^{\prime}\right) T_{t}\right)$. Now

$$
\begin{gathered}
\left\|\left[T_{t}, D\right]\right\| \leq t\left\|R D_{1}-D_{2} R\right\| \leq t \tau^{2}, \\
\left\|\left[T_{t}^{\prime}, D\right]\right\| \leq t\left\|R^{\prime} D_{2}-D_{1} R^{\prime}\right\| \leq t \tau^{2},
\end{gathered}
$$

and $\left\|T_{t}\right\| \leq(\|R\| t+1) \sqrt{2} \leq \sqrt{2}(1+t k),\left\|T_{t}^{\prime}\right\| \leq \sqrt{2}(1+t k)$. Here we used the norm $\|(x, y)\|=\sqrt{\|x\|^{2}+\|y\|^{2}}$ on $\mathcal{E}=\mathcal{E}_{1} \oplus \mathcal{E}_{2}$. Clearly,

$$
\begin{gathered}
T_{t}^{-1}=\left[\begin{array}{cc}
I & 0 \\
-i t R \varepsilon & I
\end{array}\right], \quad\left(T_{t}^{\prime}\right)^{-1}=\left[\begin{array}{cc}
I & -i t \varepsilon R^{\prime} \\
0 & I
\end{array}\right], \\
\left(T_{t}^{\prime} T_{t}\right)^{-1}=\left[\begin{array}{cc}
I & -i t \varepsilon R^{\prime} \\
-i t R \varepsilon & t^{2} R R^{\prime}+I
\end{array}\right] .
\end{gathered}
$$

We have $\left\|\left(T_{t}^{\prime} T_{t}\right)^{-1}\right\| \leq \sqrt{2}\left(1+t^{2}\left\|R R^{\prime}\right\|+t\|R\|\right) \leq \sqrt{2}\left(1+t k^{2}+t k\right)$, and

$$
\begin{aligned}
\left\|D_{1}+D_{t}^{\prime}\right\| & \leq\left\|\left(T_{t}^{\prime} T_{t}\right)^{-1}\right\|\left(\left\|T_{t}\right\|\left\|\left[T_{t}, D\right]\right\|+\left\|\left[T_{t}^{\prime}, D\right]\right\|\left\|T_{t}\right\|\right) \\
& \leq 4 t \tau^{2}(1+t k)\left(1+t^{2} k+t k\right) .
\end{aligned}
$$

This proves $D+D_{t}^{\prime} \in \mathcal{L}_{G}(\mathcal{E})$. Obviously, $D^{2} \in \mathcal{L}_{G}(\mathcal{E})$, and, as in the proof of Proposition 1, we can find equivariant $R_{1}$ and $S_{1} \in \mathcal{L}_{G}(\mathcal{E})$ such that $D S_{1}+R_{1} D-I \in$ $\mathcal{K}_{G}(\mathcal{E})$. We have thus verified that $\left(\mathcal{E}, B_{t}, D\right) \in \mathcal{L}_{G, u}^{0}(\mathcal{A})$.

Step 2. We now check that $\left(\mathcal{E}, C_{t}, \nabla_{t}\right) \in \mathcal{L}_{G, u}^{0}(\mathcal{A})$ for $t$ near 0 . Since $L_{0}=T_{1}^{\prime} T_{1}$,

$$
\left\|L_{0}^{-1}\right\| \leq\left\|T_{1}^{-1}\right\|\left\|\left(T_{1}^{\prime}\right)^{-1}\right\| \leq(\sqrt{2}(1+k))^{2}=2(1+k)^{2} .
$$

Note that

$$
L_{t}=\left[\begin{array}{cc}
I-R^{\prime} R & i \varepsilon R^{\prime} \\
i R \varepsilon & I
\end{array}\right]+\left[\begin{array}{cc}
0 & t S R^{\prime} \\
t R S & 0
\end{array}\right]=L_{0}\left(I+L_{0}^{-1}\left[\begin{array}{cc}
0 & t S R^{\prime} \\
t R S & 0
\end{array}\right]\right) .
$$

We see that if

$$
\left\|L_{0}^{-1}\left[\begin{array}{cc}
0 & t S R^{\prime} \\
t R S & 0
\end{array}\right]\right\| \leq 2(1+k)^{2} \sqrt{2}\|S\|\|R\| t \leq 2 \sqrt{2}(1+k)^{2} k^{2} t<1
$$

then $L_{t}$ is invertible. Let $t_{0}=\frac{1}{4 \sqrt{2}(1+k)^{2} k^{2}}$. We have

$$
\left\|L_{t}^{-1}\right\| \leq\left\|\left(I+L_{0}^{-1}\left[\begin{array}{cc}
0 & t S R^{\prime} \\
t R S & 0
\end{array}\right]\right)^{-1}\right\|\left\|L_{0}^{-1}\right\| \leq \frac{1}{1-2 \sqrt{2}\left(1+k^{2}\right)^{2} k^{2} t}\left(2(1+k)^{2}\right) .
$$

In particular, for $t=t_{0},\left\|L_{t_{0}}^{-1}\right\| \leq 4(1+k)^{2}$. Since $C_{t}\left(\xi, \nabla_{t} \eta\right)=Q\left(L_{t} \xi, \nabla_{t} \eta\right)=$ $C_{t}\left(L_{t}^{-1} \nabla_{t}^{\prime} L_{t} \xi, \eta\right)$, the conjugate $\nabla_{t, C_{t}}^{\prime}$ of $\nabla_{t}$ with respect to $C_{t}$ is $\nabla_{t, C_{t}}^{\prime}=L_{t}^{-1} \nabla_{t}^{\prime} L_{t}$. We have

$$
\begin{gathered}
L_{t} \nabla_{t}=\left[\begin{array}{cc}
\left(I-R^{\prime} R\right) D_{1} & t\left(I-R^{\prime} R\right) R^{\prime}-(i \varepsilon+t S) R^{\prime} D_{2} \\
R(i \varepsilon+t S) D_{1} & t R(i \varepsilon+t S) R^{\prime}-D_{2}
\end{array}\right], \\
D_{1}^{\prime}\left(I-R^{\prime} R\right) \\
\nabla_{t}^{\prime} L_{t}=\left[\begin{array}{cc}
D_{1}^{\prime}(i \varepsilon+t S) R^{\prime} \\
-t R\left(I-R^{\prime} R\right)-D_{2}^{\prime} R(i \varepsilon+t S) & -t R(i \varepsilon+t S) R^{\prime}-D_{2}^{\prime}
\end{array}\right] .
\end{gathered}
$$

This implies that

$$
L_{t} \nabla_{t}+\nabla_{t}^{\prime} L_{t}=\left[\begin{array}{cc}
b & u_{t} \\
v_{t} & 0
\end{array}\right]
$$


where

$$
\begin{aligned}
b & =\left(I-R^{\prime} R\right) D_{1}-D_{1}\left(I-R^{\prime} R\right)=\left(D_{1} R^{\prime}-R^{\prime} D_{2}\right) R+R^{\prime}\left(D_{2} R-R D_{1}\right), \\
v_{t} & =R(i \varepsilon+t S) D_{1}-t R\left(I-R^{\prime} R\right)+D_{2} R(i \varepsilon+t S) \\
& =\left(D_{2} R-R D_{1}\right)(i \varepsilon+t S)+t R\left(D_{1} S+S D_{1}-\left(I-R^{\prime} R\right)\right)
\end{aligned}
$$

and

$$
\begin{aligned}
u_{t} & =-D_{1}(i \varepsilon+t S) R^{\prime}+t\left(I-R^{\prime} R\right) R^{\prime}-(i \varepsilon+t S) R^{\prime} D_{2} \\
& =-(i \varepsilon+t S)\left(R^{\prime} D_{2}-D_{1} R^{\prime}\right)-\left(D_{1} S+S D_{1}-\left(I-R^{\prime} R\right)\right) t R^{\prime}
\end{aligned}
$$

By assumptions $(a)$ and $(b), b, u_{t}$ and $v_{t}$ are in $\mathcal{L}_{G}(\mathcal{E})$. Thus

$$
\nabla_{t}+\nabla_{t, C_{t}}^{\prime}=L_{t}^{-1}\left(L_{t} \nabla_{t}+\nabla_{t}^{\prime} L_{t}\right) \in \mathcal{L}_{G}(\mathcal{E}), \quad t \in\left[0, t_{0}\right] .
$$

One can easily check that $\left(\mathcal{E}, C_{t}, \nabla_{t}\right)$ satisfies the other conditions. Hence $\left(\mathcal{E}, C_{t}, \nabla_{t}\right)$ $\in \mathcal{L}_{G, u}^{0}(\mathcal{A})$.

Step 3. We have shown that

$$
\left(\mathcal{E}, B_{t}, D\right) \in \mathcal{L}_{G, u}^{0}(\mathcal{A}[0,1]) \quad \text { and } \quad\left(\mathcal{E}, C_{t}, \nabla_{t}\right) \in \mathcal{L}_{G, u}^{0}\left(\mathcal{A}\left[0, t_{0}\right]\right) .
$$

Note that $\nabla_{0}=D$ and $C_{0}=B_{1},\left(\mathcal{E}, B_{1}, D\right)=\left(\mathcal{E}, C_{0}, \nabla_{0}\right)$. This implies that

$$
\psi_{0, u}((\mathcal{E}, Q, D))=\psi_{0, u}\left(\left(\mathcal{E}, B_{0}, D\right)\right)=\psi_{0, u}\left(\left(\mathcal{E}, B_{1}, D\right)\right)=\psi_{0, u}\left(\left(\mathcal{E}, C_{t_{0}}, \nabla_{t_{0}}\right)\right) .
$$

Consequently, it suffices to prove $\psi_{0, u}\left(\left(\mathcal{E}, C_{t_{0}}, \nabla_{t_{0}}\right)\right)=0$.

We now use Lemma 12(b).

(i) We have

$$
\begin{gathered}
\left\|\nabla_{t_{0}}^{2}\right\| \leq \tau_{1}^{2}: \nabla_{t}^{2}=\left[\begin{array}{cc}
D_{1}^{2} & t\left(D_{1} R^{\prime}-R^{\prime} D_{2}\right) \\
0 & D_{2}^{2}
\end{array}\right], \\
\left\|\nabla_{t_{0}}^{2}\right\| \leq\left\|D_{1}^{2}\right\|+t_{0}\left\|D_{1} R^{\prime}-R^{\prime} D_{2}\right\|+\left\|D_{2}^{2}\right\| \leq(2 \tau)^{2}, \tau_{1}=2 \tau .
\end{gathered}
$$

(ii) Since $2 k t_{0}=2 k \frac{1}{4 \sqrt{2}(1+k)^{2} k^{2}}<1$ and $12 \tau(1+k)^{3}<1$,

$$
\begin{aligned}
\left\|\nabla_{t_{0}}+\nabla_{t, C_{t_{0}}}^{\prime}\right\| & =\left\|L_{t_{0}}^{-1}\right\|\left\|L_{t_{0}} \nabla_{t_{0}}+\nabla_{t, C_{t_{0}}}^{\prime} L_{t_{0}}\right\| \leq\left\|L_{t_{0}}^{-1}\right\|\left(\|b\|+\left\|u_{t_{0}}\right\|+\left\|v_{t_{0}}\right\|\right) \\
& =4(1+k)^{2}\left(\left(\tau^{2} k+k \tau^{2}\right)+2 \tau^{2}\|i \varepsilon+t S\|+2 t_{0} k \tau^{2}\right) \\
& =\leq 8(1+k)^{2} \tau^{2}(3+k)<\tau_{1} .
\end{aligned}
$$

(iii) Take $X=\left[\begin{array}{cc}S & 0 \\ R / t_{0} & -Z\end{array}\right]$. Then

$$
\nabla_{t_{0}} X+X \nabla_{t_{0}}=\left[\begin{array}{cc}
D_{1} S+S D_{1}+R^{\prime} R & t_{0}\left(S R^{\prime}-R^{\prime} Z\right) \\
-\frac{1}{t_{0}}\left(D_{2} R-R D_{1}\right) & D_{2} Z+Z D_{2}+R R^{\prime}
\end{array}\right] .
$$

We obtain

$$
\begin{aligned}
&\left\|\nabla_{t_{0}} X+X \nabla_{t_{0}}-I\right\| \leq \sqrt{2} \max \left\{\left\|D_{1} S+S D_{1}+R^{\prime} R-I\right\|+\left\|t_{0}\left(S R^{\prime}-R^{\prime} Z\right)\right\|,\right. \\
&\left.\left\|\frac{1}{t_{0}}\left(D_{2} R-R D_{1}\right)\right\|+\left\|D_{2} Z+Z D_{2}+R R^{\prime}-I\right\|\right\} \\
& \leq \sqrt{2} \max \left\{\tau^{2}+2 t_{0} k^{2}, \frac{\tau^{2}}{t_{0}}+\tau^{2}\right\}<\frac{1}{2},
\end{aligned}
$$

since $\tau^{2}+\frac{1}{2 \sqrt{2}(1+k)^{2}}<\frac{1}{2 \sqrt{2}}$ and $\tau^{2}\left(4 \sqrt{2}(1+k)^{2} k^{2}+1\right)<\frac{1}{2 \sqrt{2}}$. Therefore, $\nabla_{t_{0}} X+$ $X \nabla_{t_{0}}$ is invertible and

$$
\left\|\left(\nabla_{t_{0}} X+X \nabla_{t_{0}}\right)^{-1}\right\| \leq \frac{1}{1-\left\|\nabla_{t_{0}} X+X \nabla_{t_{0}}-I\right\|} \leq 2 .
$$


(iv) The norm of $X$ can be estimated by

$$
\begin{aligned}
\|X\| & \leq \sqrt{2} \max \left\{\|S\|,\|Z\|+\left\|R / t_{0}\right\|\right\} \leq \sqrt{2}\left(k+\frac{k}{t_{0}}\right) \\
& =\sqrt{2}\left(k+4 \sqrt{2}(1+k)^{2} k^{3}\right) \stackrel{\text { def }}{=} k_{1} .
\end{aligned}
$$

(v) $\mathcal{E}$ already has the scalar product $\langle\xi, \eta\rangle=Q(\xi, T \eta)$ with $T^{2}=I$. Now $C_{t_{0}}(\xi, \eta)=Q\left(\xi, L_{t_{0}}^{\prime} \eta\right)=\left\langle\xi, T L_{t_{0}}^{\prime} \eta\right\rangle$. Clearly,

$$
\begin{aligned}
\left\|L_{t_{0}}^{\prime}\right\| & \leq \sqrt{2} \max \left\{\left\|I-R^{\prime} R\right\|+\left\|-i \varepsilon+t_{0} S^{\prime}\right\|\left\|R^{\prime}\right\|,\left\|R^{\prime}\left(-i \varepsilon+t_{0} S^{\prime}\right)\right\|+1\right\} \\
& \leq \sqrt{2}\left(1+k+k^{2}+\frac{1}{4 \sqrt{2}(1+k)^{2}}\right) .
\end{aligned}
$$

Also,

$$
\left\|\left(T L_{t_{0}}^{\prime}\right)\right\|\left\|\left(T L_{t_{0}}^{\prime}\right)^{-1}\right\| \leq 4 \sqrt{2}\left((1+k)^{3}+k^{2}(1+k)^{2}+\frac{1}{4 \sqrt{2}}\right) \leq k_{1}^{2} .
$$

(vi) Finally, we check that

$$
2 \sqrt{2} \tau_{1} k_{1}^{4}\left(k_{1}+3 k_{1}^{\frac{1}{2}}\right)<1,
$$

where $\tau_{1}=2 \tau, k_{1}=\sqrt{2}\left(k+4 \sqrt{2}(1+k)^{2} k^{3}\right)$. In fact,

$$
\begin{aligned}
2 \sqrt{2} \tau_{1} k_{1}^{4}\left(k_{1}+3 k_{1}^{\frac{1}{2}}\right) & =32 \tau\left(k+4 \sqrt{2}(1+k)^{2} k^{3}\right)^{5}\left(1+\frac{3}{\sqrt{\sqrt{2} k+8(1+k)^{2} k^{3}}}\right) \\
& \leq 64 \tau\left(k+4 \sqrt{2}(1+k)^{2} k^{3}\right)^{5}<1 .
\end{aligned}
$$

Hence, the condition of Lemma 12 is satisfied. The proof of the first part of the proposition is completed.

Step 4. We now prove the assertion with $(d)$ replaced by $\left(d^{\prime}\right)$. Let $Z_{1}=Z-$ $R R^{\prime} Z+R S W, X_{1}=I-R W-D_{2} Z-Z D_{2}, X_{2}=I-R^{\prime} R-D_{1} S-S D_{1}$. Then

$$
\left\|Z_{1}\right\| \leq\|Z\|+\|R\|\left\|R^{\prime}\right\|\|Z\|+\|R\|\|S\|\|W\| \leq k+2 k^{3},
$$

$$
\begin{aligned}
R S W & D_{2}+D_{2} R S W \\
& =\left(D_{2} R-R D_{1}\right) S W+R\left(D_{1} S+S D_{1}\right) W-R S\left(D_{1} W-W D_{2}\right) \\
& =\left(D_{2} R-R D_{1}\right) S W+R\left(I-R^{\prime} R\right) W-R X_{2} W-R S\left(D_{1} W-W D_{2}\right) .
\end{aligned}
$$

It follows that $\left\|R S W D_{2}+D_{2} R S W\right\| \leq \tau^{2} k^{2}+k\left(1+k^{2}\right) k+k \tau^{2} k+k^{2} \tau^{2}$. Also,

$$
\begin{aligned}
& R R^{\prime} Z D_{2}+D_{2} R R^{\prime} Z \\
& \quad=\left(D_{2} R-R D_{1}\right) R^{\prime} Z+R\left(D_{1} R^{\prime}-R^{\prime} D_{2}\right) Z+R R^{\prime}\left(D_{2} Z+Z D_{2}\right) .
\end{aligned}
$$

This yields $\left\|R R^{\prime} Z D_{2}+D_{2} R R^{\prime} Z\right\| \leq k^{2} \tau^{2}+k^{2} \tau^{2}+k^{2}\left(1+k^{2}+\tau^{2}\right)$. Now

$$
\begin{aligned}
R R^{\prime}- & I+Z_{1} D_{2}+D_{2} Z_{1} \\
= & R R^{\prime}-I+D_{2} Z+Z D_{2}+\left(R S W-R R^{\prime} Z\right) D_{2}+D_{2}\left(R S W-R R^{\prime} Z\right) \\
= & \left(R R^{\prime}-I\right) X_{1}-\left(D_{2} R-R D_{1}\right)\left(R^{\prime} Z-S W\right)-R\left(D_{1} R^{\prime}-R^{\prime} D_{2}\right) Z \\
& -R X_{2} W-R S\left(D_{1} W-W D_{2}\right) .
\end{aligned}
$$

We get

$$
\left\|R R^{\prime}-I+Z_{1} D_{2}+D_{2} Z_{1}\right\| \leq\left(1+k^{2}\right) \tau^{2}+\tau^{2}\left(2 k^{2}\right)+3 k^{2} \tau^{2}=\tau^{2}\left(1+6 k^{2}\right) .
$$


Therefore, replacing $\tau$ and $k$ in the first part of this proposition by $\tau \sqrt{1+6 k^{2}}$ and $k\left(1+2 k^{2}\right)$, we obtain that if

$$
64 \tau \sqrt{1+6 k^{2}}\left\{k\left(1+2 k^{2}\right)+4 \sqrt{2} k^{3}\left(1+2 k^{2}\right)^{3}\left(1+k\left(1+2 k^{2}\right)\right)^{2}\right\}^{5}<1,
$$

then the conclusion holds.

Let $M$ and $N$ be two closed, oriented Riemannian $G$-manifolds and $h: N \rightarrow M$ be a $G$-pseudo-equivalence. Let $E$ be a $\tau$-flat $G$ - $\mathcal{A}$-bundle over $M$ whose fiber is a finitely generated projective Hilbert $G$ - $\mathcal{A}$-module $E_{0}$, where $\tau>0$ is a constant. Let $\nabla$ be a $\tau$-flat connection on $E$. Then we have two elements $\left(\mathcal{E}_{E}, Q, D_{E}\right)$ and $\left(\mathcal{E}_{h^{*}(E)}, Q, D_{h^{*}(E)}\right)$ in $\mathcal{L}_{G, u}^{0}(\mathcal{A})$. Our goal in the rest of this section is to prove that for $\tau$ small, these elements satisfy the condition of Proposition 2. Hence, $G-\operatorname{Sig}\left(D_{E}\right)=G-\operatorname{Sig}\left(D_{h^{*}(E)}\right)$.

Lemma 13 ([HiS]). With the above notation, if $f: N \times[0,1] \rightarrow M$ is an equivariant smooth map, then there is a constant $k>0$ such that for every equivariant unitary connection $\nabla$ on $E,\left\|f_{0}^{*}(\nabla)-f_{1}^{*}(\nabla)\right\| \leq k\left\|\nabla^{2}\right\|$.

Proof. This lemma is independent of the group action, since we are concerned only with the norms. See the proof of Lemma 4.3 in [HiS].

The following lemma extends Lemmas 10 and 11 to the nonflat case.

Lemma 14 ([HiS]). Let $M$ and $N$ be two closed, oriented Riemannian $G$-manifolds and $E$ be a $G$-A-bundle over $M$ whose fiber is a finitely generated projective Hilbert $G$-A-module $E_{0}$.

(a) Let $h: N \rightarrow M$ be an equivariant smooth map and $\omega$ an equivariant smooth form on $N$. Denote by $N_{0}$ the support of $\omega$ and suppose that $h$ is a submersion near a $G$-neighborhood of $N_{0}$. Then $e_{\omega} h^{*}: \mathcal{E}_{E} \rightarrow \mathcal{E}_{h^{*}(E)}$ is a bounded equivariant operator. Moreover, the norm of $e_{\omega} h^{*}$ can be estimated by a number which is independent of $E$. Let $\nabla$ and $\nabla^{\prime}$ be equivariant unitary connections of $E$ and $h^{*}(E)$, respectively. Then $e_{\omega} h^{*}\left(\operatorname{dom}\left(D_{E}\right)\right) \subset \operatorname{dom}\left(\nabla^{\prime}\right)$ and $\nabla^{\prime}\left(e_{\omega} h^{*}\right)-\left(e_{\omega} h^{*}\right) \nabla \in$ $\mathcal{L}\left(\mathcal{E}_{E}, \mathcal{E}_{h^{*}(E)}\right)$.

(b) Let $h: N \times[0,1] \rightarrow M$ be an equivariant smooth map and $\omega$ an equivariant smooth form on $N$. Let $N_{0}$ be the support of $\omega$. Suppose that $h$ (resp., $h_{0}, h_{1}$ ) is a G-submersion near a neighborhood of $N_{0} \times[0,1]$ (resp., $N_{0}$ ). Then there is a constant $k>0$ such that for any connection $\nabla$ of $E$ there exists $R \in \mathcal{L}\left(\mathcal{E}_{E}, \mathcal{E}_{h_{0}^{*}(E)}\right)$ with $\|R\| \leq k$ and

$$
\left\|e_{\omega} h_{1}^{*}-e_{\omega} h_{0}^{*}-h_{0}^{*}(\nabla) R-R \nabla\right\| \leq k\left\|\nabla^{2}\right\| .
$$

Proof. (a) We refer to [HiS].

To prove part $(b)$, let

$$
R(\tilde{\xi})=\int_{0}^{1}\left(i_{\frac{\partial}{\partial t}}\left(e_{\omega} h^{*}(\tilde{\xi})\right)\right) d t, \tilde{\xi} \in \mathcal{E}_{E}
$$

Then the norm of $R$ can be estimated by the number $k$, which is independent of $h$ and the structures on $M$ and $N$. As the proof of Lemma 10,

$$
\left(h_{0}^{*}(\nabla) R+R \nabla\right)(\tilde{\xi})=\left(e_{\omega} h_{1}^{*}-e_{\omega} h_{0}^{*}\right)(\tilde{\xi})+\int_{0}^{1}\left(i_{\frac{\partial}{\partial t}}\left(e_{\omega}\left(h^{*}(\nabla)-h^{\prime *}(\nabla)\right)(\tilde{\xi})\right)\right) d t,
$$


where $h^{\prime}(x, t)=h(x, 0): N \times[0,1] \rightarrow M$. Let $R_{1}$ be defined by the second term of the above identity. Then

$$
e_{\omega} h_{1}^{*}-e_{\omega} h_{0}^{*}=h_{0}^{*}(\nabla) R+R \nabla+R_{1} .
$$

To estimate the norm of $R_{1}$, let $H(x, t, \lambda)=h(x, \lambda t)$. Then $H(x, t, 0)=h^{\prime}(x, t)$ and $H(x, t, 1)=h(x, t)$. Using Lemma 13 , we get $\left\|R_{1}\right\| \leq k\left\|\nabla^{2}\right\|$.

Let $P: N \times B^{k} \rightarrow M$ be a $G$-submersion, $\tau$ a $G$-smooth form of mass 1 on $B^{k}$. Suppose that $q: N \times B^{k} \rightarrow N$ and $r: N \times B^{k} \rightarrow B^{k}$ are the projections, $\omega=r^{*}(\tau)$, and $h: N \rightarrow M$ is the restriction to $N \times\{o\}$ of $P$. Then $h q: N \times B^{k} \rightarrow M$ is equivariantly homotopic to $P$ via the homotopy $H(x, t, \lambda)=P(x, \lambda t)$. For this reason, we identify $P^{*}(E)$ with $q^{*} h^{*}(E)$. Let $R_{P, \tau}=R_{P, \tau}(E, \nabla)=q ! e_{\omega} P^{*} \in$ $\mathcal{L}_{G}\left(\mathcal{E}_{E}, \mathcal{E}_{h^{*}(E)}\right)$.

Lemma 15 ([HiS]). (a) Let $P^{\prime}: N \times B^{l} \rightarrow M$ be another $G$-submersion and $\tau^{\prime}$ a $G$-smooth form of mass 1 on $B^{l}$. Suppose $h(x)=P(x, o)$ and $h^{\prime}(x)=P^{\prime}(x, o)$ are equivariantly homotopic. Then there exists a constant $k>0$ such that for each pair $(E, \nabla)$ as above there is $R \in \mathcal{L}_{G}\left(\mathcal{E}_{E}, \mathcal{E}_{h^{*}(E)}\right)$ satisfying $\|R\| \leq k$ and

$$
\left\|R \nabla+\nabla R+R_{P, \tau}(E, \nabla)-R_{P^{\prime}, \tau^{\prime}}(E, \nabla)\right\| \leq k\left\|\nabla^{2}\right\| .
$$

(b) Suppose $\tilde{P}^{\prime}: O \times B^{l} \rightarrow N$ is a G-submersion and $\tau^{\prime}$ a $G$-smooth form of mass 1 on $B^{l}$. Let $P^{\prime \prime}: O \times B^{l} \times B^{k} \rightarrow M$ be defined by $P^{\prime \prime}(x, s, t)=P\left(\tilde{P}^{\prime}(x, s), t\right)$ and $\tau^{\prime \prime}=\tau^{\prime} \times \tau$. Then there exists a constant $k>0$ such that for each pair $(E, \nabla)$,

$$
\left\|R_{P^{\prime}, \tau^{\prime}}\left(h^{*}(E), h^{*}(\nabla)\right) R_{P, \tau}(E, \nabla)-R_{P^{\prime \prime}, \tau^{\prime \prime}}(E, \nabla)\right\| \leq k\left\|\nabla^{2}\right\| .
$$

Proof. (a) By Lemma 14, the proof is the same as that of Lemma 11.

(b) Define $f: O \rightarrow M$ by $f(x)=P^{\prime \prime}(x, o, o)$ and let $q^{\prime \prime}: O \times B^{k} \times B^{l} \rightarrow O$ be the projection. Define two homotopies $H$ and $\bar{H}$ between $P^{\prime \prime}$ and $f q^{\prime \prime}$ from $O \times B^{l} \times B^{k} \times[0,1]$ to $M$ by

$$
H(x, s, t, \lambda)=P^{\prime \prime}(x, \lambda s, \lambda t)
$$

and

$$
\bar{H}(x, s, t, \lambda)=P^{\prime \prime}(x, \sup (0,2 \lambda-1) s, \inf (1,2 \lambda) t) .
$$

We have $H(x, s, t, o)=\left(f q^{\prime \prime}\right)(x, s, t), H(x, s, t, 1)=P^{\prime \prime}(x, s, t)$. We can pull back the metric-preserving connection $\nabla$ on $E$ to a metric-preserving connection $H(\nabla)$ on $H^{*}(E)$ via the smooth map $H$, and then by the parallel transport along $[0,1]$ we define a unitary operator $U \in \mathcal{L}_{G}\left(\mathcal{E}_{P^{\prime \prime *}(E)}, \mathcal{E}_{\left(f q^{\prime \prime}\right)^{*}(E)}\right)$. The same reasoning shows that $\bar{H}$ produces unitary operators in $\mathcal{L}_{G}\left(\mathcal{E}_{P^{\prime \prime *}(E)}, \mathcal{E}_{H_{\frac{1}{2}}^{*}(E)}\right)$ and $\mathcal{L}_{G}\left(\mathcal{E}_{H_{\frac{1}{2}}^{*}(E)}, \mathcal{E}_{\left(f q^{\prime \prime}\right)^{*}(E)}\right)$, hence a unitary operator $\bar{U} \in \mathcal{L}_{G}\left(\mathcal{E}_{P^{\prime \prime *}(E)}, \mathcal{E}_{\left(f q^{\prime \prime}\right)^{*}(E)}\right)$, where $H_{\frac{1}{2}}^{\frac{1}{2}}=H\left(x, s, t, \frac{1}{2}\right)$. By definition, $R_{P^{\prime \prime}, \tau^{\prime \prime}}=q_{!}^{\prime \prime} U e_{\omega^{\prime \prime}}\left(P^{\prime \prime}\right)^{*}$ for $\omega^{\prime \prime}=\pi_{l, k}^{*}\left(\tau^{\prime \prime}\right)$, where $\pi_{l, k}: O \times B^{l} \times B^{k} \rightarrow$ $B^{l} \times B^{k}$ is the projection. Let $\bar{R}_{P^{\prime \prime}, \tau^{\prime \prime}}=q_{!}^{\prime \prime} \bar{U} e_{\omega^{\prime \prime}}\left(P^{\prime \prime}\right)^{*}$. Then, as in the proof of Lemma $11(b), \bar{R}_{P^{\prime \prime}, \tau^{\prime \prime}}=R_{P^{\prime}, \tau^{\prime}} R_{P, \tau}$. The result follows easily from [HiS]:

$$
\left\|\bar{R}_{P^{\prime \prime}, \tau^{\prime \prime}}-R_{P^{\prime \prime}, \tau^{\prime \prime}}\right\| \leq\left\|q_{!}^{\prime \prime}(\bar{U}-U) e_{\omega^{\prime \prime}}\left(P^{\prime \prime}\right)^{*}\right\| \leq K_{1}\|\bar{U}-U\| \leq k_{1} m\left\|\nabla^{2}\right\|,
$$

where $k_{1}$ and $m$ are constants independent of $\nabla$ and $E$.

Finally, we prove the equivariant Connes-Gromov-Moscovici theorem. 
Proof of Theorem 2. We use Proposition 2 for $\left(\mathcal{E}_{E}, Q, D_{E}\right)$ and $\left(\mathcal{E}_{h^{*}(E)}, Q, D_{h^{*}(E)}\right)$. Let $J: M \rightarrow \mathbb{R}^{k}$ be a $G$-embedding, $O$ a $G$-tubular neighborhood of $J(M)$ in $\mathbb{R}^{k}$ and $\pi: O \rightarrow M$ the corresponding $G$-projection. Suppose $J(M)+B^{k} \subset O$. Let $P: N \times B^{k} \rightarrow M$ be the $G$-submersion given by $P(x, t)=\pi(h(x)+t)$, and $\tau$ a $G$-volume form of mass 1 on $B^{k}$. Take $R=R_{P, \tau} \in \mathcal{L}_{G}\left(\mathcal{E}_{E}, \mathcal{E}_{h^{*}(E)}\right)$. We now verify the condition of Proposition 2 . To check condition $(a)$, let $\tau_{1}^{2} \geq\left\|\nabla^{2}\right\|$. Then $\left\|D_{E}^{2}\right\|=\left\|\nabla^{2}\right\| \leq \tau_{1}^{2}$, and $\left\|D_{h^{*}(E)}\right\|=\left\|\nabla^{2}\right\| \leq \tau_{1}^{2}$. Take a $G$-smooth function $\varphi$ with compact support such that $\varphi \omega=\omega$ on $N \times B^{k}$. By Lemma 14, we can estimate $\left\|e_{\omega} P^{*}\right\|$ and $\left\|q_{!, \varphi}\right\|=\left\|\left(e_{\omega} P^{*}\right)^{\prime}\right\|=\left\|e_{\omega} P^{*}\right\|$. Clearly,

$$
P^{*} \nabla=P^{*}(\nabla) P^{*}, \quad q^{*} h^{*}(\nabla)=(h q)^{*}(\nabla) q^{*}
$$

and $e_{\omega} P^{*}(\nabla)=P^{*}(\nabla) e_{\omega}$ for the closed form $\omega$. Then

$$
\left(e_{\varphi} q^{*}\right)^{\prime} e_{\omega} P^{*} \nabla=\left(e_{\varphi} q^{*}\right)^{\prime} P^{*}(\nabla) e_{\omega} P^{*},
$$

and

$$
\begin{aligned}
&\left\|\left(e_{\varphi} q^{*}\right)^{\prime} e_{\omega} P^{*} \nabla-h^{*}(\nabla)\left(e_{\varphi} q^{*}\right)^{\prime} e_{\omega} P^{*}\right\| \\
&=\left\|\left(e_{\varphi} q^{*}\right)^{\prime} P^{*}(\nabla) e_{\omega} P^{*}-h^{*}(\nabla)\left(e_{\varphi} q^{*}\right)^{\prime} e_{\omega} P^{*}\right\| \\
& \leq\left\|\left(e_{\varphi} q^{*}\right)^{\prime}\left(P^{*}(\nabla)-(h q)^{*}(\nabla)\right) e_{\omega} P^{*}\right\| \\
&+\left\|\left(h^{*}(\nabla)\left(e_{\varphi} q^{*}\right)^{\prime}-\left(e_{\omega} q^{*}\right)^{\prime}(h q)^{*}(\nabla)\right) e_{\omega} P^{*}\right\| \\
& \leq m\left\|\nabla^{2}\right\|,
\end{aligned}
$$

by Lemmas 13 and 14 and the fact that $h^{*}(\nabla)\left(e_{\varphi} q^{*}\right)^{\prime}=\left(e_{\varphi} q^{*}\right)^{\prime}(h q)^{*}(\nabla)$. We have $R\left(\operatorname{dom}\left(D_{E}\right)\right) \subset \operatorname{dom}\left(D_{h^{*}(E)}\right)$ and $\left\|R D_{E}-D_{h^{*}(E)} R\right\| \leq m_{1}\left\|\nabla^{2}\right\|$ for some $m_{1}>0$ independent of $(E, \nabla)$.

To check condition $(b)$, let $q_{i}: N \times B^{k} \times B^{k} \rightarrow N \times B^{k}$ be given by $q_{i}\left(x, t_{1}, t_{2}\right)=$ $\left(x, t_{i}\right), P_{1}: M \times B^{k} \rightarrow M$ be the projection and $\tilde{\pi}: M \times B^{k} \rightarrow M$ be the $G$ submersion given by $\tilde{\pi}(x, t)=\pi(x+t)$. Then we can argue as the proof of Theorem 1 by using Proposition 2 to get $S \in \mathcal{L}_{G}(\mathcal{E})$ such that $\|S\| \leq m_{2}, S\left(\operatorname{dom}\left(D_{E}\right)\right) \subset$ $\operatorname{dom}\left(D_{E}\right)$ and

$$
\left\|I-R^{\prime} R-D_{E} S-S D_{E}\right\| \leq m_{3}\left\|\nabla^{2}\right\|
$$

for constants $m_{i}>0$ independent of $(E, \nabla)$. The rest of the proof is the same as that of Theorem 1.

To check condition $(c)$, take $\varepsilon(\tilde{\xi})=(-1)^{\partial \tilde{\xi}} \tilde{\xi}$. Then clearly $\varepsilon\left(\operatorname{dom}\left(D_{E}\right)\right) \subset$ $\operatorname{dom}\left(D_{E}\right), \varepsilon D_{E}=-D_{E} \varepsilon, \varepsilon=\varepsilon^{\prime}, \varepsilon^{2}=I$ and $\varepsilon\left(I-R^{\prime} R\right)=\left(I-R^{\prime} R\right) \varepsilon$. See the corresponding part of Theorem 1 .

Finally we verify condition $\left(d^{\prime}\right)$ of Proposition 2 . Indeed, let $P_{1}: M \times B^{l} \rightarrow N$ be a submersion such that $q_{1}: M \rightarrow N$ given by $q_{1}(x)=P_{1}(x, o)$ is a homotopic inverse of $h$, i.e., $q_{1} h$ and $h q_{1}$ are homotopic to the identities, respectively. Note that $P_{1}$ and $q_{1}$ may not be equivariant, and homotopy between $q_{1} h$ and the identity may not be equivariant either. However, we can still identify $E$ with $\left(h q_{1}\right)^{*}(E)$ non-equivariantly. Let $\tau^{\prime}$ be a volume form of mass 1 on $B^{l}$ and $W_{1}=R_{P_{1}, \tau^{\prime}} \in$ $\mathcal{L}_{G}\left(\mathcal{E}_{h^{*}(E)}, \mathcal{E}_{E}\right)$. Then, as before, there are constants $m_{4}, m_{5}>0$ such that $\left\|W_{1}\right\| \leq$ $m_{5}, W_{1}\left(\operatorname{dom}\left(D_{E}\right)\right) \subset \operatorname{dom}\left(D_{E}\right)$ and

$$
\left\|W_{1} D_{h^{*}(E)}-D_{E} W_{1}\right\| \leq m_{4}\left\|\nabla^{2}\right\| .
$$


By Lemma 15 and Step 3 of the proof of Theorem 1, there exists $Z_{1} \in \mathcal{L}_{G}\left(\mathcal{E}_{h^{*}(E)}\right)$ satisfying $\left\|Z_{1}\right\| \leq m_{6}, Z_{1}\left(\operatorname{dom}\left(D_{h^{*}(E)}\right)\right) \subset \operatorname{dom}\left(D_{h^{*}(E)}\right)$ and

$$
\left\|I-R W_{1}-D_{h^{*}(E)} Z_{1}-Z_{1} D_{h^{*}(E)}\right\| \leq m_{7}\left\|\nabla^{2}\right\|,
$$

where $m_{6}, m_{7}$ are positive constants independent of $(E, \nabla)$. Let $W=\int_{G} g\left(W_{1}\right) d g$, $Z=\int_{G} g\left(Z_{1}\right) d g$. We get the required operators. Let

$$
\tau^{2}=\max \left\{\left\|\nabla^{2}\right\|, m_{1}\left\|\nabla^{2}\right\|, m_{3}\left\|\nabla^{2}\right\|, m_{4}\left\|\nabla^{2}\right\|, m_{7}\left\|\nabla^{2}\right\|\right\}
$$

and $k=\max \left\{m_{0}, m_{2}, m_{5}, m_{6}\right\}$. Then we have verified the condition of Proposition 2 as long as $\left\|\nabla^{2}\right\|$ is sufficiently small.

We refer to Section 3 for the main idea of the above proof. We should point out that the argument in Section 3 and this section closely follows [HiS], except that our estimates are slightly different. We called this the equivariant Hilsum-Skandalis method in the Introduction.

The following is an example of $\tau$-flat $G$-vector bundles (see also [Ska]).

Example 1. For simplicity, we take $G=S^{1}$ and $M_{1}=S^{1}$ with a trivial $G$-action. Let $E_{1}=S^{1} \times \mathbb{R}^{m}$ be a trivial vector bundle over $M_{1}$ with the $G$-action on a fiber given by the multiplication $e^{2 \pi i t} \in S^{1}$. Choose a basis $\left\{e_{1}, \cdots, e_{m}\right\}$ for $\mathbb{R}^{m}$. Let $l>0$ be an integer. Define a parallel transport $\sigma_{[r, s]}$ along the oriented segment $[r, s] \subset \mathbb{R}^{1}$ by the following formula:

$$
\sigma_{[r, s]}\left(e_{j}\right)=e^{\frac{2 \pi i(s-r)(j-1) l}{m}} e_{j}, \quad j=1,2, \cdots, m .
$$

It is easy to check that the connection $\nabla$ associated with this parallel transport is $G$-invariant and flat. Let $u: S^{1} \rightarrow U\left(E_{1}\right)$ be the section of the bundle $U\left(E_{1}\right)$ of unitary endomorphisms on $E_{1}$ given by $u(t) e_{i}=e_{i-1}, i \neq 1$, and $u(t) e_{1}=e^{2 \pi i t l} e_{m}$. Clearly, $u$ is $G$-equivariant. Hence it determines an element $[u] \in K_{G}^{1}\left(M_{1}\right)$. It is easy to check that

$$
\sigma_{\left[t_{0}, t\right]} u\left(t_{0}\right) \sigma_{\left[t, t_{0}\right]}=e^{-\frac{2 \pi i\left(t-t_{0}\right) l}{m}} u(t) .
$$

Let us estimate $\|\nabla(u)\|$ :

$$
\begin{aligned}
\|\nabla(u)\| & =\max _{x_{0} \in M_{1}, X \in T_{x_{0}} M_{1},\|X\| \leq 1}\left\|\nabla_{X}(u)\right\|_{\left(E_{1}\right)_{x_{0}}} \\
& =\max _{\substack{x_{0} \in M_{1}, X \in T_{x_{0}} M_{1},\|X\| \leq 1 \\
p_{0} \in\left(E_{1}\right)_{x_{0}},\left\|p_{0}\right\| \leq 1}}\left\|\lim _{t \rightarrow t_{0}} \frac{\sigma_{\left[t, t_{0}\right]} u(t) \sigma_{\left[t_{0}, t\right]}\left(p_{0}\right)-u\left(t_{0}\right)\left(p_{0}\right)}{t-t_{0}}\right\|_{\left(E_{1}\right)_{x_{0}}} \\
& =\max \left\|\lim _{t \rightarrow t_{0}} \frac{e^{2 \pi i\left(t-t_{0}\right) l / m}-1}{t-t_{0}} u\left(t_{0}\right)\left(p_{0}\right)\right\|_{\left(E_{1}\right)_{x_{0}}} \\
& \leq 2 \pi l / m .
\end{aligned}
$$

We may use $[u] \in K_{G}^{1}\left(M_{1}\right)$ to produce an element in $K_{G}^{0}\left(S^{2}\right)$. Indeed, let $S^{2}$ be identified with $\mathbb{C} \cup \infty$. Let $E_{2}$ and $E_{3}$ be the trivial vector bundles over the interior and exterior of the unit circle $S^{1}$ with the fiber $\mathbb{R}^{m}$. Using $u: S^{1} \rightarrow U\left(\mathbb{R}^{m}\right)$ as a gluing map, we may glue the bundles $E_{2}$ and $E_{3}$ together along $S^{1}$ and get a new bundle $E$ over $S^{2}$. This procedure also yields a connection on $E$ which is $\tau$-flat for $m \rightarrow \infty$, because $\|\nabla(u)\| \leq 2 \pi l / m$.

In general, one may construct $\tau$-flat $G$-bundles from those in the non-equivariant cases as long as the constructions in the latter cases can be made equivariantly. 


\section{Equivariant Novikov Conjecture for Euclidean Buildings}

We begin with a definition of the equivariant Novikov conjecture. Let $M$ be a closed, oriented Riemannian manifold and $G$ a compact Lie group acting on $M$ by isometries. Denote by $\pi(M)$ the fundamental groupoid of $M$, which is defined to be the set of homotopy classes (rel endpoints) of all paths in $M . \pi(M)$ can be given by $\pi(M)=\frac{\tilde{M} \times \tilde{M}}{\pi_{1}(M)}$, where $\tilde{M}$ is the universal covering space of $M$. $G$ acts naturally on $\pi(M)$. Let $B \pi(M)$ be the equivariant classifying space of $\pi(M)$ (cf. May's appendix to [RoW2]) and $f_{M}: M \rightarrow B \pi(M)$ be the equivariant classifying map. If $h: N \rightarrow M$ is a $G$-pseudo-equivalence from another closed, oriented Riemannian $G$-manifold $N$, i.e., $h$ is $G$-invariant and is a homotopy equivalence, then $h$ induces the maps $h_{*}: \pi(N) \rightarrow \pi(M)$ and $h_{*}: B \pi(N) \rightarrow B \pi(M)$. Hence the following diagram of equivariant $K$-homology groups is commutative:

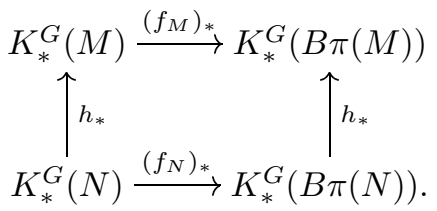

Let $D_{M}$ be the equivariant signature operator on $M$. Then $D_{M}$ defines an element $\left[D_{M}\right]$ in $K_{*}^{G}(M)$, which is in $K_{0}^{G}(M)$ for the even dimensional $M$ and in $K_{1}^{G}(M)$ otherwise. Although the identity $h_{*}\left(f_{N}\right)_{*}\left(\left[D_{N}\right]\right)=\left(f_{M}\right)_{*}\left(\left[D_{M}\right]\right)$ is not true in general, in view of $(7)$, one may still expect that this identity holds for some special cases. More generally, given a $G$-equivariant commutative diagram

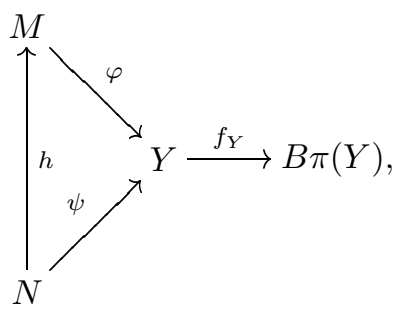

where $Y$ is a $G$-space, $h$ is a $G$-pseudo-equivalence, and $\varphi$ and $\psi$ are $G$-equivariant maps, one conjectures the following:

$\underline{E N C_{Y}}([\mathrm{RoW} 2]) \cdot\left(f_{Y}\right)_{*} \varphi_{*}\left(\left[D_{M}\right]\right)=\left(f_{Y}\right)_{*} \psi_{*}\left(\left[D_{N}\right]\right)$ in $K_{*}^{G}(B \pi(Y))$, provided $\left.\overline{K_{*}^{G}(B \pi}(Y)\right)$ is finitely generated over the representation $\operatorname{ring} R(G)$ of $G$.

Rosenberg and Weinberger have proved this conjecture for $Y$ a complete Riemannian manifold of nonpositive curvature, provided $K_{*}^{G}(Y)$ is a finitely generated module over $R(G)$. The condition on $K_{*}^{G}(Y)$ plays an important role in [RoW2], due to the lack of the equivariant Mishchenko symmetric signature for general compact Lie group actions and other technical difficulties. The unsolved problem in this case is to remove the condition on $K_{*}^{G}(Y)$ and to prove the existence of the equivariant maps $\varphi$ and $\psi$ in (8) for a general manifold $M$. On the other hand, it is desirable to verify $E N C_{Y}$ for those $Y$ whose universal coverings are equivariantly isomorphic to the geometric realizations of Euclidean buildings, since Euclidean buildings are natural analogues of complete Riemannian manifolds of nonpositive curvature. The recent work of Kasparov-Skandalis [KaS] on Euclidean buildings enables us to verify $E N C_{Y}$ for such $Y$. The goal of this section is to carry out the 
proof of the equivariant Novikov conjecture for the above mentioned $Y$ by using Theorem 1 in Section 3. Meanwhile, we will also get rid of the assumption on $K_{*}^{G}(Y)$. The existence of the $G$-maps $\varphi$ and $\psi$ in (8) will be considered in Section 6.

We now recall the definition of Euclidean buildings (cf. [Bro] [KaS] [Tits] for more details). Let $X$ be a simplicial complex of dimension $n$ and $B$ its geometric realization. $X$ can be considered as a set of its faces, $X=\bigcup_{0 \leq k \leq n} X^{k}$. $X$ is said to be typed if there is a map $\theta: X^{0} \rightarrow\{0,1, \ldots, n\}$ such that for every simplex $x \in X$, the images under $\theta$ of the vertices of $x$ are pairwise different. $\theta$ is called a type of $X$. There is a typed simplicial complex $X_{1}$ associated with a given simplicial complex $X$ of dimension $n$ such that $X_{1}$ and $X$ have the same geometric realization. We use the notation that chambers are the simplices of dimension $n$; walls are the simplices of dimension $n-1$, and apartments are subcomplexes of $X$ determined by the Weyl system.

Definition 4. $(X, B)$ is called a Euclidean building if

(i) $B$ has a metric such that the apartments are affinely isometric to the Euclidean space $\mathbb{R}^{n}$;

(ii) any pair of simplices of $X$ is contained in an apartment;

(iii) the intersection $S \cap S^{\prime}$ of any two apartments $S$ and $S^{\prime}$ is convex and there is a simplicial isometry $j: S \rightarrow S^{\prime}$ such that $j$ is the identity map on $S \cap S^{\prime}$ and preserves the type, i.e., $\theta(j(x))=\theta(x), \forall x \in S$;

(iv) for any two chambers $\sigma$ and $\sigma^{\prime}$ of an apartment $S$ there is a type-preserving simplicial isometry $j: S \rightarrow S$ mapping $\sigma$ to $\sigma^{\prime}$.

This definition of a Euclidean building is slightly different from the one in [Tits]. In fact, every Bruhat-Tits Euclidean building is a Euclidean building in the above sense.

Example 2. (a) Let $F$ be a field with a discrete valuation and $S L_{n}(F)$ the group of $n \times n$ matrices over $F$ with determinant 1 . Then we can associate with $S L_{n}(F)$ a Euclidean building. In particular, this building for $S L_{2}(\mathbb{Q})$ is a tree [Bro].

(b) The universal covering space of a complete Riemannian manifold of nonpositive curvature can be considered as a geometric realization of some topological building $[\mathrm{BuS}]$. For more examples of Euclidean buildings we refer to [Bro].

Note that the geometric realization $B$ of a Euclidean building $X$ is contractible [Bro]. Let $\Gamma_{0}$ be a discrete group which acts properly and freely on the building $(X, B)$ by type-permuting isometries. Here the action of $\Gamma_{0}$ is called typepermuting if there is a group homomorphism $\tilde{\pi}: \Gamma_{0} \rightarrow S_{n}$, the permutation group of $\{0,1, \ldots, n\}$, such that $\theta(g(x))=\tilde{\pi}(g) \theta(x), \forall g \in \Gamma_{0}, x \in X$. The action is called type-preserving if $\tilde{\pi}(g)=I, \forall g \in \Gamma_{0}$. Then the universal covering space of $Y=B / \Gamma_{0}$ is $B$. We assume that $G$ acts on $Y$ by isometries via a homomorphism of $G$ into $I \operatorname{som}(Y)$, the isometry group of $Y$, such that the lifting of the $G$-action to $B$ is type-permuting. More precisely, there is a locally compact group $\Gamma$ in $\operatorname{Isom}(B)$ such that $\Gamma$ is a group extension of $\Gamma_{0}$ by $G, 1 \rightarrow \Gamma_{0} \rightarrow \Gamma \rightarrow G \rightarrow 1$, and $\Gamma$ acts on $B$ by type-permuting isometries. The importance of $\Gamma$ is that $C_{r}^{*}(\Gamma)$ is strongly Morita equivalent to the semidirect product $C_{r}^{*}(\pi(Y)) \rtimes G$ of $C_{r}^{*}(\pi(Y))$ and $G$ [RoW2], where $C_{r}^{*}(\Gamma)$ (resp., $C_{r}^{*}(\pi(Y))$ ) is the reduced group (resp., groupoid) $C^{*}$-algebra 
of $\Gamma$ (resp., $\pi(Y))$. Hence

$$
K_{*}^{G}\left(C_{r}^{*}(\pi(Y))\right) \simeq K_{*}\left(C_{r}^{*}(\pi(Y)) \rtimes G\right) \simeq K_{*}\left(C_{r}^{*}(\Gamma)\right) .
$$

To consider the $E N C_{Y}$ for $Y=B / \Gamma_{0}$, we need a non-Hausdorff smooth manifold $M_{X}$ associated with $(X, B)$. Let us first give the following general definition.

Definition 5. We call $\left(U_{i}, U_{i j}, \varphi_{i j}\right)_{i, j \in J}$ a $\Gamma$-atlas if it satisfies the following:

(i) $\Gamma$ acts on the index set $J$ and $U_{i}$ such that $g\left(U_{i}\right)=U_{g(i)}, i \in J, g \in \Gamma$;

(ii) each $U_{i}$ is a Hausdorff smooth manifold;

(iii) $U_{i i}=U_{i}$, and for $i, j \in J, U_{i j}$ is an open subset in $U_{i}$;

(iv) $\varphi_{i j}: U_{j i} \rightarrow U_{i j}$ is a diffeomorphism such that $\varphi_{i i}=I_{U_{i}}$ and $\varphi_{i j}(g x)=$ $g \varphi_{i j}(x), g \in \Gamma$.

We may associate a manifold $M_{0}$ to a $\Gamma$-atlas $\left(U_{i}, U_{i j}, \varphi_{i j}\right)_{i, j \in J}$ by taking $M_{0}$ to be the quotient of the disjoint union of $U_{i}, i \in J$, by the equivalent relation " $x \sim$ $\varphi_{i j}(x)$ ", $x \in U_{j i} . M_{0}$ is in general non-Hausdorff. As one can easily check, $M_{0}$ is Hausdorff iff the maps $(r, s): U_{i j} \rightarrow U_{i} \times U_{j}$ given by $r(x)=x$ and $s(x)=r\left(\varphi_{j i}(x)\right)$ for $x \in U_{i j}$ are proper.

The non-Hausdorff manifold $M_{X}$ associated to the building $(X, B)$ can be defined as follows. Let $E$ be the affine Euclidean space $E=\left\{t=\left(t_{0}, \ldots, t_{n}\right) \in \mathbb{R}^{n+1}\right.$ : $\left.\sum_{i=0}^{n} t_{i}=1\right\}$ and $\Sigma=\left\{t \in E: t=\left(t_{0}, \ldots, t_{n}\right), t_{i} \geq 0, \forall i\right\}$. Since $\Sigma$ is a convex set in $E$, we can define a continuous map $q: E \rightarrow E$ by the formula $\|q(t)-t\|=$ $\inf \{\|t-s\|: s \in \Sigma\}$. For a subset $O$ of $\{0,1, \ldots, n\}$ let $F_{O}$ be the face of $\Sigma$ defined by $F_{O}=\left\{t \in \Sigma: t=\left(t_{0}, \ldots, t_{n}\right): i \in O\right.$ if $\left.t_{i} \neq 0\right\}$. Denote by $\Omega_{O}$ the interior in $E$ of $q^{-1}\left(F_{O}\right)$. Obviously, $F_{\emptyset}=\Omega_{\emptyset}=\emptyset$. Let $U_{x}=E, x \in X, U_{x, y}=\Omega_{\theta(x \cap y)}$ for $x \neq y$ in $X$ and $\varphi_{x, y}=I$. Then $\left\{U_{x}, U_{x, y}, \varphi_{x, y}\right\}_{x, y \in X}$ is a $\Gamma$-atlas. We define $M_{X}=U^{0} / \sim$, where $U^{0}$ stands for the disjoint union of the $U_{x}, x \in X$. $M_{X}$ is a non-Hausdorff smooth $\Gamma$-manifold endowed with a $\Gamma$-invariant Riemannian metric, since its tangent bundle is trivial. Indeed, fibers of the tangent bundle of $M_{X}$ are the space of tangent vectors to $E$. The crucial property of $M_{X}$ is that $M_{X}$ is $\Gamma$ equivariantly homotopy equivalent to $B$ (cf. [KaS], Lemma 4.8), i.e., there are $\Gamma$ equivariant maps $f_{1}: M_{X} \rightarrow B$ and $f_{2}: B \rightarrow M_{X}$ such that $f_{1} f_{2}$ and $f_{2} f_{1}$ are $\Gamma$ equivariantly homotopic to the identity maps, respectively. We have the diagram

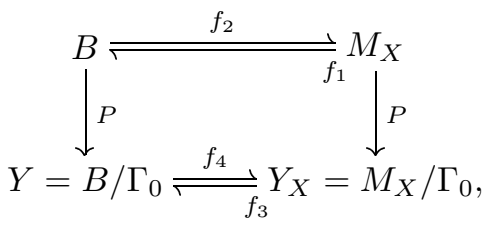

where $P$ is the natural projection; $f_{3}$ and $f_{4}$ are defined by the commutativity of the diagram. Then $Y$ is $G$-equivariantly homotopy equivalent to $Y_{X}$ via the maps $f_{3}$ and $f_{4}$. The advantage of introducing $Y_{X}$ is that one can use various $K K$-theoretic information about non-Hausdorff special manifolds. We now reduce $E N C_{Y}$ to the following problems:

(1) to construct an injective map $\beta: K_{*}^{G}(Y) \rightarrow K_{*}^{G}\left(C_{r}^{*}(\pi(Y))\right)$;

(2) to show the $G$-pseudo-equivalence of $\beta\left(\varphi_{*}\left(\left[D_{M}\right]\right)\right)$.

To define the map $\beta$, let us assume that $G$ has a fixed point in $Y$. This condition is not necessary for the construction of $\beta$, but it will be needed in Proposition 3 below. So we use it here as well to simplify the discussion. Thus $G$ acts on the 
fundamental group $\Gamma_{0}$ of $Y$, and $\Gamma=\Gamma_{0} \rtimes G$, a semidirect product of $\Gamma_{0}$ and $G$. By Theorem 2.5 of [RoW2], $K_{*}^{G}\left(C_{r}^{*}(\pi(Y))\right)=K_{*}^{G}\left(C_{r}^{*}\left(\Gamma_{0}\right)\right)$. Let $V$ be the $G$ $C_{r}^{*}\left(\Gamma_{0}\right)$-bundle over $Y$ whose fiber $V_{y}$ at $y \in Y$ is the $C^{*}$-algebra $C_{r}^{*}\left(\Gamma_{0}\right)$, namely, $V=B \times_{\Gamma_{0}} C_{r}^{*}\left(\Gamma_{0}\right) . V$ is a flat $G-C_{r}^{*}\left(\Gamma_{0}\right)$-bundle. Note that the restriction of $V$ to a $G$-compact subset $Z \subset Y$ yields a $G$ - $C_{r}^{*}\left(\Gamma_{0}\right)$-bundle over $Z$, and hence defines an element $\left[\beta_{Z}\right]$ in $K_{0}^{G}\left(C^{*}(Z) \otimes C_{r}^{*}\left(\Gamma_{0}\right)\right)$. Let $\beta_{Z}^{*}$ be the homomorphism from $K_{*}^{G}(Z)$ to $K_{*}^{G}\left(C_{r}^{*}\left(\Gamma_{0}\right)\right)$ given by the Kasparov product with $\left[\beta_{Z}\right]$. The map $\beta_{Z}^{*}$ respects the inductive limit and gives rise to a map

$$
\beta: K_{*}^{G}(Y)=\lim _{\rightarrow Z \subset Y} K_{*}^{G}(Z) \rightarrow K_{*}^{G}\left(C_{r}^{*}\left(\Gamma_{0}\right)\right)
$$

Furthermore, let $U$ be the completion of the space of all continuous sections of $V$ with compact support in $Y$, and $\varphi(f)=f I_{U}, f \in C(Y)$. Then $(U, \varphi, 0)$ is a Kasparov $G-C(Y)-\mathcal{A} \otimes C(Y)$-module with $\mathcal{A}=C_{r}^{*}\left(\Gamma_{0}\right)$, and defines an element $\left[\beta_{Y}\right] \in K K^{G}(C(Y), \mathcal{A} \otimes C(Y))$ (cf. [Kas $]$ ). We can consider $\left[\beta_{Z}\right]$ as a restriction of $\left[\beta_{Y}\right]$ to $W \subset Y_{X}$.

We are now going to interpret $\beta$ as a map coming from a Dirac element on $M_{X}$. To this aim, we need a new $G$ - $C^{*}$-algebra $C_{\tau}^{*}(U)$. Let $C^{*}(U)$ be the $G$ - $C^{*}$-algebra of the groupoid $U=\bigcup_{x, y \in X} U_{x, y}$ associated with the covering $\left\{U_{x}, U_{x, y}, I\right\}_{x, y \in X}$ of $M_{X} \cdot C^{*}(U)$ is the completion of $C_{c}(U)=\bigoplus_{x, y \in X} C_{c}\left(U_{x, y}\right)$ with the norm given by $\|f\|=\sup \left\{\left\|f_{s}\right\|, s \in M_{X}\right\}$ for $f=\bigoplus_{x, y} f_{x, y}$ in $C_{c}(U)$, and $\left\|f_{s}\right\|$ is the operator norm on $H_{s}=l^{2}\left(\left\{x \in X ; s \in U_{x}\right\}\right)$, since $f$ defines a finite rank operator $f_{s}$ on $H_{s}$ by $\left\langle e_{x}, f_{s}\left(e_{y}\right)\right\rangle=f(x, y, s)=f_{x, y}(s)$. The product of $C^{*}(U)$ is the convolution given by $\left(f_{1} f_{2}\right)_{x, y}=\sum_{z} f_{1, x, z} f_{2, z, y}$ for $f_{i}=\bigoplus_{x, y} f_{i, x, y}$ in $C_{c}(U)$. As pointed out in [KaS], $C^{*}(U)$ is independent of the covering of $M_{X}$ up to Morita equivalence. Let $C_{\tau}^{*}(U)=C^{*}(U) \hat{\otimes} \operatorname{Cliff}(E)$, where $\operatorname{Cliff}(E)$ is the Clifford algebra associated with the positive quadratic form of $E$; i.e., it is the $C^{*}$-algebra generated by $E$ subject to the relations $\xi=\xi^{*}, \xi^{2}=\|\xi\|^{2}, \xi \in E$.

Lemma $16([\mathrm{KaS}])$. Let $(X, B)$ be a locally finite Euclidean building and $M_{X}$ its associated non-Hausdorff manifold.

(a) Let $p$ be a trivial map from $M_{X}$ to a fixed point. Then $p_{\text {! defines a Gysin }}$ element $p_{!} \in K K^{\Gamma}\left(C_{\tau}^{*}(U), \mathbb{C}\right)$ which is called the Dirac element $D_{X}$.

(b) There is an element $\eta_{X} \in K K^{\Gamma}\left(\mathbb{C}, C_{\tau}^{*}(U)\right)$ such that

$$
D_{X} \otimes_{\mathbb{C}} \eta_{X}=I_{C_{\tau}^{*}(U)} \in K K^{\Gamma}\left(C_{\tau}^{*}(U), C_{\tau}^{*}(U)\right)
$$

With the above preparation, we can prove the following injectivity of the assembly map $\beta$.

Proposition 3. Let $(X, B)$ be a locally finite Euclidean building and $\Gamma_{0}$ a finitely generated discrete group acting on $(X, B)$ properly and freely by type permuting isometries. Suppose $G$ is a compact Lie group acting on $Y=B / \Gamma_{0}$ by isometries with a fixed point. Then $\beta: K_{*}^{G}(Y) \rightarrow K_{*}^{G}\left(C_{r}^{*}\left(\Gamma_{0}\right)\right)$ is injective. 
Proof. As shown by Kasparov ([Kas], Theorems 4.10,6.6 and 6.7), in view of [KaS], we have that the map $\beta$ is the composite

$$
\begin{array}{ccl}
K_{*}^{G}\left(Y_{X}\right) & \begin{array}{c}
\text { Poincaré duality } \\
\simeq
\end{array} & K_{*}^{G}\left(C_{\tau}^{*}\left(U / \Gamma_{0}\right)\right) \\
& \stackrel{\simeq}{\text { Green-Julg Thm. }} & K_{*}\left(C_{\tau}^{*}\left(U / \Gamma_{0}\right) \rtimes G\right) \\
& \stackrel{\text { Morita }}{\simeq} \text { equiv. } & K_{*}\left(C_{\tau}^{*}(U) \rtimes \Gamma\right) \\
& j^{\Gamma}\left[D_{X}\right] & K_{*}\left(C_{r}^{*}(\Gamma)\right) \simeq K_{*}^{G}\left(C_{r}^{*}(\pi(Y)) \simeq K_{*}^{G}\left(C_{r}^{*}\left(\Gamma_{0}\right)\right),\right.
\end{array}
$$

where $\Gamma=\Gamma_{0} \rtimes G$ and $j^{\Gamma}$ is the reduction map ([Kas], Theorem 3.11), $j^{\Gamma}\left[D_{X}\right] \in$ $K K\left(C_{\tau}^{*}(U) \rtimes \Gamma, C_{r}^{*}(\Gamma)\right)$. This is similar to those in [RoW2] for manifolds of nonpositive curvature. The Morita equivalence above follows from the fact that by Theorem 1 of [CMW] and Theorem 3.13 of [Kas],

$$
\begin{aligned}
K_{*}\left(C_{\tau}^{*}\left(U / \Gamma_{0}\right) \rtimes G\right) & \simeq K_{*}\left(C_{\tau}^{*}(U)^{\Gamma_{0}} \rtimes G\right) \\
& \stackrel{\text { Morita equiv. }}{\simeq} K_{*}\left(\left(C_{\tau}^{*}(U) \rtimes \Gamma_{0}\right) \rtimes G\right) \simeq K_{*}\left(C_{\tau}^{*}(U) \rtimes \Gamma\right) .
\end{aligned}
$$

The Poincaré duality follows from Theorem 6.8 of $[\mathrm{KaS}]$ and the fact that, by (9), the non-Hausdorff manifold $Y_{X}=M_{X} / \Gamma_{0}$ and $Y$ are $G$-equivariantly homotopy equivalent. By Lemma $16, j^{\Gamma}\left[D_{X}\right] \otimes_{C_{r}^{*}(\Gamma)} j^{\Gamma}\left[\eta_{X}\right]=I_{C_{\tau}^{*}(U) \rtimes \Gamma}$. We obtain that $\beta$ is injective.

We should point out that the condition on the fixed point of $G$ in Proposition 3 is only to guarantee the Morita equivalence in the above proof. Another extremal case is when $G$ acts freely on $(X, B)$. By the proof of Proposition 3 and Theorem 3.13 of [Kas], the Morita equivalence holds also for this case.

We now prove the homotopy invariance of the higher equivariant signature for groups acting on Euclidean buildings. Note that the action of a locally compact group on a Euclidean building is given by a homomorphism from the group to the isometry group of the building.

Theorem 5. Let $(X, B)$ ba a locally finite Euclidean building, $\Gamma_{0}$ a discrete group acting on $(X, B)$ properly and freely by type permuting isometries. Suppose that the compact Lie group $G$ acts on $Y=B / \Gamma_{0}$ by isometries with a fixed point. Then for every $G$-equivariant commutative diagram $(8), \varphi_{*}\left(\left[D_{M}\right]\right)=\psi_{*}\left(\left[D_{N}\right]\right)$.

Proof. By Proposition 3, we know that $\beta: K_{*}^{G}(Y) \rightarrow K_{*}^{G}\left(C_{r}^{*}\left(\Gamma_{0}\right)\right)$ is injective. It is thus sufficient to show that $\beta\left(\varphi_{*}\left(\left[D_{M}\right]\right)\right)=\beta\left(\psi_{*}\left(\left[D_{N}\right]\right)\right)$. Hence, the problem reduces to the $G$-pseudo-equivalence of the invariant $\beta\left(\varphi_{*}\left(\left[D_{M}\right]\right)\right)$. In view of the construction of $\beta$ above, the $K K$-index theorem [RoW1] shows that

$$
G-\operatorname{Sig}\left(D_{\varphi^{*}(V)}\right)=\beta\left(\varphi_{*}\left(\left[D_{M}\right]\right)\right),
$$

where $V$ is the $G-C_{r}^{*}\left(\Gamma_{0}\right)$-bundle over $Y$ constructed in the definition of $\beta$. Theorem 1 implies that $G$ - $\operatorname{Sig}\left(D_{\varphi^{*}(V)}\right)$ is a $G$-pseudo-equivalence invariant.

Now Theorem 3 follows immediately from Theorem 5 .

Proof of Theorem 3. Take $\Gamma_{0}=\{1\}$. Then $G$ acts on $B$. In view of the Bruhat-Tits fixed-point theorem ([Bro], p.157), $G$ has a fixed point. Hence Theorem 5 is applicable. In fact, when $\Gamma_{0}=\{1\}$, the Morita-equivalence is trivial and Proposition 3 is true without the condition on the fixed point. 
Combining Theorem 1 with the proof above (cf. also [RoW2]), we can remove the finite generation condition on $K_{*}^{G}(Y)$ over $R(G)$ in the Rosenberg-Weinberger theorem. The finite generation condition of $K_{*}^{G}(Y)$ enables one to reduce the compact group action to the finite group action using the McClure theorem.

Theorem 6. Let $Y$ be a complete Riemannian $G$-manifold of nonpositive curvature in the commutative diagram (8). Suppose $G$ has a fixed point in $Y$. Then $\varphi_{*}\left(\left[D_{M}\right]\right)=\psi_{*}\left(\left[D_{N}\right]\right)$ in $K_{*}^{G}(Y)$.

In particular, if $Y$ is a complete simply connected Riemannian $G$-manifold of nonpositive curvature, then by the Cartan theorem (cf. [Bro], p. 157), $G$ has a fixed point in $Y$. Hence the condition on the fixed point in Theorem 6 is satisfied in this case.

\section{Existence of Equivariant Maps}

In this section we will prove Theorem 4, which is about the existence of the equivariant maps in (8). In [KoS] Korevaar and Schoen proved the existence of a $\Gamma_{0}$-equivariant map from $\tilde{M}$ to a metric space $W$ of nonpositive curvature, where $\Gamma_{0}$ is a finitely generated discrete group acting on $\tilde{M}$ and $W$ by isometries (cf. also $[\mathrm{GrS}])$. We want to extend their theorem to the $\Gamma$-equivariant case, where $\Gamma$ is the extension of $\Gamma_{0}$ by the compact Lie group $G$,

$$
1 \rightarrow \Gamma_{0} \rightarrow \Gamma \rightarrow G \rightarrow 1
$$

In our situation, we take $\Gamma_{0}=\pi_{1}(M)$ and

$$
\Gamma=\left\{\beta_{g}=\left(g, \psi_{g}\right) \in G \times \operatorname{Diff}(\tilde{M}): \pi_{M} \psi_{g}=g \pi_{M}\right\},
$$

where $\pi_{M}: \tilde{M} \rightarrow M$ is the natural projection. $\psi_{g}$ is called a lifting of $g \in G$ to $\tilde{M}$. Any two liftings of $g$ differ by an element of $\Gamma_{0} . \Gamma$ is a locally compact group and acts on $\tilde{M}$ via $\psi_{g}$. Let $\rho: \Gamma \rightarrow \operatorname{Isom}(W)$ be a continuous homomorphism, where the isometry group $I \operatorname{som}(W)$ of $W$ is endowed with a compact-open topology. We thus have an action of $\Gamma$ on $W$ via $\rho$.

Recall that a complete metric space $W$ is of nonpositive curvature (cf., for instance, $[\mathrm{KoS}])$ if: 1) $W$ is a length space, i.e., the distance $d(x, y)$ of any two points $x$ and $y$ in $W$ can be realized as the length of a rectifiable curve connecting $x$ to $y$ (those distance-realizing curves are called geodesics); 2) for any three points $x, y, z$ in $W$, let $\nu_{x y}, \nu_{y z}$ and $\nu_{z x}$ be three geodesics connecting these three points, respectively. If $\bar{y}$ is the midpoint on $\nu_{y z}$, i.e., $d(y, \bar{y})=\frac{1}{2} d(y, z)=d(\bar{y}, z)$, then

$$
d^{2}(x, \bar{y}) \leq \frac{1}{2} d^{2}(x, y)+\frac{1}{2} d^{2}(x, z)-\frac{1}{4} d^{2}(y, z) .
$$

A locally compact metric space of nonpositive curvature must be simply connected.

We now want to prove Theorem 4.

Proof of Theorem 4. Step 1. Existence. The proof is to extend the argument for $\Gamma_{0}$-equivariant maps $[\mathrm{KoS}]$ to our case. Let $\tilde{M}_{0}$ be a compact fundamental domain of $\Gamma_{0}$ in $\tilde{M}$ such that the boundary of $\tilde{M}_{0}$ has zero measure. $\tilde{M}$ is a union of all $\gamma \tilde{M}_{0}, \gamma \in \Gamma_{0}$, up to a zero measure set. Fix a point $q_{0} \in W$ and define a map $f$ from $\Gamma$ to $W$ as

$$
f\left(\beta_{g}\right)=\rho\left(\beta_{g}\right) q_{0}
$$


Obviously, $f$ is a $\Gamma$-equivariant map. Let $\mu$ be the Riemannian volume measure on $\tilde{M}$ which is the lifting of a Riemannian volume measure on $M$. For $x \in \tilde{M}$, denote by $B(x, 1)$ the unit ball in $\tilde{M}$ centered at $x$. Define a measure $\mu_{x}$ on $\Gamma$ by setting

$$
d \mu_{x}\left(\beta_{g}\right)=\frac{\mu\left(B(x, 1) \cap \beta_{g}\left(\tilde{M}_{0}\right)\right)}{\mu(B(x, 1))} d \mu_{G}(g),
$$

where $\mu_{G}$ is the normalized Haar measure on $G$. For $g \in G$, let

$$
\Gamma_{g}=\left\{\beta_{g} \in \Gamma: \beta_{g}=\left(g, \psi_{g}\right), \psi_{g} \text { is a lifting of } g \text { to } \tilde{M}\right\} .
$$

Then $\Gamma_{g} \simeq \Gamma_{0}$. Using the fact that, for a fixed $g \in G$,

$$
\mu\left(B(x, 1) \cap \bigcup_{\beta_{g} \in \Gamma_{g}} \beta_{g}\left(\tilde{M}_{0}\right)\right)=\mu(B(x, 1)),
$$

we have that $d \mu_{x}$ is a probability measure on $\Gamma$. Furthermore, one can easily check that $d \mu_{x}$ is $\Gamma$-equivariant, i.e., $d \mu_{\beta_{g^{\prime}}(x)}\left(\beta_{g^{\prime}} \beta_{g}\right)=d \mu_{x}\left(\beta_{g}\right)$. Consider the integral

$$
I_{x}(q)=\int_{\Gamma} d^{2}\left(f\left(\beta_{g}\right), q\right) d \mu_{x}\left(\beta_{g}\right), \quad q \in W,
$$

where $d\left(f\left(\beta_{g}\right), q\right)$ is the distance between $f\left(\beta_{g}\right)$ and $q$ in $W$. Clearly, this integral is well defined (see (10), below). By Lemma 2.5.1 of [KoS], there exists a unique point $\bar{f}_{x}$ in $W$, called the center of mass of $f$, that minimizes the integral $I_{x}(q)$. We claim that $\bar{f}_{x}$ is $\Gamma$-equivariant in $x$, namely, $\bar{f}_{\beta_{g}(x)}=\rho\left(\beta_{g}\right)\left(\bar{f}_{x}\right)$. Indeed, by the $\Gamma$-equivariance of $d \mu_{x}$, we have that $I_{\beta_{g}(x)}(q)=I_{x}\left(\beta_{g}^{-1}(q)\right)$. The uniqueness of the center of mass implies the claim.

We define a map $u: \tilde{M} \rightarrow W$ by $u(x)=\bar{f}_{x} . u$ is $\Gamma$-equivariant.

Step 2. Continuity. Let $x \in M$ and $\Gamma_{x}=\left\{\beta_{g} \in \Gamma: \mu\left(\beta_{g}\left(\tilde{M}_{0}\right) \cap B(x, 1)\right) \neq 0\right\}$. By the $\Gamma$-equivariance of $u$, it is sufficient to check the continuity of $u$ at $x$ in an $\varepsilon$ neighborhood of $\tilde{M}_{0}(\varepsilon>0$ very small). We first prove that there exists a constant $k>0$ such that for all $\beta_{g} \in \Gamma_{x}$,

$$
d\left(f\left(\beta_{g}\right), q_{0}\right)<k .
$$

Indeed, let $g \in G, \beta_{g}=\left(g, \psi_{g}\right) \in \Gamma_{x}$, and $\left\{\gamma_{1}, \cdots, \gamma_{r}\right\}$ be a finite set of generators of $\Gamma_{0}$. Then there is a constant $k_{g}>0$ such that for every lifting $\psi_{g}^{\prime}$ of $g$ with $\beta_{g}^{\prime}=\left(g, \psi_{g}^{\prime}\right) \in \Gamma_{x}, \psi_{g}^{\prime}$ differs from $\psi_{g}$ only by a $\gamma \in \Gamma_{0}$ and the word length $|\gamma|$ of $\gamma$ with respect to the above finite set of generators is less than $k_{g}$. By the covering property of the projection $\Gamma \rightarrow \Gamma / \Gamma_{0} \simeq G$ and the continuity of $\rho$, for any $g^{\prime} \in G$ very close to $g$, we can choose a lifting $\psi_{g^{\prime}}$ of $g^{\prime}$ to $\tilde{M}, \beta_{g^{\prime}}=\left(g^{\prime}, \psi_{g^{\prime}}\right) \in \Gamma_{x}$, such that $d\left(\beta_{g^{\prime}}\left(\tilde{M}_{0}\right), \beta_{g}\left(\tilde{M}_{0}\right)\right)<\varepsilon_{g}$ for some constant $\varepsilon_{g}>0$. Thus for any other lifting $\psi_{g^{\prime}}^{\prime}$ of $g^{\prime}, \beta_{g^{\prime}}^{\prime}=\left(g^{\prime}, \psi_{g^{\prime}}^{\prime}\right) \in \Gamma_{x}, \psi_{g^{\prime}}^{\prime}$ differs from $\psi_{g^{\prime}}$ by a $\gamma^{\prime} \in \Gamma_{0}$ with $\left|\gamma^{\prime}\right|<k_{g}$. This is true for $g^{\prime} \in U_{g}$, where $U_{g}$ is a small neighborhood of $g$ in $G$. We have

$$
\begin{aligned}
d\left(f\left(\beta_{g^{\prime}}^{\prime}\right), q_{0}\right) & =d\left(\rho\left(\beta_{g^{\prime}}\right) \gamma^{\prime} q_{0}, q_{0}\right) \\
& \leq d\left(q_{0}, \rho\left(\beta_{g^{\prime}}\right)^{-1} q_{0}\right)+d\left(\gamma^{\prime} q_{0}, q_{0}\right) \\
& \leq d\left(f\left(\beta_{g^{\prime}}\right), q_{0}\right)+k_{g} \max _{1 \leq j \leq r} d\left(\gamma_{j} q_{0}, q_{0}\right) \\
& \leq c_{g} d\left(f\left(\beta_{g}\right), q_{0}\right)+k_{g} \max _{1 \leq j \leq r} d\left(\gamma_{j} q_{0}, q_{0}\right),
\end{aligned}
$$

for some constant $c_{g}>0$ depending only on $g \in G$. Here we have used the fact that $\rho\left(\beta_{g^{\prime}}\right)$ is an isometry on $W$. Since $G$ is compact, we can use a finite number of $U_{g}^{\prime} \mathrm{s}$ to cover $G$. This proves (10). Hence, it follows that for $\beta_{g} \in \Gamma_{x}, f\left(\beta_{g}\right)$ 
lies in a convex ball $B\left(q_{0}, k\right) \subset W$. Proposition 2.5.4 of $[\mathrm{KoS}]$ then shows that $u(x) \in B\left(q_{0}, k\right)$. We get

$$
d\left(u(x), f\left(\beta_{g}\right)\right) \leq 2 k
$$

Now for $y \in \tilde{M}$ very close to $x$, we get by a direct computation that

$$
\begin{aligned}
\int_{\Gamma}\left|d \mu_{x}(\gamma)-d \mu_{y}(\gamma)\right| \\
\quad=\int_{G} \int_{\Gamma_{g}}\left|\frac{\mu\left(B(x, 1) \cap \beta_{g}\left(\tilde{M}_{0}\right)\right)}{\mu(B(x, 1))}-\frac{\mu\left(B(y, 1) \cap \beta_{g}\left(\tilde{M}_{0}\right)\right)}{\mu(B(y, 1))}\right| d \mu_{G}(g) \\
\quad \leq c_{x}|x-y|,
\end{aligned}
$$

for some constant $c_{x}>0$ depending only on $x$. This together with (11) and Proposition 2.5.2 of $[\mathrm{KoS}]$ proves that

$$
\begin{aligned}
d^{2}(u(x), u(y)) & \leq 2 d(u(x), u(y)) \int_{\Gamma} d(f(\gamma), u(x))\left|d \mu_{x}(\gamma)-d \mu_{y}(\gamma)\right| \\
& \leq 4 k c_{x} d(u(x), u(y))|x-y| .
\end{aligned}
$$

Hence, $u$ is continuous.

Let $\Gamma_{0}^{\prime}=\rho\left(\Gamma_{0}\right)$ be discrete. Take $Y=W / \Gamma_{0}^{\prime}$ in (8). Then $G$ acts on $Y$ via the homomorphism $\rho$. By pushing down the $\Gamma$-equivariant map in Theorem 4, we get

Corollary 1. With the condition of Theorem 4, assume further that $\Gamma_{0}^{\prime}=\rho\left(\Gamma_{0}\right)$ is discrete. If $Y=W / \Gamma_{0}^{\prime}$, then there exists a $G$-equivariant map $\varphi$ from $M$ to $Y$.

Corollary 1 is applicable to the case where $W$ is the geometric realization of a Euclidean building. Another special case of Theorem 4 is when $M$ is simply connected. Then $\Gamma=G$ acts on $W$ via $\rho$. We conclude that there is a $G$-map from $M$ to $W$ in this case. See also [RoW2] for other special cases of Theorem 4.

Finally, we want to draw a conclusion on the equivariant Novikov conjecture of Baum-Connes-Higson [BCH]. Let $H$ be a Lie group and $W$ an oriented Riemannian manifold with a smooth, proper orientation-preserving $H$-action such that $W / H$ is compact. Here the properness is in the sense of $[\mathrm{BCH}]$. There is a universal classifying space $\underline{E H}$ for the spaces with proper $H$-actions. Thus, we have an $H$-map from $W$ to $\underline{E H}$. The equivariant Novikov conjecture of $[\mathrm{BCH}]$ is that the $H$-equivariant signature $H$-Sig $(W)$ of $W$ is an $H$-pseudo-equivalence invariant after injecting into $K_{*}^{H}(\underline{E H})$. In the situation of Theorem 5, we have a $G$-map from $Y$ to $\underline{E G}=\{p t\}$. Taking $H=G$ and $W=M$, we get

Corollary 2. With the condition of Theorem 5, the equivariant Novikov conjecture of $[\mathrm{BCH}]$ is true, namely, $G$-Sig $(M)$ is a $G$-pseudo-equivalence invariant in $K_{*}^{G}(p t)$.

The argument of the present paper could be used to consider the above conjecture for more general cases.

\section{REFERENCES}

[AtS] M. F. Atiyah and I. Singer, Index theorem of elliptic operators, I, III, Ann. Math., 87 (1968), 484-530, 546-609. MR 38:5243, MR 38:5245

[BaJ] S. Baaj and P. Julg, Théorie bivariante de Kasparov et opérateurs non bornés dans les $C^{*}$-modules hilbertiens, C. R. Acad. Sci. Paris Ser. I, 296 (1983), 875-878. MR 84m:46091 
[BCH] P. Baum, A. Connes and N. Higson, Classifying space for proper action and $K$-theory of group $C^{*}$-algebras, $C^{*}$-Algebras: 1943-1993, A Fifty Year Celebration, R. S. Doran, ed., Contemp. Math., Vol. 167, AMS., Providence, 1994, 241-291. MR 96c:46070

[Bla] B. Blackadar, K-Theory for Operator Algebras, MSRI Publ. Vol. 5, Springer, NY, 1986. MR 88g:46082

[BHM] M. Bökstedt, W. C. Hsiang and I. Madsen, The cyclotomic trace and algebraic $K-$ theory of spaces, Invent. Math., 111 (1993), 465-539. MR 94g:55011

[Bre] G. E. Bredon: Introduction to compact transformation groups, Academic Press, NY, 1980. MR 54:1265

[Bro] K. S. Brown, Buildings, Springer, NY, 1989. MR 90e:20001

[BuS] K. Burns and R. Spatzier, Manifolds of nonpositive curvature and their buildings, Publ. Math. IHES, No. 65, 35-59, 1987. MR 88g:53050

[Cap] S. E. Cappell, On homotopy invariance of higher signatures, Invent. Math., 33 (1976), 171-179. MR 55:13264

[CaP] G. Carlsson and E.K. Pedersen, Controlled algebra and the Novikov conjectures for $K$ and $L$-theory, Topology, 34 (1995), 731-758. MR 96f:19006

[CoJ] R. L. Cohen and J. D. S. Jones, Algebraic $K$-theory of spaces and the Novikov conjecture, Topology, 29 (1990), 317-344. MR 91i:19001

[CGM] A. Connes, M. Gromov and H. Moscovici, Group cohomology with Lipschitz control and higher signature, Geom. Funct. Anal., 3 (1993), 1-78. MR 93m:19011

[CoM] A. Connes and H. Moscovici, Cyclic cohomology, the Novikov conjecture and hyperbolic groups, Topology, 29 (1990), 345-388. MR 92a:58137

[CMW] R. Curto, P. Muhly and D. Williams, Cross products of strongly Morita equivalent $C^{*}$-algebras, Proc. Amer. Math. Soc., 90 (1984), 528-530. MR 85i:46083

[FaJ] F. T. Farrell and L. E. Jones, Rigidity in geometry and topology, Proc. ICM, Kyoto 1990, Springer, 1991, 653-663. MR 93g:57041

[FeW] S. C. Ferry and S. Weinberger, Curvature, tagentiality and controlled topology, Invent. Math., 105 (1991), 401-414. MR 94c:57043

[Gong1] D. Gong, Bivariant twisted cyclic theory and spectral sequences of crossed products, J. Pure. Appl. Alg., 79 (1992), 225-254. MR 93f:16013

[Gong2] D. Gong, Excision of equivariant cyclic cohomology of topological algebras, Michigan Math. J. 39 (1992), 455-473. MR 94f:19005

[Gong3] D. Gong, $L^{2}$-Analytic Torsions, Equivariant Cyclic Cohomology and the Novikov Conjecture, thesis, SUNY at Stony Brook, 1992.

[Gong4] D. Gong, The Chern characters in equivariant cyclic theory, preprint, 1994.

[Gong5] D. Gong, A relation between the Novikov and Thurston conjectures, Applied Functional Anal., Vol. 2, Sci-Tech Information Services, Hong Kong, 1995, 52-62.

[GoR] D. Gong and M. Rothenberg, Analytic torsion forms for noncompact fiber bundles, preprint, 1995.

[GrS] M. Gromov and R. Schoen, Harmonic maps into singular spaces and $p$-adic superrigidity for lattices in groups of rank one, Publ. Math. IHES, No.76, 1993, 165-246. MR 94e:58032

[Hil] M. Hilsum, Fonctorialité en $k$-theórie bivariante pour les variétés lipschitziennes, $K$ Theory, 3 (1989), 401-440. MR 91j:19012

[HiS] M. Hilsum and G. Skandalis, Invariance par homotopie de la signature a coefficients dans un fibré presque plat, J. reine angew. Math., 423 (1992), 73-99. MR 93b:46137

[Hus] D. Husemoller, Fibre Bundles, McGraw-Hill, NY, 1966. MR 37:4821

$[\mathrm{KaM}] \quad$ J. Kaminker and J. G. Miller, Homotopy invariance of the analytic signature operators over $C^{*}$-algebras, J. Oper. Theory, 14 (1985), 113-127. MR 87b:58082

[Kas] G. G. Kasparov, Equivariant $K K$-theory and the Novikov conjecture, Invent. Math., 91 (1988), 147-201. MR 88j:58123

[KaS] G. G. Kasparov and G. Skandalis, Groups acting on buildings, operator $K$-theory and Novikov's conjecture, $K$-theory, 4 (1991), 303-338. MR 92h:19009

[KoS] N.J. Korevaar and R. M. Schoen, Sobolev spaces and harmonic maps for metric space targets, Commun. Anal. Geom., 1 (1993), 1-99. MR 95b:58043

[Lus] G. Lusztig, Novikov's higher signature and families of elliptic operators, J. Diff. Geom., 7 (1971), 229-256. MR 48:1250 
[Mis1] A. S. Mishchenko, Homotopy invariants of non-simply connected manifolds I, Math. USSR Izv. 4 (1970), 506-519. MR 42:3795

[Mis2] A. S. Mishchenko, Infinite dimensional representations of discrete groups, and higher signatures, Math. USSR Izv., 8 (1974), 85-111. MR 50:14848

[Roe] J. Roe, Hyperbolic metric spaces and the exotic cohomology Novikov conjecture, $K$ Theory, 4 (1991), 501-512; 5 (1991), 189. MR 93e:58180a, b

[Ros] J. Rosenberg, $C^{*}$-algebras, positive scalar curvature, and the Novikov conjecture, Publ. Math. IHES, No. 58, 1983, 409-424. MR 85g:58083

[RoW1] J. Rosenberg and S. Weinberger, Higher $G$-indices and applications, Ann. Sci. École Norm. Sup., 21 (1988), 479-495. MR 90f:58170

[RoW2] J. Rosenberg and S. Weinberger, An equivariant Novikov conjecture, $K$-theory, 4 (1990), 28-53. MR 91k:57039

[Ska] G. Skandalis, Approche de la conjecture de Novikov par la cohomologie cyclique, Astérisque, 201-203 (1991), 299-320. MR 93i:57035

[Tits] J. Tits, Buildings of Spherical Type and Finite BN-Pairs, Lecture Notes in Math., No. 386, Springer, NY, 1974. MR 57:9866

[Wein] S. Weinberger, The Topological Classification of Stratified Spaces, Univ. of Chicago Press, Chicago, 1994. MR 96b:57024

Department of Mathematics, University of Chicago, 5734 S. University Ave., Chicago, ILLINOIS 60637

E-mail address: donggeng@math.uchicago.edu 